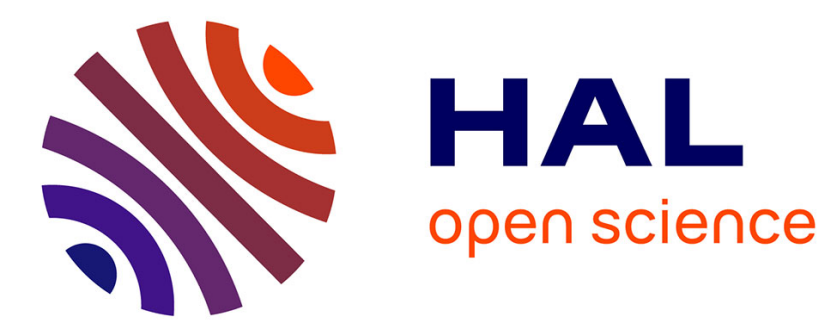

\title{
Mathematical homogenization of inelastic dissipative materials: a survey and recent progress
}

Nicolas Charalambakis, Georges Chatzigeorgiou, Yves Chemisky, Fodil

Meraghni

\section{- To cite this version:}

Nicolas Charalambakis, Georges Chatzigeorgiou, Yves Chemisky, Fodil Meraghni. Mathematical homogenization of inelastic dissipative materials: a survey and recent progress. Continuum Mechanics and Thermodynamics, 2017, 30 (1), pp.1-51. 10.1007/s00161-017-0587-5 . hal-02390365

\section{HAL Id: hal-02390365 \\ https://hal.science/hal-02390365}

Submitted on 3 Dec 2019

HAL is a multi-disciplinary open access archive for the deposit and dissemination of scientific research documents, whether they are published or not. The documents may come from teaching and research institutions in France or abroad, or from public or private research centers.
L'archive ouverte pluridisciplinaire HAL, est destinée au dépôt et à la diffusion de documents scientifiques de niveau recherche, publiés ou non, émanant des établissements d'enseignement et de recherche français ou étrangers, des laboratoires publics ou privés. 


\title{
REVIEW ARTICLE
}

\author{
Nicolas Charalambakis \\ Yves Chemisky • Fodil Meraghni
}

\section{Mathematical homogenization of inelastic dissipative materials: a survey and recent progress}

\begin{abstract}
In this paper, a review of papers on mathematical homogenization of dissipative composites under small strains and on the interplay between homogenization procedure and dissipation due to mechanical work is presented. Moreover, a critical survey on the links between mathematical homogenization and computational homogenization is attempted.
\end{abstract}

Keywords Homogenization · Inelastic composites · Dissipation

\section{Introduction}

The present paper is motivated by the abundant literature on the role of dissipation in mathematical homogenization of dissipative materials, which is crucial: It needs to be considered in both micro- and macrolevel in order to lead to the correct constitutive evolution equations relating stress and internal variables. Contrarily to the elastic materials, in which dissipation is absent, viscoelastic, elastoplastic, and viscoplastic materials are all dissipative. In viscoelasticity, the dissipation potential is a quadratic function of the strain rate $\dot{\varepsilon}$, and one obtains a dissipation inequality of the form $\gamma_{\text {loc }}=\dot{\varepsilon}: V: \dot{\varepsilon} \geq 0$, where $V$ is the viscoelastic fourth-order symmetric tensor. In elastoplasticity, the (singular) dissipation potential is defined as the support function of the elasticity convex $K$, which is the Legendre-transformed of the indicator function, and the dissipation inequality is given by the expression $\gamma_{\mathrm{loc}}=\sigma: \dot{\boldsymbol{\varepsilon}}^{\mathrm{p}}+q \dot{\xi} \geq 0$, where $\varepsilon^{\mathrm{p}}, \sigma, \xi$ and $q$ are the plastic strain tensor, the stress tensor, the scalar internal variable related with the plastic mechanism and the thermodynamic force connected with the $\xi$, respectively. In viscoplasticity, the dissipation potential is obtained by regularization, 
for instance via Yosida transformation. For a study of the dissipative behavior, the reader is referred to Gurtin et al. [77] and Babadjian [18].

On the basis of the fundamental works of Halphen and Nguyen [79], Germain [74], and Nguyen [157], the local problem in generalized standard materials (GSM) is completely described by state and internal variables. Generalized forces are then defined from the free energy function expression in terms of the above variables. Additionally, the dissipation inequality holds and by the Lagrange multiplier's technique gives the evolutionary equations.

In this introductory part, it is first recalled the following important preliminaries from elasticity theory: The existence of solutions of the incremental boundary value problems is based on (i) the so-called initial stress problem, which consists of a boundary value problem with initial stress by prescribing additional incremental displacements on a part of the boundary and additional tractions on the remainder boundary, and of the related existence of a strain energy function $\mathrm{d} w=\sigma: \mathrm{d} \boldsymbol{\varepsilon}$ and (ii) the existence theory of linearized elasticity. It is reminded that, in incremental plasticity, strain-hardening materials are characterized by the existence of such an energy function [86], and consequently two variational principles can be created for the kinematically admissible and statically admissible solutions. Concerning the elasticity problem, Ciarlet defined the general functional setting for the existence theory using Lax-Milgram theorem and Korn's inequality [45]. The same author defined the functional setting for the weak form of momentum equation in initial boundary value problems involving the velocity field, regardless of any constitutive assumption.

Material heterogeneities, possibly combined with strain and/or temperature initial non-uniformities, may cause heterogeneity-induced inelastic phenomena, such as adiabatic shear banding or necking, as well as non-uniqueness cases, unlike those observed in strictly homogeneous materials. Consequently, the possible discontinuities of strain and/or displacement must be captured by the appropriate functional setting for the solutions of the partial differential equations describing the problem and a generic existence and uniqueness related theory is of great importance. Based on the seminal work of Hill [86], a rigorous theory for rateindependent plasticity was presented by Duvaut and Lions [58], where the authors proved the existence and uniqueness of generalized stress and velocity fields of the initial boundary value problem of elastoplasticity based on the dissipation inequality written in terms of generalized variables [180]. The dissipativity thus provides an a priori stability estimate for the solutions. Moreover, the total dissipation is equal to the difference between total internal power and total internal free power, being always nonnegative. From Johnson [100], where a mixed finite element method for plasticity with hardening was presented, to Simo and Hughes [180], where a complete integration algorithm was proposed for the iterative solution of a discretized version of the momentum equations, computational homogenization in elastoplasticity appears to be an efficient method, provided that it is based on the correct functional setting, depending on the material response. Of special interest is the strain-driven procedure [180], where the state variables are computed for a given deformation path. The stress tensor is viewed as a dependent variable, defined in terms of the elastic part of strain by means of the gradient of the stored energy function, while the elastic part of energy is computed (i) only at quadrature points and (ii) for prescribed total strain.

Concerning the interplay between mathematical homogenization and finite element methods (FEM), the latter need to combine with the former, since the heterogeneities govern the size of elements resulting to very expensive discretization, and questions as "distance" between functions, approximating a function and its gradient or limits of oscillating quantities need to be answered. Existence and uniqueness theorems and related functional setting determine the efficiency of numerical methods. The ideal functional setting for FEM is the space of $L^{2}$-strain and stress and $H^{1}$-displacement $[8,70,212]$. The same functional setting is suitable in the one-dimensional thermoviscoplasticity [33], but this is not the case of materials without hardening, where the space of functions with bounded deformation ( $B D$-displacement, $[123,185,186])$ is needed to describe the development of strain discontinuities, increasing the difficulty of numerical solution. Distributional derivatives and weak convergence are indispensable in order to describe the homogenization problems. However, another topology is needed for the convergence of products of two weakly convergent functions, as the stress in elasticity, which is the product of elasticity tensor and strain, or the internal power, which is the product of stress and strain: the definition of $H$-convergence and the compensated compactness lemma $[148,151,196-$ 198] form the basis of homogenization in linear and nonlinear elasticity, following the fundamental work on $G$-convergence $[46,48,49,52,184,201]$. Another weak convergence, the two-scale convergence, gives a more detailed limit for the stress and strain of periodic microstructured material independently of constitutive assumptions $[4,118,156]$. Thanks to the two-scale convergence of derivatives of $H^{1}$-functions, the existence of microdiplacement is confirmed. A generalization of the two-scale convergence to the case of multiple separated scales of periodic oscillations is proposed by Allaire and Briane [7]. Homogenization of initial/boundary 
problems needs first that the problem is well posed and then that one solution at least exists together with an a priori estimate in some functional space. Passing to the limit, as the size of heterogeneities $\epsilon$ tends to zero, is not easy: One can pass to the limit in the linear equations (equilibrium equation, compatibility equations, initial and boundary conditions (if any)), but one cannot pass to the limit in the nonlinear equations, as the constitutive equation (in nonlinear problems), the energy equation, the dissipation inequality, and the yield function.

Except from the mathematical approach to the homogenization problem, there is a vast amount of literature on the micromechanics techniques based on more engineering approaches. The pioneering works of Eshelby [63], Hill [89], Hill and Rice [92] and Hashin [82] provided the appropriate background for the exploitation of these methods. The famous mean field theories $[147,154,174]$ based on the Eshelby's inclusion and inhomogeneity problems have been an extremely valuable tool toward the estimation of the average linear composites response when the microstructure consists of a matrix phase and various, at most ellipsoidal, inhomogeneities (i.e., particles or fibers) in random distribution, aligned or misaligned inside the matrix. The extensions proposed by several authors, with most famous the Mori-Tanaka technique [146], allow to treat composites with moderate or even high volume fraction of inhomogeneities, while the self-consistent method permits the study of composites or polycrystalline materials where there is no distinct matrix phase. Certain propositions exist in the literature for extending the mean field methods applicability to nonlinear composites $[31,53,55,91,96,111,125,127,128]$. These propositions are not always successful, especially when the matrix phase has nonlinear response, and certain engineering motivated improvements are required [31]. Apart from the mean field methods, there are also special analytical techniques for studying particulate and unidirectional fiber composites [41,44,83], but they are generally limited in terms of phases anisotropy and inelastic response.

Of special technological interest is the mathematical modeling of composites materials [43], for instance optimization problems in elasticity by the homogenization method, such as explicit optimal bounds of effective properties [10,73], shape or topology optimization $[5,9,11]$, relaxed formulation allowing for microperforated composites as admissible designs for minimizing the sum of elastic compliance and weight of a structure under specific loading [6], introducing new numerical algorithm with penalization technique which allows to remove composite designs in the final shape (topology algorithm, since independent of number of holes). Concerning the effective parameters in nonlinear problems, their explicit formulas are very rare and they depend on the boundary conditions and body forces, as well as on the initial strain and temperature. The above asymptotic homogenization must be accompanied by an error estimate, which can be improved in multiscale FEM by appropriate change of the basis functions. A macro-microapproach is then based on a coarse mesh for the nodal values and a fine mesh for computing independently an adapted FE basis for the coarse mesh [8].

Most of the composites used nowadays in structural applications present strong material nonlinearities: From metal-metal and metal-ceramic composites (showing elastoplastic behavior) to polymeric-reinforced materials (presenting nonlinear viscous effects), a proper homogenization study cannot neglect the strong inelastic mechanisms appearing in the microstructural level. In this review, different inelastic models will be presented, and the corresponding homogenization results will be discussed. Dissipation potential-based methods, in which the evolution equations can be derived from this potential, are presented. Then incremental variational principles for time-discretized evolution equations can be used at both micro- and macrolevel. In the history of existence theory of rate-independent plasticity, the first works consider this problem as the limit of hardening or viscoplastic problems, when the hardening parameter or viscosity, respectively, tends to zero. It seems that, in the case of rate-independent plasticity, the macroscopic thermodynamic picture is in contradiction with the microscopic one $[23,103,172]$ : The microscopic physical cutoffs, with zero and extremely large strain rates mixed, justify the singular macroscopic dissipative potential at zero strain rates [172]. Based on the fact that, during quasistatic plastic deformation, the experiments show that the dislocation dynamics is intermittent in space and time with rest periods interrupted by energetic activities with instantaneous strain rate much larger than the imposed one, Puglisi and Truskinovsky [172] consider that rate independence is the homogenized result of rate dependence with respect to both small times and small lengths.

An important difference between homogenization of problems with linear constitutive laws and homogenization of problems with nonlinear constitutive laws, is the loss of stability by homogenization appearing in the nonlinear case $[35-37,188,193,197]$. In addition, while effective elastic properties are independent of the boundary conditions, in plasticity the effective thermomechanical properties crucially depend on the type and the value of prescribed boundary data. Considering nonlinear materials, of special interest are the memory effects arising in rate-independent problems with time-dependent boundary conditions, or thermoelasticity problems, or rate-dependent plasticity $[70,198,200]$. Recently, the homogenization in infinitesimal dislocationbased gradient viscoplasticity with linear kinematic hardening and non-associative monotone plastic flow has 
been studied [155], with a proof starting from a generalized version of Korn's inequality for non-symmetric stress tensors (plastic distortion [153]). These homogenization features are not under the scope of the paper.

For an easier reading, it is recalled that:

(i) Elastic, elastic-plastic with hardening, and elastic-viscoplastic materials have $H^{1}$-displacements and $L^{2}$ stress and strain. Elastic-plastic materials with hardening are characterized by a smooth in time evolution. For a fixed time interval, the Sobolev space fits ideally, without discontinuities, without safe loading control, and with a complete computational scheme (return mapping algorithm [180]).

(ii) Elastic-perfectly plastic materials exhibit a "pathological" dissipation and a smooth in time evolution, but they need a save loading control. The displacement belongs to the space of bounded deformation, the strain is a measure, and the evolution may lead to discontinuities of displacement, strain localization, and shear banding failure [54,218].

Finally, we should stress the fact that the finite strain plasticity is out of the scope of this work.

The paper is organized as follows: After this introductory section, the second section is devoted to the most important notations and definitions related to mechanics and mathematics. In Sect. 3, a review on the existence and uniqueness theorems of plasticity is presented, including the appropriate functional setting for elastic-perfectly plastic, elastic-viscoplastic, and hardening materials, as well as the kinematical framework and related dissipation issues. Section 4 deals with classical homogenization of rate-independent materials. Section 5 presents the role of dissipation inequality in generalized standard materials. In Sect. 6, the most important incremental variational homogenization results for viscous materials are presented, including twopotential variational technique for generalized standard materials. In Sect. 7, a parenthesis with a discretized variational framework is opened, with Hu-Washizu-Simo-Hughes variational problem. In Sect. 8, the exact mathematical homogenization theory of the elastoplastic model is presented, including recent progress in heterogeneous perfect plasticity, slip lines, and criteria for shear banding.

\section{Notations and preliminary definitions}

In this paper, frequent use of the indicial notation and the Einstein summation convention appears.

\subsection{General notations}

$\Re^{3}$ is the three-dimensional Euclidean space.

$I_{A}=\left\{\begin{array}{ll}1 & x \in A \subseteq \mathfrak{R}^{3} \\ 0 \text { otherwise }\end{array}\right.$ is the characteristic function.

$\Im_{A}=\left\{\begin{array}{ll}0 & x \in A \subseteq \Re^{3} \\ +\infty & \text { otherwise }\end{array}\right.$ denotes the indicator function.

$M_{\mathrm{sym}}^{3 \times 3}$ is the space of all $3 \times 3$ symmetric matrices with scalar product $(\boldsymbol{\sigma}, \boldsymbol{\varepsilon}) \equiv \sigma_{i j} \varepsilon_{i j}$.

The product of a matrix $\boldsymbol{A}$ and a vector $\boldsymbol{b}$ is denoted by $[\boldsymbol{A} \cdot \boldsymbol{b}]_{i}=A_{i j} b_{j}$.

$\boldsymbol{a} \cdot \boldsymbol{b}=a_{i} b_{i}$ is the scalar product of two vectors.

$D_{\text {sym }}^{3 \times 3}$ is the space of all $3 \times 3$ symmetric deviatoric matrices.

$\boldsymbol{a} \odot \boldsymbol{b}$ denotes the symmetric tensor product, $[\boldsymbol{a} \odot \boldsymbol{b}]_{i j}=\frac{1}{2}\left[a_{i} b_{j}+b_{i} a_{j}\right]$.

$A C$ is the collection of all absolutely continuous on $I$ functions $f$ (having derivative $f^{\prime}$ almost everywhere, the derivative being Lebesgue integrable and satisfying $\left.f(t)=f(a)+\int_{a}^{t} f^{\prime}(s) d s\right)$.

$\frac{\partial}{\partial \boldsymbol{x}}$ denotes the distributional differentiation with respect to $\boldsymbol{x}$.

$\nabla f(\boldsymbol{x})$ or $\nabla_{\boldsymbol{x}} f$ denotes the distributional gradient with respect to $\boldsymbol{x}$.

$L^{p}\left(\Omega ; \Re^{m}\right)$ is the Lebesgue space of all functions $f(\boldsymbol{x})$ from $\Omega$ to $\Re^{m}$ with finite norm $\left[\int_{\Omega}|f(\boldsymbol{x})|^{p} \mathrm{~d} \boldsymbol{x}\right]^{\frac{1}{p}}$.

(., .) denotes the $L^{2}$ inner product in $\Omega$.

$W^{l, p}\left(\Omega ; \Re^{m}\right)$ is the Sobolev space of all functions $f(\boldsymbol{x})$ from $\Omega$ to $\Re^{m}$ with finite norm $\left[\int_{\Omega} \sum_{\alpha=0}^{l} \mid \nabla^{\alpha}\right.$ $\left.\left.f(\boldsymbol{x})\right|^{p} \mathrm{~d} \boldsymbol{x}\right]^{\frac{1}{p}}$. 
$H^{1}\left(\Omega ; \Re^{m}\right)=W^{1,2}\left(\Omega ; \Re^{m}\right)$ denotes the Sobolev space of all functions $f(\boldsymbol{x})$ from $\Omega$ to $\Re^{m}$ with finite $\operatorname{norm}\left[\int_{\Omega} \sum_{\alpha=0}^{1}\left|\nabla^{\alpha} f(\boldsymbol{x})\right|^{2} \mathrm{~d} \boldsymbol{x}\right]^{\frac{1}{2}}$.

$\mathfrak{B}$ denotes the Borel $\sigma$-algebra of a topological space $X$.

\subsection{Measures}

$(X, \mathfrak{S})$ is a measurable space.

$\mu: \mathfrak{B} \mapsto[0, \infty]$ is Borel measure on $\Re$, for which $\mu(\emptyset)=0$ and

$$
\mu\left(\bigcup_{n \in N} E_{n}\right)=\sum_{n \in N} \mu\left(E_{n}\right)
$$

for any countable collection of disjoint sets $E_{n}$.

$\mathfrak{L}: \mathfrak{B}[0,+\infty]$ denotes Lebesgue measure.

$\mu: \mathfrak{B} \mapsto[0,+\infty)$ denotes finite measure.

$\mu, v: \mathfrak{B} \mapsto[0,+\infty), \mu \perp v: \exists E \in \mathfrak{B}$ such that $\mu(E)=0$ and $v\left(E^{c}\right)=0$ mutual singular or $\mu$ singular with respect to $v$ or $v$ singular with respect to $\mu$.

$|\mu|$ is the total variation.

$v \ll \mu: v: \mathfrak{B} \mapsto[-\infty,+\infty]$ is absolutely continuous with respect to $\mu: \mathfrak{B} \mapsto[0,+\infty]$. If $\mu(E)=0$, then $v(E)=0$ for any $E \in \mathfrak{B}(\nu \ll \mu \Rightarrow|\mu| \ll v)$.

$M_{1}\left(\Omega ; \Re^{m}\right)$ is the space of all bounded Radon measures on $\Omega$ with values in $\Re^{m}$.

$\xi$ is Radon measure: Borel measure that is finite on compact sets.

$\xi$ Radon measure $\Rightarrow \phi \mapsto \int \phi \mathrm{d} \xi$ positive linear functional on the space of $\phi$ continuous with compact support.

$M_{1}^{+}(E)$ is the space of positive bounded Radon measures on $E$.

$\mathfrak{L}^{3}$ is the Lebesgue measure on $\mathfrak{R}^{3}$.

$\mathfrak{H}^{2}$ denotes the two-dimensional Hausdorf measure.

$\mu \in M_{1}^{+}\left(\Re^{3}\right), \mu^{s} \perp \mathfrak{L}^{3}, \mu^{a} \ll \mathfrak{L}^{3}: \mu^{s}+\mu^{a}$ denotes the Radon-Nikodym decomposition.

For a measure $v$ defined on $(X, \mathfrak{M})$ and $v \ll \mu, f=\frac{\mathrm{d} \mu}{\mathrm{d} \nu}$ denotes the Radon-Nikodym derivative.

$\frac{\mathrm{d} v}{\mathrm{~d}|v|}$ is the Radon-Nikodym derivative with respect to the total variation.

$\eta \in M_{1}^{+}(E), x \mapsto \mu_{x} \in M_{1}(F)$ is $\eta$-measurable if $x \mapsto \mu_{x}(B)$ is measurable for every Borel set $B \subseteq F$.

If $x \mapsto\left|\mu_{x}\right|(F)$ is $\eta$-summable and $f \in C_{0}^{0}(E \times F)$, then $\eta \otimes^{\text {gen }} \mu_{x} \in M_{1}(E \times F)$ denotes the generalized product, defined by

$$
<\eta \otimes^{g e n} \mu_{x}, f>:=\int_{E}\left(\int_{F} f(x, y) \mathrm{d} \mu_{x}(y)\right) \mathrm{d} \eta(x) .
$$

$\eta \otimes^{\text {gen }} \mu_{x}$ is the disintegration of every $\mu \in M_{1}(E \times F)$, where $\eta(B):=|\mu|(B \times F), \forall B \in E$ and $x \mapsto \mu_{x} \in M_{1}(F)$ suitable $\eta$-measurable map. Further $|\mu|=\eta \otimes^{\text {gen }}\left|\mu_{x}\right|$.

$\mu^{\epsilon} \rightarrow_{t s *} \mu^{0}$ two-scale weakly star in $M_{1}(\Omega \times Y)$ iff, for every $\psi \in C_{0}^{0}(\Omega \times Y)$,

$$
\lim _{\epsilon \rightarrow 0} \int_{\Omega} \psi\left(\boldsymbol{x}, \frac{\boldsymbol{x}}{\epsilon}\right) \mathrm{d} \mu^{\epsilon}(\boldsymbol{x})=\int_{\Omega} \int_{Y} \psi(\boldsymbol{x}, \boldsymbol{y}) \mathrm{d} \mu(\boldsymbol{x}, \boldsymbol{y})
$$

where $\left\{\mu^{\epsilon}\right\}$ is a family in $M_{1}(\Omega)$ and $\mu^{0} \in M_{1}(\Omega \times Y)$ (two-scale measure convergence). 


\subsection{Mechanics}

$\Omega$ is a body domain with boundary $\partial \Omega \equiv S$. $S$ has unit normal vector $\boldsymbol{n}$. Moreover, $S=S_{u} \cup S_{t}$ where $S_{u}$ is the part of the boundary that displacements are imposed, and $S_{t}$ is the part of the boundary that tractions are imposed.

$[0, T]$ is the total time interval of the variational problems.

$0=t_{k}^{0}<t_{k}^{0}<t_{k}^{1}<\cdots t_{k}^{k-1}<t_{k}^{k}=T$ denote the $k$ subintervals of the incremental variational problems.

$\boldsymbol{b}(t, \boldsymbol{x}):[0, T] \times \Omega \mapsto \Re^{3}$ denotes the body force vector.

$\boldsymbol{t}(t, \boldsymbol{x}):[0, T] \times S_{t} \mapsto \mathfrak{R}^{3}$ denotes the traction vector.

$\boldsymbol{u}(t, \boldsymbol{x}):[0, T] \times \Omega \mapsto \mathfrak{R}^{3}$ is the displacement vector.

$\boldsymbol{\varepsilon}(t, \boldsymbol{x}):[0, T] \times \Omega \mapsto M_{\mathrm{sym}}^{3 \times 3}$ is the strain tensor with elements $\boldsymbol{\varepsilon}(\boldsymbol{u})=\frac{1}{2}\left[\nabla \boldsymbol{u}+[\nabla(\boldsymbol{u})]^{T}\right]=\operatorname{sym} \nabla \boldsymbol{u}$.

$\boldsymbol{\varepsilon}(\boldsymbol{u})(t, \boldsymbol{x})=\boldsymbol{\varepsilon}^{\mathrm{e}}(t, \boldsymbol{x})+\boldsymbol{\varepsilon}^{\mathrm{p}}(t, \boldsymbol{x})$ in $\Omega, \boldsymbol{u}(t, \boldsymbol{x})=\overline{\boldsymbol{u}}(t, \boldsymbol{x})$ on $S_{u}$ denotes the kinematic admissibility (small strain).

$\boldsymbol{\varepsilon}^{\mathrm{e}}(t, \boldsymbol{x}):[0, T] \times \Omega \mapsto M_{\mathrm{sym}}^{3 \times 3}$ is the elastic strain.

$\boldsymbol{\varepsilon}^{\mathrm{p}}(t, \boldsymbol{x}):[0, T] \times \Omega \mapsto M_{\mathrm{sym}}^{3 \times 3}$ is the plastic strain.

$\boldsymbol{\varepsilon}^{\mathrm{p}}=(\overline{\boldsymbol{u}}-\boldsymbol{u}) \odot \boldsymbol{n} \mathfrak{H}^{2}$ on $S_{u}$ denotes the relaxed boundary condition in perfect plasticity.

$\boldsymbol{e}$ denotes the deviatoric strain.

$\boldsymbol{e}^{\mathrm{e}}$ denotes the deviatoric elastic strain.

$\boldsymbol{\sigma}(t, \boldsymbol{x}):[0, T] \times \Omega \mapsto M_{\mathrm{sym}}^{3 \times 3}$ is the stress tensor.

$\Sigma=\left\{\sigma \in L^{2}\left(\Omega ; M_{\mathrm{sym}}^{3 \times 3}\right): \operatorname{div} \sigma \in L^{3}\left(\Omega ; \Re^{3}\right), s \in L^{\infty}\left(\Omega ; D_{\mathrm{sym}}^{3 \times 3}\right)\right\}$ denotes the admissible stress domain.

$\boldsymbol{\sigma}(t, \boldsymbol{x}) \in K$ where $K$ is the plasticity criterion.

$K=\left\{\sigma \in M_{\mathrm{sym}}^{3 \times 3}: f(\sigma) \leq 0\right\}$ is the strength domain in perfect plasticity.

$f(\boldsymbol{\sigma})=0$ denotes the yield surface.

$s$ denotes the deviatoric stress.

$\sigma_{Y}$ denotes the yield stress in uniaxial tension.

$\check{K}=\left\{\sigma \in M_{\text {sym }}^{3 \times 3}:|s| \leq \sqrt{\frac{2}{3}} \sigma_{Y}\right\}$ is the strength domain in von Mises plasticity.

$N_{K}\left(\xi_{0}\right)$ denotes the normal cone to $K$ at $\xi_{0} \in D_{\text {sym }}^{3 \times 3}$, i.e., if $\xi_{0} \in K$, then $N_{K}\left(\xi_{0}\right)$ is the set of matrices $\zeta \in D_{\text {sym }}^{3 \times 3}$, such that $\zeta:\left(\xi-\xi_{0}\right) \leq 0$ for every $\xi \in K$, otherwise $N_{K}\left(\xi_{0}\right):=\emptyset$.

$[\boldsymbol{\tau}-\boldsymbol{\sigma}(t, \boldsymbol{x})]: \dot{\boldsymbol{\varepsilon}}^{\mathrm{p}} \leq 0, \forall \boldsymbol{\tau} \in K$ denotes associative flow rule.

$\varphi$ or $g$ denotes dissipation potential of viscous materials.

$J_{\mu}$ denotes approximate dissipation potential.

$H\left(\dot{\boldsymbol{\varepsilon}}^{\mathrm{p}}\right), d\left(A^{\mathrm{p}}, A^{\xi}\right)$ denote dissipation potential of perfectly plastic and hardening plastic materials, respectively.

$\dot{\boldsymbol{\varepsilon}}^{\mathrm{p}} \in \partial \varphi(\boldsymbol{\sigma}, \boldsymbol{x})$, where $\varphi(., \boldsymbol{x})$ is lower semicontinuous convex function, and $\partial \varphi(., x)$ is the subdifferential elastoviscoplastic constitutive law. In viscoplastic materials, $\varphi$ is differentiable, and thus, it holds $\dot{\boldsymbol{\varepsilon}}^{\mathrm{p}}=\frac{\partial \varphi}{\partial \boldsymbol{\sigma}}$. 
$B D(\Omega)$ denotes the space of functions with bounded deformation, i.e., the space of all functions in $L^{1}\left(\Re ; \Re^{3}\right)$ such that $\boldsymbol{\varepsilon}(\boldsymbol{u}) \in M_{1}\left(\Omega ; M_{\mathrm{sym}}^{3 \times 3}\right)$.

$\varepsilon(\boldsymbol{u})$.

$\|\boldsymbol{u}\|_{B D(\Omega)}=\int_{\Omega}|\boldsymbol{u}| \mathrm{d} \boldsymbol{x}+\int_{\Omega}|\boldsymbol{\varepsilon}(\boldsymbol{u})|$ is the norm and $|\boldsymbol{\varepsilon}(\boldsymbol{u})|$ is the total variation of the matrix-valued measure

$\prod_{S_{u}}(\Omega)=\varepsilon^{\mathrm{p}} \in M_{1}\left(\Omega \cup S_{u} ; D_{\text {sym }}^{3 \times 3}\right)$ is the space of admissible plastic strains that are bounded Radon measures, for which $\boldsymbol{u} \in B D(\Omega), \overline{\boldsymbol{u}} \in H^{1}\left(\Re^{3} ; \Re^{3}\right), \boldsymbol{\varepsilon}^{\mathrm{e}} \in L^{2}\left(\Omega ; M_{\text {sym }}^{3 \times 3}\right)$ satisfying $\boldsymbol{\varepsilon}(\boldsymbol{u})=\boldsymbol{\varepsilon}^{\mathrm{e}}+\boldsymbol{\varepsilon}^{\mathrm{p}}$ in $\Omega$ and $\boldsymbol{\varepsilon}^{\mathrm{p}}=(\overline{\boldsymbol{u}}-\boldsymbol{u}) \odot \boldsymbol{n} \mathfrak{H}^{2}$ on $S_{u}$.

$<. \mid .>$ denotes duality product for the pairing between a function space and its dual, when the dual is a space of distributions.

$<s(t) \mid \dot{\boldsymbol{\varepsilon}}^{\mathrm{p}}(t)>=<s(t): \dot{\boldsymbol{\varepsilon}}^{\mathrm{p}}(t)>$ denotes the duality product between deviatoric stress and rate of plastic strain.

$\langle\psi\rangle=\frac{1}{\Omega} \int_{\Omega} \psi(\boldsymbol{x}) \mathrm{d} \boldsymbol{x}$ denotes the mean value.

$\mathfrak{X}$ denotes the space of homogenized gradient of the displacement fluctuation with

$$
\mathfrak{X}:=\left\{\boldsymbol{u}^{1} \in M_{1}\left(\Omega \times Y ; \mathfrak{R}^{3}\right): \frac{\partial \boldsymbol{u}^{1}}{\partial \boldsymbol{y}} \in M_{1}\left(\Omega \times Y ; M_{\mathrm{sym}}^{3 \times 3}\right), \boldsymbol{u}^{1}(F \times Y)=0\right.
$$

for every Borel set $F \subseteq \Omega$ \}.

$\boldsymbol{\varepsilon}(\boldsymbol{u})=\boldsymbol{\varepsilon}^{a}(\boldsymbol{u})+\boldsymbol{\varepsilon}^{S}(\boldsymbol{u})=\boldsymbol{\varepsilon}^{a}(\boldsymbol{u})+\boldsymbol{\varepsilon}^{J}(\boldsymbol{u})+\boldsymbol{\varepsilon}^{C}(\boldsymbol{u})$ denotes the decomposition of measure of strain:

- $\boldsymbol{\varepsilon}^{a}(\boldsymbol{u})=\mathfrak{E} \boldsymbol{u} \mathrm{d} \mathfrak{L}^{3}, \mathfrak{E} \boldsymbol{u} \in L^{1}\left(\Omega ; M_{\text {sym }}^{3 \times 3}\right)$ denotes the absolutely continuous part with respect to $\mathfrak{L}^{3}$.

- $\boldsymbol{\varepsilon}^{s}(\boldsymbol{u})$ denotes the singular part.

- $\boldsymbol{\varepsilon}^{J}(\boldsymbol{u})=\llbracket \boldsymbol{u} \rrbracket \odot \boldsymbol{n}_{u} \mathrm{~d} \mathfrak{H}^{2}\lfloor\Gamma$ denotes the jump part, and $\llbracket \boldsymbol{u} \rrbracket$ is the discontinuity across $\Gamma$.

- $\varepsilon^{C}(\boldsymbol{u})$ denotes the Cantor part vanishing on Borel sets which are $\sigma$-finite with respect to the area measure $\mathfrak{H}^{2}$.

\subsection{Elastoplasticity Prandtl-Reuss}

Find $\left(\boldsymbol{u}, \boldsymbol{\varepsilon}^{\mathrm{e}}, \boldsymbol{\varepsilon}^{\mathrm{p}}\right): \Omega \times[0, T] \mapsto \Re^{3} \times M_{\mathrm{sym}}^{3 \times 3} \times M_{\mathrm{sym}}^{3 \times 3}$ such that:

- $\boldsymbol{\varepsilon}=\boldsymbol{\varepsilon}^{\mathrm{e}}+\boldsymbol{\varepsilon}^{\mathrm{p}}$ in $\Omega \times[0, T], \boldsymbol{u}=\boldsymbol{u}^{\mathrm{pr}}$ on $\partial \Omega \times[0, T]$.

- $\sigma=C: \varepsilon^{\mathrm{e}}, \sigma \in K$ in $\Omega \times[0, T]$.

- $\dot{\boldsymbol{\varepsilon}}^{\mathrm{p}} \in N_{K}(\boldsymbol{\sigma})$ in $\Omega \times[0, T]$ or equivalently $\boldsymbol{\sigma}: \dot{\boldsymbol{\varepsilon}}^{\mathrm{p}}=\max _{\boldsymbol{\tau} \in K} \boldsymbol{\tau}: \dot{\boldsymbol{\varepsilon}}^{\mathrm{p}}=: H\left(\dot{\boldsymbol{\varepsilon}}^{\mathrm{p}}\right)$, where $H: M_{\text {sym }}^{3 \times 3} \mapsto \Re$ is the support function of $K$.

- $\left(\boldsymbol{u}(0), \boldsymbol{\varepsilon}^{\mathrm{e}}(0), \boldsymbol{\varepsilon}^{\mathrm{p}}(0)\right)=\left(\boldsymbol{u}_{0}, \boldsymbol{\varepsilon}_{0}^{\mathrm{e}}, \boldsymbol{\varepsilon}_{0}^{\mathrm{p}}\right)$ in $\Omega$.

In dynamic problems $\dot{\boldsymbol{u}}_{0}=\boldsymbol{v}_{0}$.

\section{A review of the existence and uniqueness theorems of plasticity}

It is instructive to recall the milestones in the mathematical literature on plasticity after the pioneering works $[75,86,87,90,107,171]$. The first period commences with the book by Duvaut and Lions [58], who formulated and presented constructive theories, as Galerkin approximations, regularization and penalization methods, for the stress-driven problem, developed in the sequel by Johnson [98-101] and the French school [126, 186,189]. The importance of those works consists in the fact that they combine the theoretical background created by Moreau [145] on the convex analysis with its applications in mechanics. In particular, Moreau [145] provides the proof of existence and uniqueness for the stress problem in elastoplasticity. This period is nicely described by Suquet [188]. The role of dissipation inequality in the stress evolution problem was completely defined, revealing, at the same time, the difficulty of describing the duality between stress and strain in perfect plasticity and the appropriate functional setting for displacement and strain. Independently of this fact, during this period, the appropriate functional setting for the stress, based on a strength control, permitted the development of finite element methods for elastic-perfectly plastic and elastic-hardening models [99, 100]. However, as pointed out by Babadjian [18], "the formulation was too weak for obtaining complete information on the strain tensor". In the sequel, the quasistatic model is studied by Suquet $[187,188]$ and the dynamical model by Anzellotti and Luckhaus [14], as viscoplastic approximations. 
3.1 The cases elastic-perfectly plastic and elastic-viscoplastic: the appropriate functional setting for the stress

This subsection presents the fundamental results of Duvaut and Lions concerning the existence theory of elastoplastic processes, which allow to describe the elastic-perfectly plastic case as the limit case when the viscosity tends to zero.

\subsubsection{Prandtl-Reuss constitutive law}

The constitutive law for smooth (in time) stress history is given by

$$
f(\boldsymbol{\sigma}) \leq 0, \quad \dot{\varepsilon}_{i j}=C_{i j k l}^{-1} \dot{\sigma}_{k l}+\dot{\varepsilon}_{i j}^{\mathrm{p}}, \quad \dot{\varepsilon}_{i j}^{\mathrm{p}}\left[\tau_{i j}-\sigma_{i j}\right] \leq 0, \quad \forall \boldsymbol{\tau} \text { with } f(\boldsymbol{\tau}) \leq 0 .
$$

Here the yield surface $f(\sigma)=0$ defines the plastic zone, $f$ being a convex continuous function in $\sigma_{i j}$, and $C_{i j k l}$ is the elasticity tensor. The operator $\{\dot{\bullet}\}=\frac{\partial\{\bullet\}}{\partial t}$ denotes the partial differentiation with respect to time.

The inequality $(1)_{2}$ satisfied by the incremental plastic strain, is the dissipation inequality expressing the principle of maximum plastic work introduced by Hill. Immediate consequence of this inequality is the normality law $\dot{\varepsilon}_{i j}^{\mathrm{p}} \dot{\sigma}_{i j}=0$.

\subsubsection{Elastic-viscoplastic constitutive law}

This case appears as a fundamental model allowing for obtaining all particular cases: rigid-viscoplastic (elastic coefficients $=0$ ), elastic-perfectly plastic (viscosity $\rightarrow 0$ ), rigid-perfectly plastic (both $\rightarrow 0$ ). The constitutive law reads

$$
\dot{\varepsilon}_{i j}=C_{i j k l}^{-1} \dot{\sigma}_{k l}+\dot{\varepsilon}_{i j}^{\mathrm{p}}, \quad \dot{\varepsilon}_{i j}^{\mathrm{p}} \in \partial J_{\mu}(\boldsymbol{\sigma}), \quad \text { or } \quad J_{\mu}(\boldsymbol{\tau})-J_{\mu}(\boldsymbol{\sigma}) \leq \dot{\varepsilon}_{i j}^{\mathrm{p}}\left[\tau_{i j}-\sigma_{i j}\right] \leq 0, \quad \forall \boldsymbol{\tau} \in \Re^{6},
$$

where $J_{\mu}(\sigma)$ is the potential

$$
J_{\mu}(\boldsymbol{\sigma})=\frac{1}{4 \mu}\left[\tau_{i j}-\left[\boldsymbol{P}_{\tilde{K}} \boldsymbol{\tau}\right]_{i j}\right]\left[\tau_{i j}-\left[\boldsymbol{P}_{\tilde{K}} \boldsymbol{\tau}\right]_{i j}\right]
$$

$\partial J_{\mu}(\boldsymbol{\sigma})$ denotes the subdifferential of $J_{\mu}(\boldsymbol{\sigma})$ and $\boldsymbol{P}_{\tilde{K}} \boldsymbol{\tau}$ is the orthogonal (euclidean) projection of $\boldsymbol{\sigma}$ on the closed convex strength surface $\tilde{K} \subset \mathfrak{R}^{6}$, defined by

$$
\tilde{K}=\left\{\boldsymbol{\sigma} \mid \boldsymbol{\sigma}=\sigma_{i j} \in \Re^{6}, \sigma_{i j}=\sigma_{j i}, f(\boldsymbol{\sigma}) \leq 0\right\} .
$$

Recall that, for functions of several variables $f: U \rightarrow \Re$, where $U$ convex open set $U \subset \Re$, the vector $v$ is called subgradient at $\boldsymbol{x}_{0} \in U$ if, for any $\boldsymbol{x}_{0} \in U$,

$$
f(x)-f\left(x_{0}\right) \geq v \cdot\left[x-x_{0}\right]
$$

The set of $\boldsymbol{v}$ at $\boldsymbol{x}_{0}$ satisfying (5) is called subdifferential and denoted by $\partial f\left(\boldsymbol{x}_{0}\right)$. The subdifferential is a nonempty convex compact set. For the special case of elastic-perfectly plastic materials obeying Tresca or von Mises yield criterium, where $\tilde{K}$ is a subset of the space of symmetric $3 \times 3$ deviatoric tensors including the origin, the indicator function $\mathfrak{I}_{\tilde{K}}$ can be defined. It is worth noticing that, if $\mu \rightarrow 0$, then $J_{\mu} \rightarrow \mathfrak{I}_{\tilde{K}}$ and the constitutive law takes the form (1) of the Prandtl-Reuss law. Finally, it is noted that, according to (2), $\partial J_{\mu}(\sigma)$ is equal to the derivative of $J_{\mu}(\sigma)$ for $\mu>0$. 


\subsubsection{Functional setting of solutions}

Duvaut and Lions [58] studied the (dynamic and static) elastoviscoplastic case of a body $\Omega$ and, under the assumption of steady-state tractions on the boundaries, they proved the existence (and uniqueness) of solutions $\sigma, v=\dot{u}$ such that

$$
\begin{array}{r}
\boldsymbol{\sigma}, \dot{\boldsymbol{\sigma}} \in L^{\infty}\left(0, T ;\left[L^{2}(\Omega)\right]^{6}\right), \\
\boldsymbol{v}, \boldsymbol{v} \in L^{\infty}\left(0, T ;\left[L^{2}(\Omega)\right]^{3}\right), \\
{[\operatorname{div} \boldsymbol{\sigma}]_{i}=\frac{\partial \sigma_{i j}}{\partial x_{j}} \in L^{\infty}\left(0, T ;\left[L^{2}(\Omega)\right]\right),} \\
{[\boldsymbol{\varepsilon}]_{i j}=\frac{1}{2}\left[\frac{\partial v_{i}}{\partial x_{j}}+\frac{\partial v_{j}}{\partial x_{i}}\right] \in L^{\infty}\left(0, T ;\left[L^{2}(\Omega)\right]\right) .}
\end{array}
$$

It is worth noticing that the spatial derivatives of $\sigma$ are not bounded in $L^{\infty}\left(0, T ;\left[L^{2}(\Omega)\right]^{6}\right)$. However, $v$ converges strongly in $L^{\infty}\left(0, T ;\left[L^{2}(\Omega)\right]^{3}\right)$. But the most important point is that there is a complete information on the strain $\varepsilon$, excluding discontinuities during (visco-)plastic deformation.

Passing to the limit for $\mu \rightarrow \infty$ yields the functional setting for the elastic-perfectly plastic case. Duvaut and Lions showed that

$$
\begin{aligned}
\boldsymbol{\sigma}_{\mu}, \dot{\boldsymbol{\sigma}}_{\mu} \rightarrow \boldsymbol{\sigma}, \dot{\boldsymbol{\sigma}} \in L^{\infty}\left(0, T ;\left[L^{2}(\Omega)\right]^{6}\right) & \text { weak star, } \\
\boldsymbol{v}_{\mu}, \dot{\boldsymbol{v}}_{\mu} \rightarrow \boldsymbol{v}, \dot{\boldsymbol{v}} \in L^{\infty}\left(0, T ;\left[L^{2}(\Omega)\right]^{3}\right) & \text { weak star, } \\
\operatorname{div} \boldsymbol{\sigma}_{\mu} \rightarrow \operatorname{div} \boldsymbol{\sigma} \in L^{\infty}\left(0, T ;\left[L^{2}(\Omega)\right]^{3}\right) & \text { weak star, }
\end{aligned}
$$

where $\left(\sigma_{\mu}, \boldsymbol{v}_{\mu}\right)$ and $(\boldsymbol{\sigma}, \boldsymbol{v})$ the solutions of the viscoplastic and elastic-perfectly plastic problems, respectively. It is worth noticing (and clearly cited as a particular remark in [58]) that, in the above functional setting, there is no information on the strain $\varepsilon_{i j}$, in the exception of the fact that $\varepsilon_{i j}$ can be obtained from the stress $\sigma_{i j}$ via $\dot{\varepsilon}_{i j}=C_{i j k h}^{-1} \dot{\sigma}_{k h}+\dot{\varepsilon}_{i j}^{p}$.

\subsection{The elastic-plastic case with hardening}

Johnson [101], inspired by Duvaut and Lions [58] and Halphen and Nguyen [79], formulated the plasticity problem with hardening as a variational inequality and proved the existence (and uniqueness) of a strong solution, as well as the regularity of the displacement rate. The problem of a von Mises material with one isotropic hardening parameter $\xi$, i.e., with $f(\boldsymbol{\sigma}, \xi)=\|\boldsymbol{s}\|-\left(\sigma_{y}+D \xi\right), \quad\|\boldsymbol{s}\|=\left(s_{i j} s_{i j}\right)^{1 / 2}$, where $\boldsymbol{s}$ the deviatoric stress, $\sigma_{y}$ the uniaxial yield stress and $D$ constant, can be stated as:

For displacement described on all $\partial \Omega$, find $((\boldsymbol{\sigma}, \xi), \boldsymbol{v}):[0, T] \mapsto X=\left\{(\boldsymbol{\sigma}, \xi) \in\left[L^{2}(\Omega)\right]^{6} \times L^{2}(\Omega)\right\} \cap$ $\left\{(\sigma, \xi)\right.$ such that $\left.\operatorname{div} \sigma \in\left[L^{2}(\Omega)\right]^{3}\right\}$, for which, a.e. in $[0, T]$,

$$
\begin{aligned}
& \int_{\Omega} \frac{d}{d t}\left(C_{i j k h}^{-1} \sigma_{k h}\right)\left(\tau_{k h}-\sigma_{k h}\right) \mathrm{d} \boldsymbol{x}+D \int_{\Omega} \frac{\partial \xi}{\partial t}(\eta-\xi) \mathrm{d} \boldsymbol{x}+\int_{\Omega} v_{i}\left(\frac{\partial \tau_{i j}}{\partial x_{j}}-\frac{\partial \sigma_{i j}}{\partial x_{j}}\right) \mathrm{d} \boldsymbol{x} \geq 0, \quad \forall(\boldsymbol{\tau}, \eta) \in X, \\
& \int_{\Omega} w_{i} \frac{\partial \sigma_{i j}}{\partial x_{j}} \mathrm{~d} x=\int_{\partial \Omega} b_{i} w_{i} \mathrm{~d} \boldsymbol{x}, \quad \forall \boldsymbol{w} \in\left[L^{2}(\Omega)\right]^{3}, \boldsymbol{\sigma}(0)=\sigma_{0} .
\end{aligned}
$$

Johnson proved that there exists a unique solution of the above problem satisfying

$$
\begin{array}{r}
(\sigma, \xi) \in L^{\infty}\left(\left[L^{2}(\Omega)\right]^{6} \times L^{2}(\Omega)\right), \\
\left(\frac{\partial \sigma}{\partial t}, \frac{\partial \xi}{\partial t}\right) \in L^{2}\left(\left[L^{2}(\Omega)\right]^{6} \times L^{2}(\Omega)\right), \\
\boldsymbol{v} \in\left[L^{2}\left(H_{0}^{1}(\Omega)\right]^{3} .\right.
\end{array}
$$

The theorem is proved via a regularizing version of the system of equations (8) with $\left(\left(\boldsymbol{\sigma}_{\mu \nu}, \xi_{\mu \nu}\right), \boldsymbol{v}_{\mu \nu}\right)$ depending on two parameters $\mu, v$ and the regularizing potential (3). It must be emphasized that stress, hardening parameter, and velocity are uniquely determined thanks to the hardening nature of the material.

The above Sobolev functional setting is ideal for usual finite-element-based or parametric finite-volumebased computational implementation [16,27,76,93,116,162,181,207,210,220]. 


\subsection{Kinematical framework and dissipation}

The second period in the mathematical literature on plasticity is marked by the study of the appropriate functional framework for the velocity and strain rate fields entering the dissipation in order that their product $\dot{\varepsilon}^{\mathrm{p}}: \sigma$ (in other words the duality between strain and stress) makes sense. The key point is the regularity of the strain (and velocity) which is ensured only in viscoplasticity: limit analysis of elastic-perfectly plastic structures proved the existence of discontinuities on the boundaries and/or in $\Omega$. In mechanics-oriented words, in the absence of hardening, the displacement exhibits discontinuities through certain surfaces (slip lines) that cannot be investigated in a Sobolev functional framework. More specifically, plastic strain is only a measure. On the other hand, shear strain localizes into narrow zones forming shear bands which act as precursor to instability and fracture [54,218], while the displacement remains continuous. Matthies et al. [123] and Suquet [186] proposed the framework of functions with bounded deformation $(B D(\Omega)$ in order to describe the velocity and strain rate fields,

$$
B D(\Omega)=\left\{\boldsymbol{v} \mid \boldsymbol{v}=\left(v_{i}\right) ; \boldsymbol{v} \in\left[L^{1}(\Omega)\right]^{3} ; \boldsymbol{\varepsilon} \in M_{1}\left(\Omega ; M_{\mathrm{sym}}^{3 \times 3}\right)\right\},
$$

where $M_{1}\left(\Omega ; M_{\mathrm{sym}}^{3 \times 3}\right)$ is the space of bounded measures on $\Omega$ with values in $M_{\mathrm{sym}}^{3 \times 3}[106,205,206]$. Some regularity properties of this space, namely the integrability of the displacement $u$ and that $u \in\left[L^{p}(\Omega)\right]^{3}$, $p \leq 3 / 2$, are presented by Temam and Strang [206]. A comprehensive collection of their properties, together with the proof of existence of the displacement field of a body obeying Hencky's law, is given by Anzellotti and Giaquinta [13]. Two trace theorems have been established, demonstrating that the space $(B D(\Omega)$ is the most general space for which discontinuities in velocities and strain rates are allowed to appear on the boundaries (boundary conditions) or in the interior of $\Omega$ [123,185]. The above functional setting allowed Suquet [186], following Johnson [98], to present the weak formulation of the evolution problem for an elastic-perfectly plastic material. It is worth noticing that the trace operator in $B D(\Omega)$ is not continuous for some natural weak topology of $B D(\Omega)$, but for a weak-star topology [203]: if $u_{m} \rightarrow u$ for the weak topology of $B D(\Omega)$ and if the total deformation of $u_{m}$ converges to the total deformation of $u$, namely if

$$
\int_{\Omega}\left|\varepsilon_{i j}\left(u_{m}\right)\right| \rightarrow \int_{\Omega}\left|\varepsilon_{i j}(u)\right|,
$$

then the trace of $u_{m}$ converges to the trace of $u$ in the norm of $\left[L^{1}\left(S_{u}\right)\right]^{3}$.

The duality between stresses and strains in the context of the special case of Hencky plasticity, which is valid under proportional loading, is described in [106] (see also [13] for the necessary and sufficient conditions on the external forces for the existence of displacement solutions). However, the mathematical tools used in that paper allow for the extension of proofs of dual pairing between admissible stress and strain to more general plasticity systems [66]. In [106], it is proved the existence of an extremal displacement whenever the safe loading, (i.e., that there exists stress $\sigma$ statically and plastically admissible such that $\lambda \sigma, \lambda>0$ is plastically admissible) holds and that any extremal stress and displacement are related by an optimality condition expressing in weak form the constitutive relation of Hencky plasticity. Hencky derived the first constitutive law in plasticity by assuming the specific complementary energy $A=(1 / 4) \mu \operatorname{tr}\left(\sigma^{2}\right)-[\lambda /(2+\lambda)](\operatorname{tr} \sigma)^{2}$ and the limit of elastic behavior $F=\left(\operatorname{tr}\left(\sigma^{2}\right)-(1 / 3)(\operatorname{tr} \sigma)^{2}-2 k^{2}\right.$ and solved the variational problem by seeking with density of energy $W=A+L u+M v+N w+f F$ for an extremum of the global energy, $L, M, N$ being the balance equations as auxiliary conditions and $f$ a Lagrange multiplier. As a result, he received $\boldsymbol{\epsilon}=(1 / 3) \operatorname{tr} \boldsymbol{I}+[(1+f) / 2 \mu] \tau$, $\operatorname{tr}(\boldsymbol{\epsilon})=(1 / 3 K) \operatorname{tr} \sigma, 3 K=2 \lambda+2 \mu$, where $f$ is a still undetermined Lagrange parameter, and $K$ is the bulk modulus. This formulation is referred to as deformation theory. For many so-called proportional problems this theory represents a very simple method. However, a neutral (i.e., tangential to the limit surface) change of stresses cannot be confronted. For a nice history of plasticity and the role of Hencky, the reader is invited to read the article by Otto Bruhns in [24].

It is known that the Green formula for the integral $\int \sigma: \varepsilon \mathrm{d} \boldsymbol{x}$ is the mathematical expression for the virtual work theorem in the absence of strain discontinuities. In [106] it is defined a pairing $\sigma_{i j} \varepsilon_{i j}$ between admissible stress and strain. They proved that the distribution " $<s_{i j} \varepsilon_{D}(\boldsymbol{u})_{i j}>$ " defined by

$$
\int_{\Omega} s_{i j} \varepsilon_{D}(\boldsymbol{u})_{i j} \phi=-\frac{1}{3} \int_{\Omega}(\operatorname{tr} \boldsymbol{\sigma})(\operatorname{div} \boldsymbol{u}) \phi \mathrm{d} \boldsymbol{x}-\int_{\Omega} \boldsymbol{u} \cdot \operatorname{div} \boldsymbol{\sigma} \phi \mathrm{d} \boldsymbol{x}-\int_{\Omega} \boldsymbol{\sigma}: \nabla \boldsymbol{u} \phi \mathrm{d} \boldsymbol{x}
$$


is a bounded measure, absolutely continuous with respect to the bounded variation $|\varepsilon|$ and satisfying an analogue of Green's formula in the form

$$
\begin{aligned}
\int_{\partial \Omega} \boldsymbol{u} \cdot \boldsymbol{\sigma} \cdot \boldsymbol{n} \psi \mathrm{d} S "=< & \boldsymbol{u} \cdot \boldsymbol{\sigma} \cdot \boldsymbol{n} \phi>=\int_{\Omega} \boldsymbol{s}: \boldsymbol{\varepsilon}_{D}(\boldsymbol{u}) \psi+\frac{1}{3} \int_{\Omega}(\operatorname{div} \boldsymbol{u})(t r \boldsymbol{\sigma}) \psi \mathrm{d} \boldsymbol{x} \\
& +\int_{\Omega}(\boldsymbol{u} \cdot \operatorname{div} \boldsymbol{\sigma}) \psi \mathrm{d} \boldsymbol{x}+\int_{\Omega} \boldsymbol{\sigma}:[\nabla \psi \otimes \boldsymbol{u}] \mathrm{d} \boldsymbol{x}, \quad \forall \psi \in C^{1}(\bar{\Omega}) .
\end{aligned}
$$

Moreover, if $\left\{\boldsymbol{u}_{k}\right\} \subset B D(\Omega), \operatorname{div} \boldsymbol{u}_{k} \in L^{p}(\Omega), 1<p<\infty$, and

$$
\begin{array}{r}
\boldsymbol{u}_{k} \rightarrow \boldsymbol{u} \in L^{\frac{3}{2}}\left(\Omega ; \Re^{3}\right), \operatorname{div} \boldsymbol{u}_{k} \rightarrow \operatorname{div} \boldsymbol{u} \in L^{p}(\Omega), \\
\int_{\Omega}\left|\boldsymbol{\varepsilon}_{D}\left(\boldsymbol{u}_{k}\right)\right| \rightarrow \int_{\Omega}\left|\boldsymbol{\varepsilon}_{D}(\boldsymbol{u})\right|,
\end{array}
$$

then for each $\psi \in C^{0}(\bar{\Omega})$,

$$
\int_{\Omega} \boldsymbol{s}: \boldsymbol{\varepsilon}_{D}\left(u_{k}\right) \psi \rightarrow \int_{\Omega} \boldsymbol{s}: \boldsymbol{\varepsilon}_{D}(u) \psi .
$$

Finally, if $\left\{\boldsymbol{\sigma}_{k}\right\} \subset \Sigma(\Omega)$ converges to $\sigma$ such that

$$
\begin{aligned}
& \sigma_{k} \rightarrow \sigma \in L^{2}\left(\Omega ; M_{\mathrm{sym}}^{3 \times 3}\right), \operatorname{div} \sigma_{k} \rightarrow \operatorname{div} \sigma \in L^{3}\left(\Omega ; \Re^{3}\right), \\
& \left\|\boldsymbol{s}_{k}\right\|_{L^{\infty}}<C \text { indep. of } k,
\end{aligned}
$$

then, for each $\psi \in C^{0}(\bar{\Omega})$,

$$
\int_{\Omega} \boldsymbol{s}_{k}: \boldsymbol{\varepsilon}_{D}(u) \psi \rightarrow \int_{\Omega} \boldsymbol{s}: \boldsymbol{\varepsilon}_{D}(u) \psi
$$

The variational problem of Hencky plasticity is then defined as follows [106]:

Upper-bound problem $(\mathrm{P}): \inf _{u \in \mathfrak{U}} J(\boldsymbol{u})$, where

$$
\begin{aligned}
\mathfrak{U} & =\left\{\boldsymbol{u} \in W^{1,2}\left(\Omega ; \Re^{3}\right): \boldsymbol{u} \cdot \boldsymbol{n}=\overline{\boldsymbol{u}} \cdot \boldsymbol{n} \text { a.e on } S_{u}\right\} \\
J(\boldsymbol{u}) & =\frac{K}{2} \int_{\Omega}|\operatorname{div} \boldsymbol{u}|^{2} \mathrm{~d} \boldsymbol{x}+\int_{\Omega \cup S_{u}} \sup _{\boldsymbol{s} \in K}\left\{\boldsymbol{\varepsilon}_{D}(\tilde{\boldsymbol{u}}): \boldsymbol{s}-\frac{1}{4 \mu}|\boldsymbol{s}|^{2}\right\}-\int_{\Omega} \boldsymbol{u} \cdot \boldsymbol{b} \mathrm{d} \boldsymbol{x}-\int_{S_{t}} \boldsymbol{u} \cdot \boldsymbol{t} \mathrm{d} s .
\end{aligned}
$$

It is worth noticing that the boundary displacement is assumed to be zero outside $S_{u}$ and that the boundary condition is relaxed $(\boldsymbol{u} \cdot \boldsymbol{n}=\overline{\boldsymbol{u}} \cdot \boldsymbol{n})[50,51,171,186,205]$. Moreover, $\tilde{\boldsymbol{u}}$ in $(13)_{2}$ is defined by $\tilde{\boldsymbol{u}}=\boldsymbol{u} \in B D(\Omega)$ on $\Omega, \tilde{\boldsymbol{u}}=U^{0}$ with bounded support on $\Re^{3} \backslash \Omega$.

Lower bound problem (D): $\sup _{\sigma \in \Sigma} G(\sigma)$, where

$$
G(\boldsymbol{\sigma})=-\int_{\Omega}\left(\frac{1}{18 K}|\operatorname{tr} \boldsymbol{\sigma}|^{2}+\frac{1}{4 \mu}|\boldsymbol{s}|^{2}\right) \mathrm{d} \boldsymbol{x}+\int_{S_{u}} \overline{\boldsymbol{u}} \cdot \boldsymbol{\sigma} \cdot \boldsymbol{n} .
$$

Temam and Strang [205] proved that (P) and (D) are dual and in particular that

$$
\inf _{\boldsymbol{u} \in \mathfrak{U}} J(\boldsymbol{u})=\sup _{\boldsymbol{\sigma} \in \Sigma} G(\boldsymbol{\sigma}) .
$$

Additionally, the functional $J(\boldsymbol{u})$ needs to extend to [13,202,204]

$$
\mathfrak{U}^{\dagger}=B D(\Omega) \cap\left\{\boldsymbol{u}: \operatorname{div} \boldsymbol{u} \in L^{2}(\Omega)\right\} \cap\left\{\boldsymbol{u}: \boldsymbol{u} \cdot \boldsymbol{n}=\overline{\boldsymbol{u}} \cdot \boldsymbol{n} \text { on } S_{u}\right\} \subset \subset L^{1}\left(\Omega ; \Re^{3}\right) .
$$

In the above papers, under safe load condition (i.e., that there exists stress $\sigma$ statically and plastically admissible such that $\lambda \sigma, \lambda>0$ is plastically admissible), the existence problem for the displacement problem is reduced to show (i) that $\inf _{\boldsymbol{u} \in \mathfrak{U}^{\dagger}} J(\boldsymbol{u})=\inf _{\boldsymbol{u} \in \mathfrak{U}} J(\boldsymbol{u})$ and (ii) that $J(\boldsymbol{u})$ is lower-semicontinuous under weak convergence in $\mathfrak{U}^{\dagger}$. A new, more general proof [106], is provided for the above existence problem, by introducing the pairing 
between $U(\Omega)=B D(\Omega) \cap\left\{\boldsymbol{u}: \operatorname{div} \boldsymbol{u} \in L^{2}(\Omega)\right\}$ and $\Sigma(\Omega)=L^{2}\left(\Omega ; M_{\mathrm{sym}}^{3 \times 3}\right) \cap\left\{\boldsymbol{\sigma}: \operatorname{div} \boldsymbol{\sigma} \in L^{3}, \quad \boldsymbol{s} \in L^{\infty}\right\}$. More specifically, it is proved the following existence result for displacements:

If the loads $\mathrm{f}, \mathrm{F}$, satisfy the safe load condition, then (P) has a minimizer in $\mathfrak{U}^{\dagger}$,

and the following optimality conditions, satisfied by a pair of extremals $\sigma$ of $(\mathrm{P}), \boldsymbol{\varepsilon}$ of (D):

$$
\begin{gathered}
\int_{\Omega \cup_{u} S} \boldsymbol{\varepsilon}_{D}(\tilde{\boldsymbol{u}}): \boldsymbol{s}-\frac{1}{4 \mu} \int_{\Omega}|\boldsymbol{\sigma}|^{2} \geq \int_{\Omega \cup_{u} S} \boldsymbol{\varepsilon}_{D}(\tilde{\boldsymbol{u}}): \boldsymbol{\tau}-\frac{1}{4 \mu} \int_{\Omega}|\boldsymbol{\tau}|^{2}, \quad \forall \boldsymbol{\tau} \in \Sigma(\Omega), \\
K \operatorname{div} \boldsymbol{u}=\frac{1}{3} \operatorname{tr} \boldsymbol{\sigma} \text { a.e. on } \Omega
\end{gathered}
$$

In case the singular part of deviatoric strain exhibits discontinuities through a surface in the body, as in elastic-perfect plastic materials, an equivalent optimality conditions appear [13,106].

\subsubsection{A functional space for the equations in perfect plasticity}

Suquet [186] introduced a functional space that leads to the existence of a trace on the boundary of $\Omega$ and, equally important, to the possibility of discontinuities in the interior of $\Omega$.

Theorem 3.1 Trace theorem on $\partial \Omega$ : There exists a linear application $\gamma_{-}$from $B D(\Omega)$ on $\left[L^{1}(\Omega)\right]^{3}$ such that, if we write, for $\boldsymbol{u} \in B D(\Omega), \gamma_{-} u=\boldsymbol{u}^{-}$, we have

$$
\begin{aligned}
& \int_{\Omega} u_{i} \frac{\partial \sigma_{i j}}{\partial x_{j}} \mathrm{~d} \boldsymbol{x}+\int_{\Omega} \sigma_{i j} d \varepsilon_{i j}(u) \equiv \int_{\partial \Omega} u_{i}^{-} \sigma_{i j} n_{j} d S \\
& =\int_{\partial \Omega} \frac{1}{2}\left[u_{i}^{-} n_{j}+u_{j}^{-} n_{i}\right] \sigma_{i j} d S, \quad \forall \boldsymbol{\sigma} \in \mathfrak{D}(\bar{\Omega})_{\text {sym }}^{3 \times 3} .
\end{aligned}
$$

Theorem 3.2 Trace theorems on a surface $\Sigma$ in $\Omega$ : Consider a surface $\Sigma$ in the interior of $\Omega$, separating $\Omega$ in two parts $\Omega_{-}$and $\Omega_{+}$. There exist two linear applications $\gamma_{-}$and $\gamma_{+}$from $B D(\Omega)$ on $\left[L^{1}(\Sigma)\right]^{3}$ satisfying

$$
\begin{aligned}
& \int_{\Omega_{-}} u_{i} \frac{\partial \sigma_{i j}}{\partial x_{j}} \mathrm{~d} \boldsymbol{x}+\int_{\Omega_{-}} \sigma_{i j} d \varepsilon_{i j}(u)=\int_{\Sigma} u_{i}^{-} \sigma_{i j} n_{j} d \Sigma, \quad \forall \boldsymbol{\sigma} \in \mathfrak{D}(\bar{\Omega})_{\text {sym }}^{3 \times 3}, \\
& \int_{\Omega_{-}} u_{i} \frac{\partial \sigma_{i j}}{\partial x_{j}} d x+\int_{\bar{\Omega}_{-}} \sigma_{i j} d \varepsilon_{i j}(u)=\int_{\Sigma} u_{i}^{+} \sigma_{i j} n_{j} d \Sigma, \quad \forall \boldsymbol{\sigma} \in \mathfrak{D}(\bar{\Omega})_{\text {sym }}^{3 \times 3} .
\end{aligned}
$$

If $\boldsymbol{u}$ is regular, $\boldsymbol{u}^{-}$and $\boldsymbol{u}^{+}$coincide both with the usual trace of $u$ on $\Sigma$.

Discontinuities through $\Sigma$ : The discontinuity $\boldsymbol{u}^{+}-\boldsymbol{u}^{-}$of $\boldsymbol{u}$ through $\Sigma$ is given by

$$
\int_{\Sigma}\left(u_{i}^{+}-u_{i}^{-}\right) \sigma_{i j} n_{j} \mathrm{~d} \Sigma \equiv \int_{S} \frac{1}{2}\left[\left(u_{i}^{+}-u_{i}^{-}\right) n_{j}+\left(u_{j}^{+}-u_{j}^{-}\right) n_{i}\right] \sigma_{i j} \mathrm{~d} \Sigma=\int_{\Sigma} \sigma_{i j} d \varepsilon_{i j} \mathrm{~d} \Sigma .
$$

So the (possible) mass of $\varepsilon_{i j}(\boldsymbol{u})$ causes a discontinuity $u_{i}^{+}-u_{i}^{-}$through $\Sigma$.

The variational theory of functionals with linear growth uses relaxed boundary conditions for problems involving functions with bounded variation or bounded deformation [47]. In this paper, the total dissipation functional is defined in terms of $\left(\boldsymbol{u}, \boldsymbol{\varepsilon}^{\mathrm{e}}, \boldsymbol{\varepsilon}^{\mathrm{p}}\right), \boldsymbol{u} \in B D(\Omega), \boldsymbol{\varepsilon}^{\mathrm{e}} \in L^{2}\left(\Omega ; M_{\mathrm{sym}}^{3 \times 3}\right), \boldsymbol{\varepsilon}^{\mathrm{p}} \in M^{1}\left(\Omega \cup S_{u} ; D_{\mathrm{sym}}^{3 \times 3}\right)$, subjected to the relaxed boundary conditions involving $\boldsymbol{\varepsilon}^{\mathrm{p}}$ and $\overline{\boldsymbol{u}}(t)$, which is the prescribed boundary value of $\boldsymbol{u}$ on $S_{u} \subset \partial \Omega$ at a time $t$ [see (19)],

$$
\varepsilon_{i j}^{\mathrm{p}}=\frac{1}{2}\left[\left(\bar{u}_{i}(t)-u_{i}\right) n_{j}+\left(\bar{u}_{j}(t)-u_{j}\right) n_{i}\right], \quad \text { on } S_{u} .
$$

Dal Maso et al. [47] provided the mechanical interpretation of this kind of boundary condition: If the prescribed boundary displacement $\bar{u}$ is not attained, a plastic slip develops at the boundary proportional to the difference between the prescribed and the attained boundary displacements.

However, it is worth noticing that in (19) $\sigma$ is regular, while, in perfect plasticity, the stress $\sigma$, the dual of the strain $\varepsilon^{\mathrm{p}}$, is not even continuous. So it is needed to recall two definitions $[47,102]$ which define the duality between stress and plastic strain and allow for using the usual integration by parts formula. This will be clarified in 3.3.3 [Eq. (30)], after presenting the "static" formulation by Suquet [188]. 


\subsubsection{Weak formulation of the elastic-perfectly plastic case}

Find $(\boldsymbol{\sigma}, \boldsymbol{v})$ such that, for every $\boldsymbol{\tau}$ statically and plastically admissible and for every $\boldsymbol{w} \in\left[L^{2}(\Omega)\right]^{3}$,

$$
\begin{gathered}
\int_{\Omega} \frac{d}{d t}\left(C_{i j k h}^{-1} \sigma_{k h}\right)\left(\tau_{k h}-\sigma_{k h}\right) \mathrm{d} \boldsymbol{x}+\int_{\Omega} v_{i}\left(\frac{\partial \tau_{i j}}{\partial x_{j}}-\frac{\partial \sigma_{i j}}{\partial x_{j}}\right) \mathrm{d} \boldsymbol{x} \geq \int_{\partial \Omega_{u}} \bar{v}_{i}\left(\tau_{i j}-\sigma_{i j}\right) n_{j} \mathrm{~d} S, \\
-\int_{\Omega} w_{i} \frac{\partial \sigma_{i j}}{\partial x_{j}} \mathrm{~d} \boldsymbol{x}=\int_{\partial \Omega} b_{i} w_{i} \mathrm{~d} \boldsymbol{x}, \\
\sigma(0)=\sigma_{0} .
\end{gathered}
$$

In order to solve this problem, an additional assumption is necessary [188]: the "limit load," in the sense used in limit analysis of engineering plasticity, is not surpassed. This can be controlled at every step of loading process. Then, the problem of perfect plasticity admits a solution $(\sigma, v)$, unique only in $\sigma$, with

$$
\boldsymbol{\sigma} \in L^{\infty}\left(0, T ;\left[L^{2}(\Omega)\right]_{S}^{9}\right), \quad \dot{\boldsymbol{\sigma}} \in L^{2}\left(0, T ;\left[L^{2}(\Omega)\right]_{s}^{9}\right), \quad \boldsymbol{v} \in L^{2}(0, T ; B D(\Omega)) .
$$

\subsubsection{Stress-strain duality in quasistatic, elastic-perfect plasticity}

The definition of admissible triples $\left(\boldsymbol{u}, \boldsymbol{\varepsilon}^{\mathrm{e}}, \boldsymbol{\varepsilon}^{\mathrm{p}}\right)$ for a given boundary displacement $\overline{\boldsymbol{u}}$ and the definition of a measure $\left[s: \varepsilon^{\mathrm{p}}\right] \in M_{1}\left(\Omega \cup S_{u}\right)$ are recalled now [47,102]:

Admissible triple $\left(\boldsymbol{u}, \boldsymbol{\varepsilon}^{\mathrm{e}}, \boldsymbol{\varepsilon}^{\mathrm{p}}\right)$ : A triple $\left.\left(\boldsymbol{u}, \boldsymbol{\varepsilon}^{\mathrm{e}}, \boldsymbol{\varepsilon}^{\mathrm{p}}\right)\right) \in B D(\Omega) \times L^{2}\left(\Omega ;\left(D^{3 \times 3}\right) \times M_{1}\left(\Omega \cup S_{u} ; D^{3 \times 3}\right)\right.$ is said to be admissible for a given boundary displacement $\overline{\boldsymbol{u}}$ and denoted by $A(\overline{\boldsymbol{u}})$ if the following conditions hold:

(i) additivity: $\boldsymbol{\varepsilon}(\boldsymbol{u})=\boldsymbol{\varepsilon}^{\mathrm{e}}+\boldsymbol{\varepsilon}^{\mathrm{p}}$,

(ii) relaxation (weak form) of boundary conditions: $\varepsilon_{i j}^{\mathrm{p}}=\frac{1}{2}\left[\left(\bar{u}_{i}-u_{i}\right) n_{j}+\left(\bar{u}_{j}-u_{j}\right) n_{i}\right] \mathfrak{h}^{2}$ on $S_{u}$.

Measure $\left[s: \varepsilon^{\mathrm{p}}\right]$ : For $\overline{\boldsymbol{u}} \in W^{1,2}\left(\Omega ; M_{\mathrm{sym}}^{3 \times 3}\right),\left(\boldsymbol{u}, \boldsymbol{\varepsilon}^{\mathrm{e}}, \boldsymbol{\varepsilon}^{\mathrm{p}}\right) \in A(\overline{\boldsymbol{u}})$ and $\boldsymbol{\sigma} \in \Sigma$, the measure $\left[s: \boldsymbol{\varepsilon}^{\mathrm{p}}\right] \in M^{1}\left(\Omega \cup S_{u}\right)$ is defined by the formula

$$
\int_{\Omega \cup S_{u}} \varphi \mathrm{d}\left[\boldsymbol{s}: \boldsymbol{\varepsilon}^{\mathrm{p}}\right]=\int_{\Omega} \varphi \mathrm{d}\left[\boldsymbol{s}: \boldsymbol{\varepsilon}_{D}(\boldsymbol{u})\right]-\int_{\Omega} \varphi \boldsymbol{s}: \boldsymbol{\varepsilon}_{D}^{\mathrm{e}}(\boldsymbol{u}) d x+\int_{\Omega \cup S_{u}} \varphi(\overline{\boldsymbol{u}}-\boldsymbol{u}) \cdot[\boldsymbol{\sigma} \cdot \boldsymbol{n}]^{\perp} \mathrm{d} \mathfrak{h}^{2},
$$

for every $\varphi \in C\left(\Omega \cup S_{u}\right)$. Here $[\boldsymbol{\sigma} \cdot \boldsymbol{n}]^{\perp}$ denotes the tangential part of the traction. Thus the following duality holds [102]:

$$
<\boldsymbol{s}: \boldsymbol{\varepsilon}^{\mathrm{p}}>_{\sum, \Pi}=\left[\boldsymbol{s}: \boldsymbol{\varepsilon}^{\mathrm{p}}\right]\left(\Omega \cup S_{u}\right)=\int_{\Omega \cup S_{u}} \boldsymbol{s}: \mathrm{d} \boldsymbol{\varepsilon}^{\mathrm{p}}
$$

where the measure in the right-hand side is defined by Kohn and Temam [106] and Dal Maso et al. [47]. For the definition of $\Sigma, \Pi$ see Sect. 2. Then the usual integration by parts can be applied to $\left\langle s: \varepsilon^{\mathrm{p}}>\sum, \Pi=\right.$ $\int_{\Omega \cup S_{u}} \boldsymbol{s}: \mathrm{d} \boldsymbol{\varepsilon}^{\mathrm{p}}$ if we write formally $\mathrm{d} \boldsymbol{\varepsilon}=\boldsymbol{\varepsilon}^{\mathrm{e}} \mathrm{d} \boldsymbol{x}+\mathrm{d} \boldsymbol{\varepsilon}^{\mathrm{p}}$ :

$$
\begin{gathered}
\int_{\Omega}\left[\boldsymbol{s}: \boldsymbol{\varepsilon}^{\mathrm{e}}-\boldsymbol{\varepsilon}(\overline{\boldsymbol{u}})\right] \mathrm{d} \boldsymbol{x}=\int_{\Omega} \boldsymbol{s}:\left[\mathrm{d} \boldsymbol{\varepsilon}-\mathrm{d} \boldsymbol{\varepsilon}^{\mathrm{p}}\right]-\int_{\Omega} \boldsymbol{s}: \boldsymbol{\varepsilon}(\overline{\boldsymbol{u}}) \mathrm{d} \boldsymbol{x} \\
=\int_{S_{t}} \boldsymbol{t} \cdot(\boldsymbol{u}-\overline{\boldsymbol{u}}) \mathrm{dh} \mathfrak{h}^{2}+\int_{\Omega} \boldsymbol{b} \cdot(\boldsymbol{u}-\overline{\boldsymbol{u}}) \mathrm{d} \boldsymbol{x}-\int_{\Omega \cup S_{u}} \boldsymbol{s}: \mathrm{d} \boldsymbol{\varepsilon}^{\mathrm{p}},
\end{gathered}
$$

if $\boldsymbol{\sigma} \in \Sigma, \boldsymbol{b} \in L^{3}\left(\Omega ; \Re^{3}\right), \boldsymbol{t} \in L^{\infty}[0, T],\left(\boldsymbol{u}, \boldsymbol{\varepsilon}^{\mathrm{e}}, \boldsymbol{\varepsilon}^{\mathrm{p}}\right) \in A(\overline{\boldsymbol{u}}), \overline{\boldsymbol{u}} \in\left(H^{1}(\Omega)\right)^{3}$, div $\boldsymbol{\sigma}=-\boldsymbol{b}$ a.e. in $\Omega, \boldsymbol{\sigma} \cdot \boldsymbol{n}=\boldsymbol{t}$ on $S_{u}$. This formula implies also an extension of the trace theorem (19) to the case of discontinuous stress $\sigma$. 
3.3.4 Energetic models of rate-independent processes: continuity in time of the quasistatic evolution for elastic-perfectly plastic, Tresca or von Mises materials

A variational formulation expressing the plastic evolution via energy balance and dissipation is introduced by Mielke and coworkers $[122,138]$. This generic theory is useful for phenomena such as dry friction, elastoplasticity, shape memory alloys, quasistatic delamination or fracture [122]. Such systems do not necessarily relax to a complete equilibrium. The energetic models are characterized by internal variables $\boldsymbol{a}$. Internal variables describe the internal state which may consists of plastic deformations, hardening variables, magnetization or phase indicators. In this paper, $\boldsymbol{a}$ stands for the plastic strain $\boldsymbol{\varepsilon}^{p}$ and the hardening parameter $\boldsymbol{\xi}$.

Then the energy storage functional is of the general form

$$
\mathfrak{E}(t, \boldsymbol{u}(t), \boldsymbol{a}(t))=\int_{\Omega} W(\boldsymbol{x}, \operatorname{sym} \nabla \boldsymbol{u}(\boldsymbol{x}), \boldsymbol{a}(\boldsymbol{x})) \mathrm{d} \boldsymbol{x}-<l(t) \mid \boldsymbol{u}>.
$$

In elastoplasticity, the integrand may be of quadratic form in terms of the strain $\boldsymbol{\varepsilon}(\boldsymbol{u})$ and the internal variables $a$,

$$
\mathfrak{E}(t, \boldsymbol{u}(t), \boldsymbol{a}(t))=\frac{1}{2} \int_{\Omega} \boldsymbol{A}:(\operatorname{sym} \nabla \boldsymbol{u} \boldsymbol{a})^{T}:(\operatorname{sym} \nabla \boldsymbol{u} \boldsymbol{a})^{T} \mathrm{~d} \boldsymbol{x}-<l(t) \mid \boldsymbol{u}>.
$$

Additionally, the energy dissipation is given by

$$
D_{\mathfrak{R}}\left(\boldsymbol{a} ; t_{0}, t_{1}\right)=\int_{t_{0}}^{t_{1}} \int_{\Omega} \mathfrak{R}(\boldsymbol{x}, \boldsymbol{a}(t, \boldsymbol{x}), \dot{\boldsymbol{a}}(t, \boldsymbol{x})) \mathrm{d} \boldsymbol{x} \mathrm{d} t
$$

and comes from changes of the internal variables via a dissipation potential $\mathfrak{R}$, which is homogeneous of degree 1 due to rate independency. The theory of generalized standard materials to be presented in the next section, provides the evolution equations (equilibrium equation and generalized forces associated to the internal variables), which result from the dissipation potential by using subdifferential and/or spatial derivatives-see (2) for smooth in time processes. Mielke and coworkers reformulated this problem in a derivative-free energetic forme, based on two conditions, the stability condition (inequality) and the energy balance:

$$
\begin{aligned}
\mathfrak{E}(t, \boldsymbol{u}(t), \boldsymbol{a}(t)) & \leq \mathfrak{E}(t, \tilde{\boldsymbol{u}}, \tilde{\boldsymbol{a}})+\mathfrak{R}(\boldsymbol{a}(t), \dot{\boldsymbol{a}}(t)) \forall \tilde{\boldsymbol{u}}, \tilde{\boldsymbol{a}} \\
\mathfrak{E}(t, \boldsymbol{u}(t), \boldsymbol{a}(t))+D_{\mathfrak{R}}(\boldsymbol{a} ; 0, t) & =\mathfrak{E}(0, \tilde{\boldsymbol{u}}(0), \tilde{\boldsymbol{a}}(0))-\int_{0}^{t}<i(\tau) \mid \boldsymbol{u}(\tau)>\mathrm{d} \tau,
\end{aligned}
$$

where $<l(\tau) \mid \boldsymbol{u}(\tau)>$ is the contribution of external work given by

$$
<l(t) \mid \boldsymbol{u}>:=\int_{\Omega} \boldsymbol{b}(t, \boldsymbol{x}) \cdot \boldsymbol{u}(\boldsymbol{x}) \mathrm{d} \boldsymbol{x}+\int_{S} \boldsymbol{t}(t, \boldsymbol{x}) \cdot \boldsymbol{u}(\boldsymbol{x}) \mathrm{d} \mathfrak{H}^{2}
$$

This theory allows for confronting evolution discontinuities in space or time. In Sect. 8 we present the application of this theory to the homogenization of heterogeneous rate-independent materials. In this subsection, we give the version of this theory introduced by Dal Maso et al. [47], for quasistatic evolution problems in perfect plasticity.

In this theory, the quasistatic evolution problem in a time interval $[0, T]$ under a given inertial force $\boldsymbol{f}(\boldsymbol{x}, t)$ is studied. This problem requires finding functions of displacement, elastic strain, plastic strain, and stress satisfying the usual five conditions of additive decomposition for the strain, the elasticity constitutive equation, the equation of equilibrium, the stress constraint (yield conditions) and the associative flow rule (in the form of the maximum plastic work inequality). The authors use the support function

$$
H(\xi):=\sup _{\zeta \in K} \xi: \zeta
$$


where $K$ is a convex compact neighborhood of 0 in the space of trace free $n \times n$ symmetric matrices, in order to write the associative flow rule in the equivalent forms

$$
\begin{gathered}
\text { normality } \dot{\boldsymbol{\varepsilon}}^{\mathrm{p}} \in N_{K}(\boldsymbol{s}(t, \boldsymbol{x})), \\
\text { dissipation pseudo-potential } \boldsymbol{s}(t, \boldsymbol{x}) \in \partial H\left(\dot{\boldsymbol{\varepsilon}}^{\mathrm{p}}\right), \\
\text { maximum dissipation } H\left(\dot{\boldsymbol{\varepsilon}}^{\mathrm{p}}\right)=\boldsymbol{s}(t, \boldsymbol{x}): \dot{\boldsymbol{\varepsilon}}^{\mathrm{p}}, \\
\text { normal cone to } K \text { at } \xi \text {. In the computational mechanics-orie } \\
\text { on of this type are approximated by the incremental minimization } \boldsymbol{u}_{k}^{i},\left(\boldsymbol{\varepsilon}^{\mathrm{e}}\right)_{k}^{i},\left(\boldsymbol{\varepsilon}^{\mathrm{p}}\right)_{k}^{i} \text { at time } t_{k}^{i}, 0=t_{k}^{0}<t_{k}^{1}<\cdots<t_{k-1}^{i}<t_{k}^{k} \\
\min _{\boldsymbol{u}, \boldsymbol{\varepsilon}^{\mathrm{e}}, \boldsymbol{\varepsilon}^{\mathrm{p}} \in C A\left(w\left(t_{k}^{i}\right)\right)}\left[\mathfrak{Q}\left(\boldsymbol{\varepsilon}^{\mathrm{e}}\right)+\Re\left(\boldsymbol{\varepsilon}^{\mathrm{p}}-\left(\boldsymbol{\varepsilon}^{\mathrm{p}}\right)_{k}^{i-1}\right)-<l\left(t_{k}^{i}\right) \mid \boldsymbol{u}\right)>,
\end{gathered}
$$

where $N_{K}(\boldsymbol{s}(t, \boldsymbol{x}))$ the normal cone to $K$ at $\xi$. In the computational mechanics-oriented literature [16,134, $178,180,207]$, problems of this type are approximated by the incremental minimization problem of defining the approximate solution $\boldsymbol{u}_{k}^{i},\left(\varepsilon^{\mathrm{e}}\right)_{k}^{i},\left(\boldsymbol{\varepsilon}^{\mathrm{p}}\right)_{k}^{i}$ at time $t_{k}^{i}, 0=t_{k}^{0}<t_{k}^{1}<\cdots<t_{k-1}^{i}<t_{k}^{k}=T$, as a minimizer of

where $\mathfrak{Q}\left(\varepsilon^{\mathrm{e}}\right):=\frac{1}{2} \int_{\Omega} \boldsymbol{\varepsilon}^{\mathrm{e}}(\boldsymbol{x}): \boldsymbol{C}: \boldsymbol{\varepsilon}^{\mathrm{e}}(\boldsymbol{x}) \mathrm{d} \boldsymbol{x}$ is the elastic potential, $\mathfrak{R}\left(\dot{\boldsymbol{\varepsilon}}^{\mathrm{p} *}\right):=\int_{\Omega} H\left(\dot{\boldsymbol{\varepsilon}}^{\mathrm{p}}(\boldsymbol{x})\right) \mathrm{d} \boldsymbol{x}$ is the dissipation potential, and $C A(w)$ is the field of kinematically admissible displacements, elastic strain and plastic strain. The stress at $t_{k}^{i}$ is obtained as $\boldsymbol{\sigma}_{k}^{i}=\boldsymbol{C}:\left(\boldsymbol{\varepsilon}^{\mathrm{e}}\right)_{k}^{i}$. As mentioned, the plastic strain belongs to the space of deviatoric tensor-valued bounded Borel measures on $\Omega \times S_{u}$.

Definition of quasistatic evolution : A quasistatic evolution is a function $t \longmapsto\left(\boldsymbol{u}(t), \boldsymbol{\varepsilon}^{\mathrm{e}}(t), \boldsymbol{\varepsilon}^{\mathrm{p}}(t)\right)$ from $t \in[0, T]$ into $B D(\Omega) \times L^{2}\left(\Omega ; M_{\mathrm{sym}}^{3 \times 3}\right) \times M_{1}\left(\Omega \times S_{u} ; D^{3 \times 3}\right)$ which satisfies the following conditions:

Global stability: Ffor every $t \in[0, T]$, we have $\left(\boldsymbol{u}(t), \boldsymbol{\varepsilon}^{\mathrm{e}}(t), \boldsymbol{\varepsilon}^{\mathrm{p}}(t)\right) \in A(\overline{\boldsymbol{u}}(t))$ and

$$
\begin{array}{r}
\mathfrak{Q}\left(\boldsymbol{\varepsilon}^{\mathrm{e}}(t)\right)-<l(t)\left|\boldsymbol{u}(t)>\leq \mathfrak{Q}(\boldsymbol{\eta})+\mathfrak{R}\left(\boldsymbol{q}-\boldsymbol{\varepsilon}^{\mathrm{p}}(t)\right)-<l(t)\right| v>, \\
\text { for every }(\boldsymbol{v}, \boldsymbol{\eta}, \boldsymbol{q} \in A(\overline{\boldsymbol{u}}(t)) ;
\end{array}
$$

Energy balance: The function $t \longmapsto \varepsilon^{\mathrm{p}}(t)$ from $[0, T]$ into $M_{1}\left(\Omega \times S_{u} ; D^{3 \times 3}\right)$ has bounded variation and for every $t \in[0, T]$

$$
\begin{aligned}
& \mathfrak{Q}\left(\boldsymbol{\varepsilon}^{\mathrm{e}}(t)\right)+D_{\mathfrak{R}}\left(\boldsymbol{\varepsilon}^{\mathrm{p}} ; 0, t\right)-<l(t)\left|\boldsymbol{u}(t)>=\mathfrak{Q}\left(\boldsymbol{\varepsilon}^{\mathrm{e}}(0)\right)-<l(0)\right| u(0)> \\
& \quad+\int_{0}^{t}[<\boldsymbol{\sigma}(s): \boldsymbol{\varepsilon}(\overline{\dot{\boldsymbol{u}}}(s))>-<l(s) \mid \overline{\boldsymbol{u}}(s)>] \mathrm{d} s-\int_{0}^{t}\left[<\dot{\boldsymbol{b}}(s)|\boldsymbol{u}(s)>+d<\dot{\boldsymbol{t}}(s)| \boldsymbol{u}(s)>S_{t}\right] \mathrm{d} s,
\end{aligned}
$$

where $A(\overline{\boldsymbol{u}}(t))$ is the set of admissible triples $\left(\boldsymbol{u}, \boldsymbol{\varepsilon}^{\mathrm{e}}, \boldsymbol{\varepsilon}^{\mathrm{p}}\right)$ with $\boldsymbol{\varepsilon}(\boldsymbol{x})=\boldsymbol{\varepsilon}^{\mathrm{e}}(\boldsymbol{x})+\boldsymbol{\varepsilon}^{\mathrm{p}}(\boldsymbol{x})$, for every $\boldsymbol{x} \in \Omega$, such that $\boldsymbol{u}$ satisfies the prescribed boundary condition at $t, \boldsymbol{u}(\boldsymbol{x})=\overline{\boldsymbol{u}}(t, \boldsymbol{x}) \in H^{1}\left(\mathfrak{R}^{3}\right) \times \Re^{3}$ and the relaxed boundary condition $\boldsymbol{\varepsilon}^{\mathrm{p}}(\boldsymbol{x})=(\overline{\boldsymbol{u}}(\boldsymbol{x})-\boldsymbol{u}(\boldsymbol{x})) \odot \boldsymbol{n} \mathfrak{H}^{2}$ on $S_{u}$

and where

$$
D_{\Re}\left(\varepsilon^{\mathrm{p}} ; s, t\right)=\sup \left[\sum_{j=1}^{N} \mathfrak{R}\left(\boldsymbol{\varepsilon}^{\mathrm{p}}\left(t_{j}\right)-\boldsymbol{\varepsilon}^{\mathrm{p}}\left(t_{j-1}\right)\right): s=t_{0} \leq t_{1} \leq \cdots \leq t_{N}=t\right],
$$

and $H\left(\boldsymbol{\varepsilon}^{\mathrm{p}}\right)=\sup _{\sigma \in K}<\boldsymbol{\sigma}: \boldsymbol{\varepsilon}^{\mathrm{p}}>,<\boldsymbol{\sigma}: \boldsymbol{\varepsilon}>=\int_{\Omega} \sigma_{i j} \varepsilon_{i j} \mathrm{~d} \boldsymbol{x}$.

Dal Maso et al. [47] presented the proof of the existence of a quasistatic evolution satisfying the prescribed initial conditions. The same existence result is proved by Johnson [98] and Suquet [188] for a formulation in rate form, leading to a different approximation scheme. The existence theorem [47] is based on the assumptions that the prescribed boundary conditions and body forces are absolutely continuous $(A C)$ in time, so that if the displacement develops sharp discontinuities, this is a result of energy minimization. Moreover, a uniform safe-load history is assumed.

The existence theorem of Dal Maso et al. [47] reads:

If there exists a stable process $\left(\boldsymbol{u}_{0}, \boldsymbol{\varepsilon}_{0}^{\mathrm{e}}, \boldsymbol{\varepsilon}_{0}^{\mathrm{p}}\right) \in A(\overline{\boldsymbol{u}}(0))$, i.e., satisfying the stability condition (42) for the initial data, then there exists a quasistatic evolution $t \mapsto\left(\boldsymbol{u}(t), \boldsymbol{\varepsilon}^{\mathrm{e}}(t), \boldsymbol{\varepsilon}^{\mathrm{p}}(t)\right)$ such that $\boldsymbol{u}(0)=\boldsymbol{u}_{0}, \boldsymbol{\varepsilon}^{\mathrm{e}}(0)=\boldsymbol{\varepsilon}_{0}^{\mathrm{e}}$, 
$\varepsilon^{\mathrm{p}}(0)=\varepsilon_{0}^{\mathrm{p}}$. The proof of this theorem is obtained by a time discretization process, for a sequence $\left(\left(t_{k}^{i}\right)\right)_{0 \leq i \leq k}$ of subdivisions of $[0, T]$. The approximate unknowns $\left(\boldsymbol{u}_{k}^{i},\left(\varepsilon^{\mathrm{e}}\right)_{k}^{i},\left(\varepsilon^{\mathrm{p}}\right)_{k}^{i}\right)$ are defined by induction as a solution to the incremental minimization problem

$$
\min _{\left(\boldsymbol{u}, \boldsymbol{\varepsilon}^{\mathrm{e}}, \boldsymbol{\varepsilon}^{\mathrm{p}}\right) \in A\left(\overline{\boldsymbol{u}}_{k}^{i}\right)}\left\{Q\left(\boldsymbol{\varepsilon}^{\mathrm{e}}\right)+\mathfrak{R}\left(\boldsymbol{\varepsilon}^{\mathrm{p}}-\left(\boldsymbol{\varepsilon}^{\mathrm{p}}\right)_{k}^{i-1}\right)-<l\left(t_{k}^{i}\right) \mid \boldsymbol{u}>\right\},
$$

starting from a set $\left(\boldsymbol{u}_{k}^{0},\left(\boldsymbol{\varepsilon}^{\mathrm{e}}\right)_{k}^{0},\left(\boldsymbol{\varepsilon}^{\mathrm{p}}\right)_{k}^{0}\right)$ belonging to $A(\overline{\boldsymbol{u}}(0))$.

It is again emphasized that every quasistatic evolution is proved to be absolutely continuous in time and that $\boldsymbol{\varepsilon}^{\mathrm{e}}(t)$ and $\boldsymbol{\sigma}(t)$ (but not $\boldsymbol{\varepsilon}^{\mathrm{p}}(t)$ ) are determined uniquely by their initial conditions.

We close this subsection with the results of Sect. 6 of Dal Maso et al. [47], regarding the (strong) classical formulation of the flow rule.

Strong formulation and flow rule: If $\dot{\boldsymbol{\varepsilon}}^{\mathrm{p}}(t) \in L^{2}\left(\Omega ; D^{3 \times 3}\right)$, as in hardening plasticity, then the flow rule reads

$$
\dot{\boldsymbol{\varepsilon}}^{\mathrm{p}}(t, \boldsymbol{x}) \in N_{K}(\boldsymbol{s}(t, \boldsymbol{x})),
$$

where $N_{K}\left(\xi_{0}\right)$ is the normal cone to $K$ at $\xi_{0} \in D^{3 \times 3}$.

In perfect plasticity, where $\dot{\boldsymbol{\varepsilon}}^{\mathrm{p}}(t) \in M_{1}\left(\Omega \cup S_{u} ; D^{3 \times 3}\right)$, Eq. (46) is not correct. The equivalent form reads

$$
\frac{\dot{\boldsymbol{\varepsilon}}^{\mathrm{p}}(t, \boldsymbol{x})}{\left|\dot{\boldsymbol{\varepsilon}}^{\mathrm{p}}(t, \boldsymbol{x})\right|} \in N_{K}(\boldsymbol{s}(t, \boldsymbol{x})), \text { for } \mathfrak{L}^{3}-\text { a.e. } \boldsymbol{x} \in\left\{\left|\dot{\boldsymbol{\varepsilon}}^{\mathrm{p}}(t)\right|>0\right\} \text {. }
$$

We note that the Radon-Nikodym derivative $\frac{\dot{\boldsymbol{\varepsilon}}^{\mathrm{p}}(t, \boldsymbol{x})}{\left|\dot{\boldsymbol{\varepsilon}}^{\mathrm{p}}(t, \boldsymbol{x})\right|}$ of $\dot{\boldsymbol{\varepsilon}}^{\mathrm{p}}(t)$ with respect to its variation $\left|\dot{\boldsymbol{\varepsilon}}^{\mathrm{p}}(t)\right|$ is a function defined $\left|\dot{\boldsymbol{\varepsilon}}^{\mathrm{p}}(t)\right|$-a.e. on $\Omega \cup S_{u}$, so the pointwise formulation of the flow rule cannot be written in the form $\frac{\dot{\boldsymbol{\varepsilon}}^{\mathrm{p}}(t, \boldsymbol{x})}{\left|\dot{\boldsymbol{\varepsilon}}^{\mathrm{p}}(t, \boldsymbol{x})\right|} \in N_{K}(\boldsymbol{s}(t, \boldsymbol{x}))$ since $N_{K}(\boldsymbol{s}(t, \boldsymbol{x}))$ is defined only $\mathfrak{L}^{3}$-a.e. on $\Omega$. Dal Maso et al. [47] introduced a precise representative $\hat{\boldsymbol{s}}(t, \boldsymbol{x})$ of $\boldsymbol{s}(t, \boldsymbol{x})$, defined with respect to the measure $\mu(t):=\mathfrak{L}^{3}+\left|\dot{\boldsymbol{\varepsilon}}^{\mathrm{p}}(t)\right|$, in an unique way for the case that $K$ is strictly convex. More specifically, $\hat{\boldsymbol{s}}$ is obtained as the limit of averages of $\boldsymbol{s}$.

Precise definition of stress: If $K$ is strictly convex, $t \mapsto\left(\boldsymbol{u}, \boldsymbol{\varepsilon}^{\mathrm{e}}, \boldsymbol{\varepsilon}^{\mathrm{p}}\right)(t)$ a quasistatic evolution, $\boldsymbol{\sigma}(t)=$ $C \varepsilon^{\mathrm{e}}(t)$

$$
\boldsymbol{\sigma}^{r}(t, \boldsymbol{x}):=\frac{1}{\mathfrak{L}^{3}(B(\boldsymbol{x}, r) \cap \Omega)} \int_{B(\boldsymbol{x}, r) \cap \Omega} \boldsymbol{\sigma}(t, \boldsymbol{y}) \mathrm{d} \boldsymbol{y}
$$

for every $r>0$ and every $t \in[0, T]$ and for $B(t) \subset \Omega \cup S_{u}$ Borel set,

$$
\begin{aligned}
& \hat{\boldsymbol{s}}(t)=\boldsymbol{s}(t) \mathfrak{L}^{3}-\text { a.e. on } \Omega, \\
& \hat{\boldsymbol{s}}(t)=\partial H\left(\frac{\dot{\boldsymbol{\varepsilon}}^{\mathrm{p}}(t)}{\left|\dot{\boldsymbol{\varepsilon}}^{\mathrm{p}}(t)\right|}\right)\left|\dot{\boldsymbol{\varepsilon}}^{\mathrm{p}}(t)\right|-\text { a.e. on } \Omega \cup S_{u},
\end{aligned}
$$

then

$$
\boldsymbol{s}^{r}(t) \rightarrow \hat{\boldsymbol{s}}(t) \text { strong in } L_{\mu(t)}^{1}\left(\Omega ; D^{3 \times 3}\right) \text { for a.e. } t \in[0, T] .
$$

\section{Classical homogenization of rate-independent materials}

In this section, we present the theoretical framework for a computational scheme, specifically designed for rate-independent materials, based on a multiple plastic potential condition and a maximization of homogenized dissipation, leading to evolution constitutive equations.

Suquet's framework of homogenization of inelastic materials [193] is based on (i) the additivity of mass, internal energy and dissipation and (ii) the localization problem in the unit cell $Y$, for $\boldsymbol{\Sigma}$ (or $\boldsymbol{E}$ ) given, of determining microvariables $\boldsymbol{\sigma}^{0}(\boldsymbol{y}), \boldsymbol{\varepsilon}^{0}(\boldsymbol{y})$ if

microscopic constitutive law is given,

$\operatorname{div} \sigma^{0}=\mathbf{0}$ (microequilibrium),

$<\boldsymbol{\sigma}^{0}>=\boldsymbol{\Sigma} \quad\left(\right.$ or $\left.<\boldsymbol{\varepsilon}\left(\boldsymbol{u}^{0}\right)>=\boldsymbol{E}\right)$,

$\boldsymbol{u}^{0}=\boldsymbol{E} \cdot \boldsymbol{y}+\boldsymbol{u}^{1}, \boldsymbol{u}^{1}$ periodic, and $\boldsymbol{\sigma} \cdot \boldsymbol{n}$ antiperiodic on $\partial Y$, 
where $<f>$ denotes the mean value of $f$ in the unit cell. It is noted that it can be proved that the microstress $\sigma^{0}$ and microstrain $\varepsilon^{0}$ are precisely the two-scale convergence limits of $\boldsymbol{\sigma}^{\epsilon}$ and $\boldsymbol{\varepsilon}^{\epsilon}$ for $\epsilon \rightarrow 0$ [4,156]. Suquet used as internal variable a microplastic strain $\varepsilon^{\mathrm{p} 0}$ entering free energy, plastic potential, microplastic criterion, and microevolution law.

It is worth noticing that, while the macrostrain is given by $\boldsymbol{E}=<\boldsymbol{\varepsilon}^{0}>=<\boldsymbol{\varepsilon}^{\mathrm{e} 0}+\boldsymbol{\varepsilon}^{\mathrm{p} 0}>$, its elastic and plastic parts are given by $\boldsymbol{E}^{\mathrm{e}}=<\boldsymbol{B}^{T}: \varepsilon^{\mathrm{e} 0}>$ and $\boldsymbol{E}^{\mathrm{p}}=<\boldsymbol{B}^{T}: \varepsilon^{\mathrm{p} 0}>$ respectively, where $\boldsymbol{B}$ is the stress concentration tensor defined by $\sigma^{0}=\boldsymbol{B}: \boldsymbol{\Sigma}$ in the absence of plastic deformations. The fact that the plastic macrostrain is not the mean plastic strain defined in the above manner (i.e., satisfying local evolution) was pointed out by Hill [86]. Regarding the homogenized dissipation $D$, it is given in terms of the global and an infinite number of local variables $\boldsymbol{\sigma}^{r}$ (residual stresses (60)) by

$$
D=\boldsymbol{\Sigma}: \dot{\boldsymbol{E}}^{\mathrm{p}}-<\boldsymbol{\sigma}^{r}: \boldsymbol{C}: \dot{\boldsymbol{\sigma}}^{r}>.
$$

Suquet's framework of elastic-perfectly plastic materials consists in: (i) setting the cell problem of a dissipative material with regular potential $\varphi_{\mu}$ for given macroscopic stress history, (ii) next, by using the solution of the microscopic problem in terms of the residual stress $\sigma^{r}$, establishing the homogenized behavior of this intermediate material, and, finally (iii) passing to the limit for $\mu \rightarrow 0$ to obtain the homogenized behavior of the elastic-perfectly plastic material under consideration, in the form

$$
\begin{gathered}
\boldsymbol{S} \in \mathcal{P}, \\
\int_{\Omega} C_{i j k h}^{\mathrm{eff}} \frac{\partial \Sigma_{i j}}{\partial t}\left(T_{k h}-\Sigma_{k h}\right) \mathrm{d} \boldsymbol{x}+\int_{Y} C_{i j k h}(\boldsymbol{y}) \frac{\partial \sigma_{i j}^{r}}{\partial t}\left(\pi_{k h}^{r}-\sigma_{k h}^{r}\right) \mathrm{d} \boldsymbol{y} \geq \int_{\Omega} E_{i j}\left(T_{i j}-\Sigma_{i j}\right) \mathrm{d} \boldsymbol{x}, \\
\forall \mathcal{T}=\left(\boldsymbol{T}, \boldsymbol{\pi}^{r}\right) \in \mathcal{P},
\end{gathered}
$$

where $\boldsymbol{S}=\left(\boldsymbol{\Sigma}, \boldsymbol{\sigma}^{r}\right)$ is the generalized stress, $\boldsymbol{\sigma}^{r}$ is the residual stress defined by

$$
\boldsymbol{\sigma}^{0}(y)=\boldsymbol{B}(\boldsymbol{y}): \boldsymbol{\Sigma}+\boldsymbol{\sigma}^{r}(\boldsymbol{y})
$$

and $\mathcal{P}$ is the convex macroscopic strength surface, introduced by [90] and defined in [189] by

$$
\mathcal{P}=\left\{\mathcal{T},<\boldsymbol{\pi}^{r}>=0, \boldsymbol{B}(\boldsymbol{y}): \boldsymbol{T}+\boldsymbol{\pi}^{r} \in \tilde{K}(\boldsymbol{y}) \forall \boldsymbol{y} \in Y\right\} .
$$

We easily verify the equivalence between microscopic and macroscopic safe loading conditions. However, in order to determine the macroscopic yield surface, we have to know, at a given time t, the whole set $\sigma^{r}$. In other words, it is impossible to eliminate the microscopic level from the macroscopic behavior, like in elasticity. The microscopic level is expressed via an infinite number of residual stresses. It can be shown [193] that the residual stresses can be calculated from a known set of internal microvariables (in this case, $\varepsilon^{\mathrm{p} 0}$ ). Dependence on a infinite number of variables makes the problem practically unimportant, unless it is formulated in terms of an assumed spatial distribution in the cell of the unknown internal variables (piecewise uniform or linear or nonlinear transformation field analysis etc $[29,59,60,65,129,132,133]$. This will be clarified in the next section. However, concerning the limit behavior of the material under a loading history, the theory of limit analysis of structures offers useful tools for computing the limit charge. For such a material, it is easy to show first that the homogenization induces satisfaction of the macroyield condition, i.e., $\sigma^{0} \in K$ implies necessarily $\Sigma \in \mathcal{P}$. Moreover, convexity of the local strength surface implies convexity of the macroscopic strength surface, as has been pointed out by Drucker [57] (convexity is stable by homogenization [193]). The conclusion is that all physical macroscopic stress states must lie within $\mathcal{P}$. The inverse, i.e., that all states $\Sigma \in \mathcal{P}$ are physical macroscopic stress states, is also true, provided that the constituents are elastic-plastic or rigid plastic. It is worth noticing that microdissipation inequality, combined with plasticity criterion in the cell, gives the normality constitutive law, as it will be explained in the next section. In other words, in [193] homogenization of elastic-plastic materials is based on the existence of a microscopic stress $\sigma^{0}$ field associated with $\boldsymbol{\Sigma}$, that necessarily is related to a microscopic plastic strain field $\dot{\boldsymbol{\varepsilon}}^{\mathrm{p}}$ by the normality law.

The link of the above theory with the first computational homogenization techniques is shown by Michel et al. [130], where two different methods are presented, the first based on a finite element scheme and the second on fast Fourier transforms. In the framework of a material made of different rigid-plastic constituents, the effective flow surface is derived by approaching the averaged variational principle via the resolution of a sequence of evolution problems for a composite with elastic-perfectly plastic materials, by giving each 
individual phase an artificial (or real) elastic stiffness $\boldsymbol{C}(\boldsymbol{y})$, since the effective flow surface does not depend on the elastic properties of the constituents. We present the theoretical framework in the sequel. For a strength domain $P$ and a dissipation $d=\sup _{\boldsymbol{\tau} \in P(\boldsymbol{y})} \boldsymbol{\tau}: \dot{\boldsymbol{\varepsilon}}$, the effective dissipation is given by [193]

$$
D(\dot{\boldsymbol{E}})=\sup _{\boldsymbol{\Sigma} \in \mathcal{P}} \boldsymbol{\Sigma}: \dot{\boldsymbol{E}}=\inf _{\boldsymbol{u}^{0} \in \mathcal{K}(\dot{\boldsymbol{E}})}<\mathrm{d}\left(\boldsymbol{\varepsilon}\left(\boldsymbol{u}^{0}\right)\right)>=\inf _{\boldsymbol{u}^{0}} \sup _{\boldsymbol{\tau}}\left(\boldsymbol{\tau}: \dot{\boldsymbol{\varepsilon}}^{0}\right),
$$

where $\mathcal{P}$ the effective strength domain corresponding to statically admissible microstresses lying in $P(\boldsymbol{y})$ and having as mean value $\boldsymbol{\Sigma}$ and $\mathcal{K}$ the set of kinematically admissible displacements obeying (55), possibly enhanced by an incompressibility condition. Adding an artificial elastic stiffness to the rigid-plastic phases as well as an artificial "time" $t$ leads to the evolution cell problem for a strain-driven process (given strain rate $\dot{\boldsymbol{E}}(t)$ ), usually equal to a constant $\dot{E}^{0}$ or for a strain rate to be determined in a stress-driven process,

$$
\begin{aligned}
\dot{\boldsymbol{\sigma}}^{0}(t, \boldsymbol{y}) & =\boldsymbol{C}(\boldsymbol{y}):\left(\boldsymbol{\varepsilon}\left(\dot{\boldsymbol{u}}^{0}(t, \boldsymbol{y})\right)-\dot{\boldsymbol{\varepsilon}}^{\mathrm{p} 0}(t, \boldsymbol{y})\right), \\
\boldsymbol{\sigma}^{0}(t, \boldsymbol{y}) \in P(\boldsymbol{y}) \boldsymbol{\varepsilon}^{\mathrm{p} 0}(t, \boldsymbol{y}) & =\dot{\lambda} \frac{\partial f}{\partial \boldsymbol{\sigma}^{0}}\left(\boldsymbol{y}, \boldsymbol{\sigma}^{0}(t, \boldsymbol{y})\right), \dot{\lambda} \geq 0, \\
\operatorname{div} \boldsymbol{\sigma}^{0}(t, \boldsymbol{y}) & =\mathbf{0}, \boldsymbol{\sigma}^{0} \cdot \boldsymbol{n} \text { antiperiodic, } \\
\dot{\boldsymbol{u}}^{0}(t, \boldsymbol{y}) & =\dot{\boldsymbol{E}}(t) \cdot \boldsymbol{y}+\dot{\boldsymbol{u}}^{1}(t, \boldsymbol{y}), \dot{\boldsymbol{u}}^{1} \text { periodic. }
\end{aligned}
$$

The variational formulation reads: Find $\sigma(t)^{0} \in \mathcal{P}$ such that

$$
<\dot{\boldsymbol{\sigma}}^{0}(t): \boldsymbol{C}^{-1}:\left(\hat{\boldsymbol{\sigma}}-\boldsymbol{\sigma}^{0}(t)\right)>\geq \dot{\boldsymbol{E}}(t):(\hat{\boldsymbol{\Sigma}}-\boldsymbol{\Sigma}(t)), \hat{\boldsymbol{\sigma}} \in \mathcal{P}, \quad \hat{\boldsymbol{\Sigma}}=<\hat{\boldsymbol{\sigma}}>.
$$

In a strain-controlled path, if $\sigma^{0}(t, y)$ tends to $\sigma^{\infty}$ as $t$ goes to $+\infty$, then it is seen from (67) that the associated effective stress $\boldsymbol{\Sigma}^{\infty}$ is on the boundary of the effective strength domain $\mathcal{P}$ and the effective strain rate $\dot{\boldsymbol{E}}$ is an outer normal vector to $\mathcal{P}$ at $\boldsymbol{\Sigma}^{\infty}$. Otherwise, if $\sigma^{0}(t, \boldsymbol{y})$ has no limit, there is no point on the boundary of the effective strength surface associated to the given normal outer vector $\dot{\boldsymbol{E}}$. The integration scheme for a strain path $\dot{\boldsymbol{E}}=t \dot{\boldsymbol{E}}^{0}$ consists in determining the increment of the displacement fluctuation $\Delta \boldsymbol{u}^{1}$ as in the radial mapping algorithm [181] from the nonlinear equilibrium condition by an iterative scheme of Newton's type for a choice of the stiffness matrix, for instance formed with the initial elastic moduli. In an analogous manner, the stress-controlled computational process is presented.

\section{On the role of dissipation inequality in generalized standard models}

For the moment, we assume that the material is a homogeneous generalized standard material (GSM) [74, $79,157,180]$, for which, as cited in the introduction, the local problem is completely described by state and internal variables, while the generalized forces are defined from the free energy function and the dissipation inequality holds giving the evolutionary equations. In classical rate-independent plasticity, the free energy $\psi$ is a function of the internal variables

$$
\psi=\psi(\boldsymbol{\varepsilon}, \boldsymbol{a}),
$$

where $(\boldsymbol{\varepsilon}, \boldsymbol{a})$ are the state variables, $\boldsymbol{\varepsilon}=\boldsymbol{\varepsilon}^{\mathrm{e}}+\boldsymbol{\varepsilon}^{\mathrm{p}}$ is the strain decomposed into an elastic and a plastic part, while $\boldsymbol{a}=\left(\varepsilon^{\mathrm{p}}, \xi\right)$ are the internal variables. Here we assume that the internal variables are the (symmetric in classical plasticity) plastic strain tensor $\varepsilon^{\mathrm{p}}$ and a hardening vector $\xi$. We also assume that the free energy is decomposed into an elastic part (stored energy) $W=\frac{1}{2}\left(\boldsymbol{\varepsilon}-\boldsymbol{\varepsilon}^{\mathrm{p}}\right): \boldsymbol{C}:\left(\boldsymbol{\varepsilon}-\boldsymbol{\varepsilon}^{\mathrm{p}}\right)$ and a plastic part $H^{\mathrm{p}}(\xi)$ which for simplicity is assumed to be independent of the plastic strain,

$$
\psi(\boldsymbol{\varepsilon}, \boldsymbol{a})=\frac{1}{2}\left(\boldsymbol{\varepsilon}-\boldsymbol{\varepsilon}^{\mathrm{p}}\right): \boldsymbol{C}:\left(\boldsymbol{\varepsilon}-\boldsymbol{\varepsilon}^{\mathrm{p}}\right)+H^{\mathrm{p}}(\xi) .
$$

Then, the generalized forces $\boldsymbol{A}=\left(\boldsymbol{A}^{\mathrm{p}}, A^{\xi}\right)$, associated to the internal variables $\boldsymbol{a}=\left(\boldsymbol{\varepsilon}^{\mathrm{p}}, \xi\right)$, are given by

$$
A^{\mathrm{p}}=-\frac{\partial \psi}{\partial \boldsymbol{\varepsilon}^{\mathrm{p}}}=\sigma, \quad A^{\xi}=-\frac{\partial \psi}{\partial \xi}=q,
$$


where $\sigma$ is the stress tensor and $q$ is the internal hardening "force." Thus the plastic criterion can be written

$$
f\left(A^{\mathrm{p}}, A^{\xi}\right) \leq 0 .
$$

Additionally, the dissipation function is given by

$$
d=\sigma: \dot{\boldsymbol{\varepsilon}}-\dot{\psi}=\sigma: \dot{\boldsymbol{\varepsilon}}-\left(\frac{\partial \psi}{\partial \boldsymbol{\varepsilon}}: \dot{\boldsymbol{\varepsilon}}+\frac{\partial \psi}{\partial \boldsymbol{\varepsilon}^{\mathrm{p}}}: \dot{\boldsymbol{\varepsilon}}^{\mathrm{p}}+\frac{\partial \psi}{\partial \xi} \dot{\xi}\right)=\sigma: \dot{\boldsymbol{\varepsilon}}^{\mathrm{p}}-\frac{\partial \psi}{\partial \xi} \dot{\xi}=A^{\mathrm{p}}: \dot{\boldsymbol{\varepsilon}}^{\mathrm{p}}+A^{\xi}: \dot{\xi}=\boldsymbol{A}: \dot{\boldsymbol{a}} .
$$

The dissipation inequality is written

$$
d \geq 0 \text { for every } \boldsymbol{A}^{\mathrm{p}}, A^{\xi} \text { satisfying } f\left(\boldsymbol{A}^{\mathrm{p}}, A^{\xi}\right) \leq 0 .
$$

Then the normality law is obtained simply from (73) by minimizing

$$
-d+\dot{\lambda} f\left(\boldsymbol{A}^{\mathrm{p}}, A^{\xi}\right)
$$

over $\boldsymbol{A}^{\mathrm{p}}, A^{\xi}$, where $\dot{\lambda}$ is a Lagrange multiplier. Therefore, the problem of determination of the evolution equations leads to the problem of minimization of the Lagrangian

$$
\min \mathfrak{L}\left(\boldsymbol{A}^{\mathrm{p}}, A^{\xi}\right)=-\boldsymbol{A}^{\mathrm{p}}: \dot{\boldsymbol{\varepsilon}}^{\mathrm{p}}-A^{\xi}: \dot{\xi}+\dot{\lambda} f\left(\boldsymbol{A}^{\mathrm{p}}, A^{\xi}\right),
$$

from which taking the first variation with respect to $A^{\mathrm{p}}, A^{\xi}$ respectively yields the evolutionary equations

$$
\dot{\boldsymbol{\varepsilon}}^{\mathrm{p}}=\dot{\lambda} \frac{\partial f}{\partial A^{\mathrm{p}}}, \quad \dot{\xi}=\dot{\lambda} \frac{\partial f}{\partial A^{\xi}} .
$$

These equations must be supplemented by the Kuhn-Tucker conditions

$$
f \leq 0, \quad \dot{\lambda} \geq 0, \quad f \dot{\lambda}=0 .
$$

Let us now pass to the verification that the overall behavior of a heterogeneous material is a GSM behavior $[157,193]$. We consider the strain-driven localization problem: For given macrostrain $\boldsymbol{E}$ and internal parameters $\boldsymbol{a}^{0}$ in the unit cell $Y$, find the relation between macrostress $\boldsymbol{\Sigma}$ and macrostrain $\boldsymbol{E}$ if

$$
\begin{gathered}
\boldsymbol{\varepsilon}^{0}=\operatorname{sym} \nabla \boldsymbol{u}^{0} \text { in } Y, \quad<\boldsymbol{\varepsilon}^{0}>=\boldsymbol{E}, \operatorname{div}_{\boldsymbol{y}} \boldsymbol{\sigma}^{0}=0 \text { in } Y, \quad<\boldsymbol{\sigma}^{0}>=\boldsymbol{\Sigma}, \\
\boldsymbol{\sigma}^{0}=\frac{\partial \psi^{0}\left(\boldsymbol{\varepsilon}^{0}, \boldsymbol{a}^{0}\right)}{\partial \boldsymbol{\varepsilon}^{0}}, \psi^{0}\left(\boldsymbol{\varepsilon}^{0}, \boldsymbol{a}^{0}\right)=\frac{1}{2}\left(\boldsymbol{\varepsilon}^{0}-\boldsymbol{\varepsilon}^{\mathrm{p} 0}\right): \boldsymbol{C}^{0}:\left(\boldsymbol{\varepsilon}^{0}-\boldsymbol{\varepsilon}^{\mathrm{p} 0}\right)+H^{\mathrm{p} 0}\left(\xi^{0}\right), \\
\boldsymbol{\sigma}^{0} \text { and } \boldsymbol{\varepsilon}^{0} \text { periodic on } \partial Y .
\end{gathered}
$$

We note that this is an elastic problem giving the solution

$$
\sigma^{0}=\sigma^{0}\left(E, a^{0}\right), \quad \varepsilon^{0}=\varepsilon^{0}\left(E, a^{0}\right), \quad \Sigma=<\sigma^{0}\left(E, a^{0}\right)>.
$$

The main difficulty is that $\boldsymbol{a}^{0}$ must be reduced to a finite-dimensional field, and this issue is the main subject of the computational methods developed by various authors $[59,60,65,129-131]$. In order to determine the effective constitutive behavior of the composite, we assume that the effective free energy satisfies

$$
\Psi=<\psi^{0}\left(\varepsilon^{0}, a^{0}\right)>, \quad \Psi=\Psi\left(\varepsilon^{0}, a^{0}\right) .
$$

A consequence of the above assumption is an application of Hill-Mandel's lemma:

$$
\frac{\partial \Psi}{\partial \boldsymbol{E}}=<\frac{\partial \psi^{0}\left(\boldsymbol{\varepsilon}^{0}, \boldsymbol{a}^{0}\right)}{\partial \boldsymbol{\varepsilon}^{0}}: \frac{\partial \boldsymbol{\varepsilon}^{0}}{\partial \boldsymbol{E}}>=<\boldsymbol{\sigma}^{0}>:<\frac{\partial \boldsymbol{\varepsilon}^{0}}{\partial \boldsymbol{E}}>=\boldsymbol{\Sigma},
$$

where we used that $\frac{\partial \boldsymbol{\varepsilon}^{0}}{\partial \boldsymbol{E}}$ is a admissible strain since, from (55),

$$
\begin{aligned}
& \frac{\partial \boldsymbol{\varepsilon}^{0}}{\partial \boldsymbol{E}}=\frac{\partial}{\partial \boldsymbol{E}}\left(\operatorname{sym} \nabla \boldsymbol{u}^{0}\right)=\operatorname{sym}\left(\nabla \frac{\partial \boldsymbol{u}^{0}}{\partial \boldsymbol{E}}\right) \\
& \frac{\partial \boldsymbol{u}^{0}}{\partial \boldsymbol{E}}=\boldsymbol{I} \cdot \boldsymbol{y}+\frac{\partial \boldsymbol{u}^{1}}{\partial \boldsymbol{E}}, \quad<\frac{\partial \boldsymbol{u}^{1}}{\partial \boldsymbol{E}}>=\frac{\partial}{\partial \boldsymbol{E}}\left(<\boldsymbol{u}^{1}>\right)=0 .
\end{aligned}
$$


We note that from (81) the variation of microstrain is equal to $\delta \boldsymbol{\varepsilon}^{0}=\frac{\partial \boldsymbol{\varepsilon}^{0}}{\partial \boldsymbol{E}}: \delta \boldsymbol{E}^{0}+\frac{\partial \boldsymbol{\varepsilon}^{0}}{\partial \boldsymbol{a}^{0}}: \delta \boldsymbol{a}^{0}$, thus

$$
<\boldsymbol{\sigma}^{0}: \delta \boldsymbol{\varepsilon}^{0}>=<\boldsymbol{\sigma}^{0}>:<\delta \boldsymbol{\varepsilon}^{0}>=<\boldsymbol{\sigma}^{0}>: \delta \boldsymbol{E}+<\boldsymbol{\sigma}^{0}: \frac{\partial \boldsymbol{\varepsilon}^{0}}{\partial \boldsymbol{a}^{0}}: \delta \boldsymbol{a}^{0}>,
$$

therefore

$$
<\boldsymbol{\sigma}^{0}: \frac{\partial \boldsymbol{\varepsilon}^{0}}{\partial \boldsymbol{a}^{0}}: \delta \boldsymbol{a}^{0}>=0
$$

The effective generalized forces $\mathfrak{A}$ are defined in terms of the variation of the effective energy by

$$
\mathfrak{A}: \delta \boldsymbol{a}^{0}=-\frac{\partial \Psi}{\partial \boldsymbol{a}^{0}}: \delta \boldsymbol{a}^{0} .
$$

We note that this definition has a practical meaning only if the field of internal variables $\boldsymbol{a}^{0}$ is approximated by a finite number of variables according to the microstructure, a task which is not trivial.

Using the definition $\psi^{0}=\psi^{0}\left(\boldsymbol{\varepsilon}^{0}, \boldsymbol{a}^{0}\right)$ and (87),

$$
-\frac{\partial \Psi}{\partial \boldsymbol{a}^{0}}: \delta \boldsymbol{a}^{0}=-<\frac{\partial \psi^{0}\left(\boldsymbol{\varepsilon}^{0}, \boldsymbol{a}^{0}\right)}{\partial \boldsymbol{\varepsilon}^{0}}: \frac{\partial \boldsymbol{\varepsilon}^{0}}{\partial \boldsymbol{a}^{0}}: \delta \boldsymbol{a}^{0}>-<\frac{\partial \psi^{0}\left(\boldsymbol{\varepsilon}^{0}, \boldsymbol{a}^{0}\right)}{\partial \boldsymbol{a}^{0}}: \delta \boldsymbol{a}^{0}>=-<\frac{\partial \psi^{0}\left(\boldsymbol{\varepsilon}^{0}, \boldsymbol{a}^{0}\right)}{\partial \boldsymbol{a}^{0}}: \delta \boldsymbol{a}^{0}>.
$$

We conclude that

$$
\mathfrak{A}: \delta \boldsymbol{a}^{0}=<\boldsymbol{A}: \delta \boldsymbol{a}^{0}>.
$$

Based on the above, the overall dissipation $D$ is given by

$$
D=<d>=<\sigma^{0}: \dot{\boldsymbol{\varepsilon}}^{0}-\dot{\psi}^{0}>=<\boldsymbol{A}: \dot{\boldsymbol{a}}^{0}>=\mathfrak{A}: \dot{\boldsymbol{a}}^{0} .
$$

Therefore, the homogenized dissipation inequality can be treated starting from the microstress $\sigma^{0}$ and the rates of the internal microhardening "forces" $\dot{q}^{0}$ and the microplastic strains $\dot{\boldsymbol{\varepsilon}}^{\mathrm{p} 0}$, in correlation with the microyield surface $f^{0}$. This is equivalent to the statement that the microevolutionary equations derived from the microdissipation inequality satisfy the macrodissipation inequality too. This conclusion will be analyzed in the sequel and forms the basis of many computational approaches (see for instance the approach of Tsalis et al. [209]).

\section{Incremental variational homogenization principles for viscous materials}

\subsection{Variational principles}

Numerous authors contributed to the development of variational principles, as well as of incremental variational principles, which allow time integration of constitutive relations along a given strain-driven path. Generally speaking, on a time interval, internal variables as plastic strain and hardening parameter are obtained from the minimization of a functional which comprises the free energy and the dissipation.

Hill [85] presented a variational principle of maximum plastic work for an elastic-perfectly plastic material. In perfect plasticity and hardening plasticity, the fundamental definitions and formulations of the related problems, which affect the uniqueness of their solutions, lead directly to the variational principles as well [56]. Starting form the virtual work, the first absolute minimum principles come from the variation of a quantity by using physical postulates (as Drucker's postulates, for instance that work cannot be extracted from the stressed system and the exterior forces, [56]). Minimum potential energy theorems and minimum complementary energy theorems are established independently of the constitutive law, as well as corresponding theorems in which rates substitute total variations.

The incremental problem in elastoplasticity is a variational problem for elastic strain, plastic strain and hardening parameter. A basic assumption concerns the construction of the dissipation functional in such a way that encodes the information in the yield function and depends on the previous and current values of plastic strain [25]. 
6.2 Two-potential variational problem for generalized standard materials

Ponte-Castañeda [163] proposed a powerful and versatile variational principle, allowing for estimating the effective properties of inelastic materials, mainly focusing on nonlinear viscoelastic response which is very common in polymeric composites. The unknown effective energy function of this material is expressed via the minimization problem of effective energies of linear comparison materials (i.e., materials with linear constitutive laws) having similar microstructure. Then, using known bounds and estimates from linear homogenization yields bounds and estimates for corresponding nonlinear materials.

The idea of defining effective properties (in the context of elasticity) from the effective energy is due to Hill [89] and Hashin [81], based on the (intuitive) notion of homogenized properties that are independent of boundary conditions as the typical size of heterogeneity becomes smaller and smaller.

Following Ponte-Castañeda [164], three categories of methods for predicting effective properties exist: (i) The exact methods for specific microstructures (for instance periodic microstructures) like the cubic lattice [124], the spheres assemblage [84] and the sequentially laminated structures [143]. (ii) The methods for estimating the range of effective properties (bounds) for microstructures partially specified in terms of known heterogeneity parameters ( [84], with bounds which are rederived by $[214,215]$ and other researchers $[104,105$, $119,120,144,149,150,199,216])$. It is noted that Milton [141] has derived effective bounds with a third-order statistical information. (iii) The methods that approximate the essential properties of the microstructure (selfconsistent and other statistical information-based methods [142]). The importance of the existing effective bounds of linear elastic materials is related to the fact that they form a solid data set for comparison materials in the nonlinear variational-based homogenization techniques.

Before the work of Ponte-Castañeda [163], nonlinear heterogeneous dielectrics are studied by Willis [217] and approximate methods, that are generally problem-specific, are proposed, among others, by Miksis [140], Houi and Stroud [94], Zeng et al. [219] and Talbot and Willis in 1985 [194] (with an extension to nonlinear heterogeneous materials of the Hashin-Shtrikman bounds [84]) and 1987 [195] (with bounds of nonlinear dielectrics). After Ponte-Castañeda [163], many theoretical and approximate works are proposed, starting from a variational homogenization principle. In $[20,21]$ the homogenization of elasto-viscoplastic materials with hardening is presented and the variational formulation is compared with the classical radial return algorithm [180]. No yield function is explicitly introduced. Instead, kinematic restrictions for the plastic flow are imposed a priori. The precision is controlled by optimizing an algorithmic parameter in the variational formulation.

In this subsection we assume that the constituents are nonlinear viscoelastic materials and we present the principal ingredients of the two-potential constitutive theory [114,115], which is characterized by the reversibility of the free energy, dependent on the state variables only, the driving forces, resulting from the derivation of the free energy with respect to the state variables, and the irreversibility of the dissipation along an evolution path. The evolution equations are assumed to be derived from a dissipation potential. The constitutive equations can be deduced from two potentials, the free energy and the dissipation potential. An incremental variational principle for a time-discretized evolution equations is presented, at the local level as well as at the level of composite. Time discretization allows for reducing to the minimization of a single potential for a nonlinear thermoelastic body subjected to nonuniform eigenstrains, approximated by piecewise uniform, in each phase, eigenstrains. Moreover, the composite is linearized by a procedure close to the modified secant method [191]: the classic method consists in assuming that, at every phase, the strain is uniform and equal to the mean strain in the phase and then compute the elastic modulus from the constitutive law, while the modified method consists in considering as effective strain of every phase the quadratic mean of strain on this phase, which is proved to coincide with the upper bound introduced by Ponte-Castañeda [163] (see also his work for nonlinear composite dielectrics [164]) and [170]. The material is a nonlinear viscoelastic composite. Since $H^{\mathrm{p}}(\xi)=0$ in (69), $\psi$ and $w$ are identical; therefore, the generalized forces are given by $\boldsymbol{\sigma}=\frac{\partial w}{\partial \boldsymbol{\varepsilon}}(\boldsymbol{\varepsilon}, \boldsymbol{\alpha})$ and $\boldsymbol{A}=-\frac{\partial w}{\partial \boldsymbol{\alpha}}(\boldsymbol{\varepsilon}, \boldsymbol{\alpha})$, where $\boldsymbol{\varepsilon}=\boldsymbol{\varepsilon}^{\mathrm{e}}+\boldsymbol{\alpha}$. The evolution of internal variables are derived from the dissipation potential $\varphi$ by $A=\frac{\partial \varphi}{\partial \dot{\boldsymbol{\alpha}}}(\dot{\boldsymbol{\alpha}})$ or $\dot{\boldsymbol{\alpha}}=\frac{\partial \varphi^{*}}{\partial \boldsymbol{A}}(\boldsymbol{A})$, where $\varphi^{*}$ the Legendre transform of $\varphi$. Elimination of $A$ leads to the two-potential formulation

$$
\boldsymbol{\sigma}=\frac{\partial w}{\partial \boldsymbol{\varepsilon}}(\boldsymbol{\varepsilon}, \boldsymbol{\alpha}), \quad \frac{\partial w}{\partial \boldsymbol{\alpha}}(\boldsymbol{\varepsilon}, \boldsymbol{\alpha})+\frac{\partial \varphi}{\partial \dot{\boldsymbol{\alpha}}}(\dot{\boldsymbol{\alpha}})=0
$$


The discretized version of (92) reads

$$
\begin{array}{r}
\boldsymbol{\sigma}_{n+1}=\frac{\partial w}{\partial \boldsymbol{\varepsilon}}\left(\boldsymbol{\varepsilon}_{n+1}, \boldsymbol{\alpha}_{n+1}\right), \\
\frac{\partial w}{\partial \boldsymbol{\alpha}}\left(\boldsymbol{\varepsilon}_{n+1}, \boldsymbol{\alpha}_{n+1}\right)+\frac{\partial \varphi}{\partial \dot{\boldsymbol{\alpha}}}\left(\frac{\boldsymbol{\alpha}_{n+1}-\boldsymbol{\alpha}_{n}}{\Delta t}\right)=0 .
\end{array}
$$

The second equation is equivalent to the following variational problem, for the unknown $\boldsymbol{\varepsilon}_{n+1}$ and given $\boldsymbol{\sigma}_{n}$, $\boldsymbol{\varepsilon}_{n}, \boldsymbol{\alpha}_{n}$,

$$
\inf _{\alpha} J\left(\boldsymbol{\varepsilon}_{n+1}, \boldsymbol{\alpha}\right), \quad J(\boldsymbol{\varepsilon}, \boldsymbol{\alpha})=w(\boldsymbol{\varepsilon}, \boldsymbol{\alpha})+\Delta t \varphi\left(\frac{\boldsymbol{\alpha}-\boldsymbol{\alpha}_{n}}{\Delta t}\right),
$$

from which, by defining the condensed incremental potential

$$
w_{\Delta}(\boldsymbol{\varepsilon})=\inf _{\boldsymbol{\alpha}} J(\boldsymbol{\varepsilon}, \boldsymbol{\alpha}),
$$

the approximate stress can be obtained as the derivative with respect to the strain [114] of a single potential

$$
\boldsymbol{\sigma}_{n+1}=\frac{\partial w_{\Delta}}{\partial \boldsymbol{\varepsilon}}\left(\boldsymbol{\varepsilon}_{n+1}\right) .
$$

It can be shown that if $w$ and $\varphi$ are convex, then $J$ is convex too [62] (alternatively, see Lahellec and Suquet [114] for twice-differentiable potentials).

Then, the cell problem, consisting in (92), (with respect to $\boldsymbol{\sigma}^{0}, \boldsymbol{\varepsilon}^{0}$ ), $\operatorname{div} \boldsymbol{\sigma}^{0}=\mathbf{0}$ and $<\boldsymbol{\varepsilon}^{0}>=\boldsymbol{E}(t)+$ periodic boundary conditions on $\partial Y$, is discretized as (96), $\operatorname{div} \boldsymbol{\sigma}_{n+1}=0,<\boldsymbol{\varepsilon}_{n+1}>=\boldsymbol{E}_{n+1}$ plus periodic boundary conditions on $\partial Y$. Every cell is comprised of $N$ constituents with characteristic functions $\chi^{(r)}$ and potentials $w^{(r)}$ and $\varphi^{(r)}$, then at a point $\boldsymbol{y}$ the free energy and the dissipation are given by $w(\boldsymbol{y}, \boldsymbol{\varepsilon}, \boldsymbol{\alpha})=\sum_{r=1}^{N} \chi^{r}(\boldsymbol{y}) w^{(r)}(\boldsymbol{\varepsilon}, \boldsymbol{\alpha})$ and $\varphi(\boldsymbol{y}, \dot{\boldsymbol{\alpha}})=\sum_{r=1}^{N} \chi^{r}(\boldsymbol{y}) \varphi^{(r)}(\dot{\boldsymbol{\alpha}})$, respectively. Consequently, the incremental potentials are given by

$$
\begin{aligned}
J(\boldsymbol{\varepsilon}, \boldsymbol{\alpha}, \boldsymbol{y}) & =\sum_{r=1}^{N}\left(w^{(r)}(\boldsymbol{\varepsilon}, \boldsymbol{\alpha})+\Delta t \varphi^{(r)}\left(\frac{\boldsymbol{\alpha}-\boldsymbol{\alpha}_{n}}{\Delta t}\right)\right) \chi^{(r)}(\boldsymbol{y}), \\
w_{\Delta}(\boldsymbol{\varepsilon}, \boldsymbol{y}) & =\inf _{\boldsymbol{\alpha}} J(\boldsymbol{\varepsilon}, \boldsymbol{\alpha}, \boldsymbol{y}) .
\end{aligned}
$$

Lahellec and Suquet [114] have shown that the effective stress is given from the effective energy $\check{w}_{\Delta}$ by

$$
\boldsymbol{\Sigma}_{n+1}=\frac{\partial \check{w}_{\Delta}}{\partial \boldsymbol{E}}\left(\boldsymbol{E}_{n+1}\right)
$$

where $\check{w}_{\Delta}$ is the solution of the variational problem

$$
\check{w}_{\Delta}\left(\boldsymbol{E}_{n+1}\right)=\inf _{<\varepsilon>=\boldsymbol{E}_{n+1}}<w_{\Delta}(\boldsymbol{\varepsilon})>=\inf _{<\boldsymbol{\varepsilon}>=\boldsymbol{E}_{n+1}}<\inf _{\boldsymbol{\alpha}} J(\boldsymbol{\varepsilon}, \boldsymbol{\alpha})>.
$$

The importance of this finding is obvious: The homogenization of the discretized two-potential problem is reduced to the one-potential variational problem (99). However, as is pointed out by Lahellec and Suquet [114], the condensed potential is not explicitly known and is certainly non-quadratic. Moreover, as $w_{\Delta}$ depends on $\boldsymbol{y}$ through $\boldsymbol{\alpha}(\boldsymbol{y}), \boldsymbol{\alpha}_{n}(\boldsymbol{y})$, it cannot be put under the form

$$
w_{\Delta}(\boldsymbol{y}, \boldsymbol{\varepsilon}, \boldsymbol{\alpha})=\sum_{r=1}^{N} \chi^{r}(\boldsymbol{y}) w_{\Delta}^{(r)}(\boldsymbol{\varepsilon}, \boldsymbol{\alpha}) .
$$

Lahellec and Suquet [114] approximate $J$ by replacing in (97) the internal variables $\boldsymbol{\alpha}_{n}(y)$ by an effective (in phase $r$ ) parameter $\boldsymbol{\alpha}_{n}^{(r)}$ to be defined. Dvorak [59] considered all internal variables to be piecewise uniform within each phase (Transformation Field Analysis), but this fact may lead to inaccurate results, as pointed out by Suquet [192] and Chaboche et al. [32]. Thus the approach of Lahellec and Suquet [114] accounts for first and second moments of $\boldsymbol{\alpha}_{n}(\boldsymbol{y}), \boldsymbol{\alpha}(\boldsymbol{y})$ in each phase. Moreover, Lahellec and Suquet approximate $J$ in the variational problem (99) by a quadratic function and present two procedures from the study of one-potential nonlinear 
composites: (i) the variational method of Ponte-Castañeda [165] in its variant introduced by Suquet [191] and Suquet and Ponte-Castañeda [168], which uses an isotropic linear viscoelastic comparison composite, and (ii) a generalization of the second-order method of Ponte-Castañeda [166], introduced by Lahellec and Suquet [112], which uses an anisotropic linear comparison composite.

In the methodology of Lahellec and Suquet [114], two cases of viscous materials can be treated, the nonlinear viscoelastic and the elastic-viscoplastic. They both are characterized by one internal variable tensor $\boldsymbol{\alpha}$ which is the inelastic strain, a quadratic potential energy $w^{(r)}(\boldsymbol{\varepsilon}, \boldsymbol{\alpha})=\frac{1}{2}(\boldsymbol{\varepsilon}-\boldsymbol{\alpha}): \boldsymbol{C}^{(r)}:(\boldsymbol{\varepsilon}-\boldsymbol{\alpha})$ and a dissipation potential of the form $\varphi^{(r)}(\dot{\boldsymbol{\alpha}}) \equiv \varphi^{(r)}\left(\dot{\boldsymbol{\alpha}}_{e q}\right)$, where $\dot{\boldsymbol{\alpha}}_{e q}=\left(\frac{2}{3} \dot{\boldsymbol{\alpha}}: \dot{\boldsymbol{\alpha}}\right)^{1 / 2}$ is the equivalent strain. More specifically, for the nonlinear viscoelastic case,

$$
\varphi^{(r)}(\dot{\boldsymbol{\alpha}})=\frac{\sigma_{Y}^{(r)} \dot{\varepsilon}_{\star}}{m+1}\left(\frac{\dot{\alpha}_{e q}}{\dot{\varepsilon}_{\star}}\right)^{m+1}
$$

with constitutive relation

$$
\dot{\boldsymbol{\varepsilon}}=\left(\boldsymbol{C}^{(r)}\right)^{-1}: \dot{\boldsymbol{\sigma}}+\frac{3}{2} \dot{\varepsilon}_{\star}\left(\frac{\sigma_{e q}}{\sigma_{Y}^{(r)}}\right)^{1 / m} \frac{\boldsymbol{s}}{\sigma_{e q}},
$$

while for the elastic-viscoplastic case,

$$
\begin{gathered}
\varphi^{(r)}(\dot{\boldsymbol{\alpha}})=R^{(r)} \dot{\alpha}_{e q}+\frac{\sigma_{Y}^{(r)} \dot{\varepsilon}_{\star}}{m+1}\left(\frac{\dot{\alpha}_{e_{q}}}{\dot{\varepsilon}_{\star}}\right)^{m+1}, \\
\dot{\boldsymbol{\varepsilon}}=\left(\boldsymbol{L}^{(r)}\right)^{-1}: \dot{\boldsymbol{\sigma}}+\frac{3}{2} \dot{\varepsilon}_{\star}\left(\frac{\left(\sigma_{e q}-R^{(r)}\right)^{+}}{\sigma_{Y}^{(r)}}\right)^{1 / m} \frac{\boldsymbol{s}}{\sigma_{e q}},
\end{gathered}
$$

where $\sigma_{Y}$ is the yield stress, $\dot{\varepsilon}_{\star}$ is a reference strain rate and $R^{(r)}$ is the threshold below which the material behaves elastically. The limiting case $m=0$ corresponds to the rate-independent elastoplasticity.

Lahellec and Suquet [114], inspired by Ponte-Castañeda [165], proposed a variational method in order to give a way of estimating the effective incremental potential. This is achieved by adding and subtracting to the potential $J$ the quadratic energy $J_{0}$ of the linear composite. Then the difference $J-J_{0}$ is estimated by using the finding of Ponte-Castañeda and Willis [169] and Ponte-Castañeda [167] that the sharper estimate can be obtained by requiring only stationarity of $J-J_{0}$, since the supremum of $J-J_{0}$ is too stiff [113]. For a composite made of linear viscoelastic constituents with dissipation potential $\varphi^{(r)}(\dot{\boldsymbol{\alpha}})=\eta^{(r)} \dot{\boldsymbol{\alpha}}: \dot{\boldsymbol{\alpha}}$, the estimate for $\breve{w}_{\Delta}$ is [114]

$$
\check{w}_{\Delta}(\boldsymbol{E})=\check{w}_{0}(\boldsymbol{E})+\sum_{r=1}^{N} c^{(r)}<\frac{\eta^{(r)} \theta^{(r)}}{\Delta t\left(\theta^{(r)}-1\right)}\left(\boldsymbol{\alpha}_{n}-\boldsymbol{\alpha}_{n}^{(r)}\right):\left(\boldsymbol{\alpha}_{n}-\boldsymbol{\alpha}_{n}^{(r)}\right)>_{r},
$$

where $c^{(r)}$ is the volume fraction, $<\#>_{r}$ denotes averaging over the phase $r, \theta^{(r)}=\frac{\eta_{0}^{(r)}}{\eta^{(r)}}$ and $\tilde{w}_{0}(\boldsymbol{E})$ is the effective energy of the auxiliary thermoelastic composite [113]

$$
\check{w}_{0}(\boldsymbol{E})=\inf _{<\boldsymbol{\varepsilon}>=\boldsymbol{E}} \sum_{r=1}^{N} c^{(r)}<w_{0}^{(r)}(\boldsymbol{\varepsilon})>_{r}, \quad w_{0}^{(r)}(\boldsymbol{\varepsilon})=\inf _{\boldsymbol{\alpha}} J_{0}^{(r)}(\boldsymbol{\varepsilon}, \boldsymbol{\alpha}) .
$$

Moreover, the overall stress reads

$$
\boldsymbol{\Sigma}=\frac{\partial \check{w}_{\Delta}}{\partial \boldsymbol{E}}(\boldsymbol{E})=\frac{\partial \check{w}_{0}}{\partial \boldsymbol{E}}(\boldsymbol{E})
$$

due to the stationarity conditions on $\theta^{(r)}, \boldsymbol{\alpha}_{n}^{(r)}, \boldsymbol{\alpha}$. 
For the general nonlinear case with a non-quadratic potential $\varphi$, it is shown that this problem is reduced to that of a linear viscoelastic case provided that the present $\eta^{(r)}$ 's are the "effective secant viscosities" [114]

$$
\eta^{(r)}=\eta_{s c t}^{(r)} \frac{<\left(\alpha-\alpha_{n}\right)_{e q}^{2}>_{r}^{1 / 2}}{\Delta t}
$$

each of which is a nonlinear equation since the internal variable tensor $\boldsymbol{\alpha}\left(\eta^{(r)}\right)$ depends on $\eta^{(r)}$ and is solved iteratively.

Equation (106) indicates that the effective stress coincides with the effective stress of the linear thermoelastic composite. This is given by the following expression

$$
\boldsymbol{\Sigma}=\sum_{r=1}^{n} c^{(r)}\left[\mathcal{C}_{0}^{(r)}:<\boldsymbol{\varepsilon}>_{r}+\boldsymbol{\rho}_{0}^{(r)}\right]=<\boldsymbol{\sigma}>,
$$

where the tensors $\mathcal{C}_{0}^{(r)}$ and $\boldsymbol{\rho}_{0}^{(r)}$ are uniform in every phase $r$ and depend on the fourth-order tensor $\boldsymbol{K}$ associated with the projection over deviatoric tensors (see Lahellec and Suquet [114] for the analytical expression), on the fourth-order tensor $\boldsymbol{C}$ of elasticity in phase $r$ and on the parameters $\eta^{(r)}, \theta^{(r)}, \boldsymbol{\alpha}_{n}^{(r)}$, where $\boldsymbol{\varepsilon}, \boldsymbol{\sigma}$ correspond to the auxiliary thermoelastic problem. This is equivalent to say that, in view of determining the overall stress, the unknown stress and strain are approximated by the same fields of the linear thermoelastic problem [114]. Moreover, it follows from the stationarity conditions for $\theta^{(r)}, \boldsymbol{\alpha}^{(r)}$ that the first and second moments of $\boldsymbol{\alpha}$ take the same values by the two different variational approaches, thus the approximate field of internal variables $\boldsymbol{\alpha}$ is uniquely defined, without ambiguity too for the full field $\boldsymbol{\alpha}(\boldsymbol{y})$, which is not needed for the implementation of the computational scheme.

\section{A discretized variational framework}

Simo and Hughes [180] proposed a discrete variational framework of the $\mathrm{Hu}-$ Washizu type. The variational formulation in the form of minimization problem uses a Hu-Washizu functional enriched with the total dissipation function. It is assumed that the flow law and loading-unloading conditions hold pointwise. It is tested independently at each quadrature point of the element whether the elastic trial state violates the yield condition. If yes, one applies a local return mapping algorithm. The validity of the computational procedure depends on the strain-dependent yield condition.

\subsection{The Hu-Washizu-Simo-Hughes variational problem}

Here we will adopt this variational framework in order to pass to the limit in the discrete Euler-Lagrange equations for $\epsilon \rightarrow 0$. The limiting equations will give information on the weak properties of all microvariables, including microinternal variables. We note that Terada and Kikuchi [207] considered the dissipation inequality as a separate maximum dissipation condition. This inequality is nonlinear thus not allowing for passing to the limit with the help of a Lagrange multiplier in order to determine the local microevolutionary equations.

The body under consideration is submitted to body forces $\boldsymbol{b}$, while the boundary conditions are described by zero displacements on a part $S_{u}^{\epsilon}$ of the surface and tractions $t$ on the remaining part $S_{t}^{\epsilon}$. In the sequel, for simplicity we will consider a material with linear hardening $\left(H^{\mathrm{p} \epsilon}\left(\xi^{\epsilon}\right)=\frac{1}{2} \xi^{\epsilon} D^{\epsilon} \xi^{\epsilon}\right)$ with free function

$$
\psi\left(\boldsymbol{\varepsilon}^{\epsilon}-\boldsymbol{\varepsilon}^{\mathrm{p} \epsilon}, q^{\epsilon}\right)=w\left(\boldsymbol{\varepsilon}^{\epsilon}-\boldsymbol{\varepsilon}^{\mathrm{p} \epsilon}\right)+\frac{1}{2} q^{\epsilon}\left(D^{\epsilon}\right)^{-1} q^{\epsilon},
$$

where $D^{\epsilon}$ is the scalar plastic modulus. We recall that, in the case of linear hardening, heterogeneous functions satisfy the following equations

$$
\begin{aligned}
& f^{\epsilon}\left(\boldsymbol{\sigma}^{\epsilon}, q^{\epsilon}\right)=\left\|\boldsymbol{s}^{\epsilon}\right\|+q^{\epsilon}-\sqrt{\frac{2}{3}} \sigma_{Y}^{\epsilon} \leq 0, \quad q^{\epsilon}=-D^{\epsilon} \xi^{\epsilon}, \\
& \dot{\xi} \epsilon=\left\|\dot{\boldsymbol{\varepsilon}}^{\mathrm{p} \epsilon}\right\| \sqrt{\frac{2}{3}}=\dot{\lambda}^{\epsilon}, \quad \dot{\varepsilon}_{i j}^{\mathrm{p} \epsilon}=\dot{\lambda}^{\epsilon} \frac{\partial f^{\epsilon}}{\partial \sigma_{i j}^{\epsilon}}, \\
& \dot{\varepsilon}_{i j}^{\epsilon}=\dot{\varepsilon}_{i j}^{\mathrm{e} \epsilon}+\dot{\varepsilon}_{i j}^{\mathrm{p} \epsilon}, \quad \dot{\varepsilon}_{i j}^{\mathrm{e} \epsilon}=C_{i j k l}^{\epsilon-1} \dot{\sigma}_{k l}^{\epsilon} .
\end{aligned}
$$


We also note that the choice of linear hardening and $(110)_{2}$ are equivalent to the second of (70).

The total Hu-Washizu energy is given by

$$
\begin{aligned}
\mathfrak{P}^{\epsilon}\left(\boldsymbol{u}^{\epsilon}, \boldsymbol{\varepsilon}^{\epsilon}-\boldsymbol{\varepsilon}^{\mathrm{p} \epsilon}, q^{\epsilon}, \boldsymbol{\sigma}^{\epsilon}\right)= & \int_{\Omega^{\epsilon}}\left[\psi\left(\boldsymbol{\varepsilon}^{\epsilon}-\boldsymbol{\varepsilon}^{\mathrm{p} \epsilon}, q^{\epsilon}\right)+\boldsymbol{\sigma}^{\epsilon}:\left[\operatorname{sym} \nabla \boldsymbol{u}^{\epsilon}-\boldsymbol{\varepsilon}^{\epsilon}\right]\right] \mathrm{d} \boldsymbol{x} \\
& -\int_{\Omega^{\epsilon}} \boldsymbol{b}^{\epsilon} \cdot \boldsymbol{u}^{\epsilon} \mathrm{d} \boldsymbol{x}-\int_{S_{t}^{\epsilon}} \boldsymbol{t} \cdot \boldsymbol{u}^{\epsilon} \mathrm{d} S .
\end{aligned}
$$

In (111) $\boldsymbol{u}^{\epsilon}, \boldsymbol{\varepsilon}^{\epsilon}, \boldsymbol{\sigma}^{\epsilon}, q^{\epsilon}$ are considered as independent variables. In this context, $\boldsymbol{\sigma}^{\epsilon}$ acts as Lagrange multiplier enforcing (weakly) $\operatorname{sym} \nabla \boldsymbol{u}-\boldsymbol{\varepsilon}=0$. Additionally, the plastic dissipation functional reads

$$
\mathfrak{L}^{\epsilon}\left(\boldsymbol{\varepsilon}^{\epsilon}-\boldsymbol{\varepsilon}^{\mathrm{p} \epsilon}, q^{\epsilon}, \lambda^{\epsilon}\right)=\int_{0}^{t} \int_{\Omega^{\epsilon}} \dot{\boldsymbol{\varepsilon}}^{\mathrm{p} \epsilon}: \nabla w^{\epsilon}\left(\boldsymbol{\varepsilon}^{\epsilon}-\boldsymbol{\varepsilon}^{\mathrm{p} \epsilon}\right)-\dot{q}^{\epsilon}\left(D^{\epsilon}\right)^{-1} q^{\epsilon}-\dot{\lambda}^{\epsilon} f^{\epsilon}\left[\nabla w^{\epsilon}\left(\boldsymbol{\varepsilon}^{\epsilon}-\boldsymbol{\varepsilon}^{\mathrm{p} \epsilon}\right), q^{\epsilon}\right] \mathrm{d} \boldsymbol{x} d \tau
$$

where $\dot{\lambda}^{\epsilon}$ is the plastic multiplier and $f^{\epsilon}$ is the yield surface.

We discretize the time interval $[0, T]=\bigcup_{n=0}^{N}\left[t_{n}, t_{n+1}\right]$ and denote by $\mathbb{V}$ the space of kinematically admissible displacement variations $\boldsymbol{\eta}^{\epsilon}$,

$$
\mathbb{V}=\eta^{\epsilon}: \Omega^{\epsilon} \longmapsto \Re^{3} \mid \eta \in H^{1}\left(\Omega^{\epsilon}\right)^{3} ; \eta^{\epsilon}=0 \text { on } S_{u}^{\epsilon}, \boldsymbol{u}^{\epsilon}=0 \text { on } S_{u}^{\epsilon}, \boldsymbol{\sigma} \cdot \boldsymbol{n}=\boldsymbol{t} \text { on } S_{t}^{\epsilon},
$$

corresponding to a problem with displacements $\boldsymbol{u}^{\epsilon}$ and stress $\sigma^{\epsilon}$ satisfying the boundary conditions,

$$
\boldsymbol{u}^{\epsilon}=0 \text { on } S_{u}^{\epsilon}, \boldsymbol{\sigma} \cdot \boldsymbol{n}=\boldsymbol{t} \text { on } S_{t}^{\epsilon} .
$$

We will express the total energy at time $t_{n+1}$ in terms of the unknown state variables $\left(\boldsymbol{\varepsilon}_{n+1}^{\epsilon}, \boldsymbol{\varepsilon}_{n+1}^{\mathrm{p} \epsilon}, q_{n+1}^{\epsilon}\right)$, as the sum of the potential energy at $t_{n+1}$ and the incremental dissipation in the interval $\left[t_{n}, t_{n+1}\right]$ computed by a backward Euler difference scheme. Then we will obtain a discrete Lagrangian in terms of the unknown variables. For details, see the work of Simo and Hughes [180]. In the sequel, by $\varphi_{n+1}$ we denote the approximation to the exact $\varphi\left(t_{n+1}\right)$ at $t_{n+1}$. Following the approach of Simo and Hughes [180], we use the following notations

$$
\begin{array}{r}
\chi_{n+1}^{\epsilon}=\left(\boldsymbol{u}_{n+1}^{\epsilon}, \boldsymbol{\varepsilon}_{n+1}^{\epsilon}, \boldsymbol{\sigma}_{n+1}^{\epsilon}, \boldsymbol{\varepsilon}_{n+1}^{\mathrm{p} \epsilon}, q_{n+1}^{\epsilon}, \Delta \lambda\right), \quad \Delta \lambda=\dot{\lambda}_{n+1}^{\epsilon} \Delta t, \\
w_{n+1}^{\epsilon}=w^{\epsilon}\left(\boldsymbol{\varepsilon}_{n+1}^{\epsilon}-\boldsymbol{\varepsilon}_{n+1}^{\mathrm{p} \epsilon}\right), \quad f_{n+1}^{\epsilon}=f^{\epsilon}\left(\nabla w^{\epsilon}\left(\boldsymbol{\varepsilon}_{n+1}^{\epsilon}-\boldsymbol{\varepsilon}_{n+1}^{\mathrm{p} \epsilon}\right), q_{n+1}^{\epsilon}\right) .
\end{array}
$$

Then we obtain the minimization problem of the discrete energy functional

$$
\mathfrak{F}^{\epsilon}\left(\chi_{n+1}\right)=\mathfrak{P}_{n+1}^{\epsilon}\left(\chi_{n+1}\right)+\mathfrak{L}^{\epsilon}\left(\chi_{n+1}\right)-\mathfrak{L}^{\epsilon}\left(\chi_{n}\right)
$$

or

$$
\begin{aligned}
& \mathfrak{F}^{\epsilon}\left(\chi_{n+1}\right)=\int_{\Omega^{\epsilon}}\left[w_{n+1}^{\epsilon}+\frac{1}{2} q_{n+1}^{\epsilon}\left(D^{\epsilon}\right)^{-1} q_{n+1}^{\epsilon}+\boldsymbol{\sigma}_{n+1}^{\epsilon}:\left(\operatorname{sym} \nabla \boldsymbol{u}_{n+1}^{\epsilon}-\boldsymbol{\varepsilon}_{n+1}^{\epsilon}\right)-\Delta \lambda f_{n+1}^{\epsilon}\right. \\
& \left.+\nabla w_{n+1}^{\epsilon}:\left(\boldsymbol{\varepsilon}_{n+1}^{\mathrm{p} \epsilon}-\boldsymbol{\varepsilon}_{n}^{\mathrm{p} \epsilon}\right)-q_{n+1}^{\epsilon}\left(D^{\epsilon}\right)^{-1}\left(q_{n+1}^{\epsilon}-q_{n}^{\epsilon}\right)-\rho^{\epsilon} \boldsymbol{b}^{\epsilon} \cdot \boldsymbol{u}_{n+1}^{\epsilon}\right] \mathrm{d} \boldsymbol{x}-\int_{S_{t}^{\epsilon}} \boldsymbol{t} \cdot \boldsymbol{u}_{n+1}^{\epsilon} \mathrm{d} S .
\end{aligned}
$$

Using the functional spaces

$$
\begin{aligned}
\mathbb{V}_{\sigma} & =\left(\sigma^{\epsilon}: \Omega^{\epsilon} \longmapsto M_{s y m}^{3 \times 3} \mid \sigma_{i j} \in L^{2}\left(\Omega^{\epsilon}\right)\right), \\
\mathbb{V}_{\epsilon} & =\left(\varepsilon^{\epsilon}: \Omega^{\epsilon} \longmapsto M_{s y m}^{3 \times 3} \mid \varepsilon_{i j} \in L^{2}\left(\Omega^{\epsilon}\right)\right), \\
\mathbb{V}_{q} & =\left(q^{\epsilon}: \Omega^{\epsilon} \longmapsto \Re \mid q \in L^{2}\left(\Omega^{\epsilon}\right)\right), \\
\mathbb{V}_{\lambda} & =\left(\lambda^{\epsilon}: \Omega^{\epsilon} \longmapsto \Re \mid \lambda^{\epsilon} \in L^{2}\left(\Omega^{\epsilon}\right)\right),
\end{aligned}
$$


we can obtain the variational form of the equations of the heterogeneous problem from the Euler-Lagrange equations associated with the discrete functional (117). More specifically, by considering $\left(\chi_{n+1}\right)$ as independent variables, we take the variations of (117) with respect to $\boldsymbol{u}_{n+1}^{\epsilon}, \boldsymbol{\varepsilon}_{n+1}^{\epsilon}, \boldsymbol{\sigma}_{n+1}^{\epsilon}, \boldsymbol{\varepsilon}_{n+1}^{\mathrm{p} \epsilon}, q_{n+1}^{\epsilon}$ and $\Delta \lambda$ successively thus obtaining

$$
\begin{aligned}
& \delta \mathfrak{F}^{\epsilon}\left(\chi_{n+1}, \boldsymbol{\eta}^{\epsilon}\right)=\int_{\Omega^{\epsilon}}\left[\boldsymbol{\sigma}_{n+1}^{\epsilon}: \operatorname{sym} \nabla \boldsymbol{\eta}^{\epsilon}-\rho^{\epsilon} \boldsymbol{b} \cdot \boldsymbol{\eta}^{\epsilon}\right] \mathrm{d} \boldsymbol{x}-\int_{S_{t}^{\epsilon}} \boldsymbol{t} \cdot \boldsymbol{\eta}^{\epsilon} \mathrm{d} S, \quad \forall \boldsymbol{\eta}^{\epsilon} \in \mathbb{V}, \\
& \delta \mathfrak{F}^{\epsilon}\left(\chi_{n+1}, \boldsymbol{\tau}^{\epsilon}\right)=\int_{\Omega^{\epsilon}} \boldsymbol{\tau}^{\epsilon}:\left[\operatorname{sym} \nabla \boldsymbol{u}_{n+1}^{\epsilon}-\boldsymbol{\varepsilon}_{n+1}^{\epsilon}\right] \mathrm{d} \boldsymbol{x}=0, \quad \forall \boldsymbol{\tau}^{\epsilon} \in \mathbb{V}_{\sigma}, \\
& \delta \mathfrak{F}^{\epsilon}\left(\chi_{n+1}, \boldsymbol{r}^{\epsilon}\right)=\int_{\Omega^{\epsilon}} \boldsymbol{r}^{\epsilon}:\left\{\left[\nabla w_{n+1}^{\epsilon}-\boldsymbol{\sigma}_{n+1}^{\epsilon}\right]\right. \\
& \left.+\boldsymbol{C}_{n+1}^{\epsilon}:\left[\boldsymbol{\varepsilon}_{n+1}^{\mathrm{p} \epsilon}-\boldsymbol{\varepsilon}_{n}^{\mathrm{p} \epsilon}-\Delta \lambda^{\epsilon} \frac{\partial f_{n+1}^{\epsilon}}{\partial \boldsymbol{\sigma}_{n+1}^{\epsilon}}\right]\right\} \mathrm{d} \boldsymbol{x}=0, \forall \boldsymbol{r}^{\epsilon} \in \mathbb{V}_{\varepsilon}, \\
& \delta \mathfrak{F}^{\epsilon}\left(\chi_{n+1}, \boldsymbol{r}^{\mathrm{p} \epsilon}\right)=-\int_{\Omega^{\epsilon}} \boldsymbol{r}^{\mathrm{p} \epsilon}: \boldsymbol{C}_{n+1}^{\epsilon}:\left[\boldsymbol{\varepsilon}_{n+1}^{\mathrm{p} \epsilon}-\boldsymbol{\varepsilon}_{n}^{\mathrm{p} \epsilon}-\Delta \lambda^{\epsilon} \frac{\partial f_{n+1}^{\epsilon}}{\partial \boldsymbol{\sigma}_{n+1}^{\epsilon}}\right] \mathrm{d} \boldsymbol{x}=0, \quad \forall \boldsymbol{r}^{\mathrm{p} \epsilon} \in \mathbb{V}_{\varepsilon}, \\
& \delta \mathfrak{F}^{\epsilon}\left(\chi_{n+1}, p^{\epsilon}\right)=-\int_{\Omega^{\epsilon}} p^{\epsilon}\left[\left(D_{n+1}^{\epsilon}\right)^{-1}\left(q_{n+1}^{\epsilon}-q_{n}^{\epsilon}\right)-\Delta \lambda^{\epsilon} \frac{\partial f_{n+1}^{\epsilon}}{\partial q_{n+1}^{\epsilon}}\right] d x=0, \quad \forall p^{\epsilon} \in \mathbb{V}_{q}, \\
& \delta \mathfrak{F}^{\epsilon}\left(\chi_{n+1}, i^{\epsilon}\right)=\int_{\Omega^{\epsilon}} i^{\epsilon} f_{n+1}^{\epsilon} d x=0, \quad \forall i^{\epsilon} \in \mathbb{V}_{\lambda} .
\end{aligned}
$$

In the sequel of this subsection, we neglect for simplicity the iteration step indices. From the existence theory of linearized elasticity, we know that

$$
\dot{\boldsymbol{u}}^{\epsilon} \in\left[H^{1}\left(\Omega^{\epsilon}\right)\right]^{3}, \quad \dot{\boldsymbol{\varepsilon}}^{\epsilon} \in\left[L^{2}\left(\Omega^{\epsilon}\right)\right]^{6}, \quad \dot{\boldsymbol{\sigma}}^{\epsilon} \in\left[L^{2}\left(\Omega^{\epsilon}\right)\right]^{6}, \text { if } \dot{\sigma}_{i j}^{\epsilon}=\nabla w^{\epsilon}\left(\dot{\varepsilon}_{i j}^{\epsilon}\right) .
$$

We can show easily from the yield condition and the evolutionary law that

$$
\left\{\dot{\varepsilon}_{i j}^{\mathrm{p} \epsilon}, \dot{q}^{\epsilon}, q^{\epsilon}, \dot{\lambda}^{\epsilon}\right\} \in L^{2}\left(\Omega^{\epsilon}\right), \text { for every fixed } \Delta t .
$$

Indeed, from (110) 5 , we obtain that $\dot{\varepsilon}_{i j}^{\mathrm{e} \epsilon} \in L^{2}\left(\Omega^{\epsilon}\right)$. Then, from $(110)_{5},(110)_{1}$ and $(110)_{4}$, we obtain that $\dot{\varepsilon}_{i j}^{\mathrm{p} \epsilon} \in L^{2}\left(\Omega^{\epsilon}\right)$. We conclude that the functional setting introduced by the spaces $\mathbb{V}, \mathbb{V}_{\varepsilon}, \mathbb{V}_{\sigma}, \mathbb{V}_{q}, \mathbb{V}_{\lambda}$ is the natural setting for all admissible variations. Consequently, we can pass to the two-scale convergence limits [4] in the Euler-Lagrange Eqs. (122)-(127), since all functions are bounded in $L^{2}\left(\Omega^{\epsilon}\right)$.

7.2 An attempt to passing to the two-scale limits in the Euler-Lagrange equations: properties of the microinternal variables and the microevolutionary laws

In this subsection, by passing to the two-scale convergence limit in (122)-(127) we will obtain the global limiting equations satisfied by the microvariables. More specifically, following exactly the same procedure with Tsalis et al. [208], we can verify from (122) and (123) that the heterogeneous stress and strain converge to the microstress and microstrain defined in the first subsection of this section, related to the microdisplacement (55) and satisfying

$$
\boldsymbol{\sigma}_{n+1}^{\epsilon} \rightarrow_{t s} \boldsymbol{\sigma}_{n+1}^{0} \text { satisfying } \boldsymbol{\Sigma}_{n+1}=\frac{1}{|Y|} \int_{Y} \boldsymbol{\sigma}_{n+1}^{0}(\boldsymbol{y}) \mathrm{d} \boldsymbol{y},
$$

where $\rightarrow_{t s}$ denotes the two-scale convergence, and

$$
\boldsymbol{\varepsilon}_{n+1}^{\epsilon} \rightarrow_{t s} \boldsymbol{\varepsilon}_{n+1}^{0}=\operatorname{sym} \nabla_{x} \boldsymbol{u}^{0}+\operatorname{sym} \nabla_{\boldsymbol{y}} \boldsymbol{u}^{1}(\boldsymbol{x}, \boldsymbol{y}) \text { satisfying } \boldsymbol{E}=\frac{1}{|Y|} \int_{Y} \boldsymbol{\varepsilon}_{n+1}^{0}(\boldsymbol{y}) \mathrm{d} \boldsymbol{y} .
$$


We note that in (131) we used the two-scale convergence lemma for the gradient of a function [4]. We also note that the two-scale convergence of (122) gives the global (macro-) equation of equilibrium as well as the cell equation by a suitable choice of admissible variation functions [208].

From (124) and (125) choosing $\boldsymbol{r}^{\epsilon}=\boldsymbol{r}^{\mathrm{p} \epsilon}$,

$$
\int_{\Omega^{\epsilon}} \boldsymbol{r}^{\mathrm{p} \epsilon}: \frac{\partial w_{n+1}^{\mathrm{e} \epsilon}}{\partial \boldsymbol{\varepsilon}_{n+1}^{\mathrm{e} \epsilon}} \mathrm{d} \boldsymbol{x} \rightarrow t s \int_{\Omega} \int_{Y} \boldsymbol{r}^{\mathrm{p} 0}: \boldsymbol{\sigma}_{n+1}^{0} \mathrm{~d} \boldsymbol{y} \mathrm{d} \boldsymbol{x}=0, \quad \boldsymbol{r}^{\mathrm{p} 0} \in \mathbb{V}_{\varepsilon}
$$

Therefore, the two-scale limit of the heterogeneous energy gradient is indeed the microstress,

$$
\frac{\partial w_{n+1}^{\mathrm{e} \epsilon}}{\partial \boldsymbol{\varepsilon}_{n+1}^{\mathrm{e} \epsilon}}=\boldsymbol{C}_{n+1}^{\mathrm{e} \epsilon}: \boldsymbol{\varepsilon}_{n+1}^{\mathrm{e} \epsilon} \rightarrow \boldsymbol{\sigma}_{n+1}^{0} .
$$

From (127),

$$
\int_{\Omega} \frac{1}{|Y|} \int_{Y} i^{0} f_{n+1}^{0} \mathrm{~d} \boldsymbol{y} \mathrm{d} \boldsymbol{x}=0 .
$$

Equation (134) shows the existence of a microyield function $f_{n+1}^{0}$. The form of this function cannot be deduced from the heterogeneous properties, since it is a nonlinear function (see (110) 1 ) and $\boldsymbol{\sigma}_{n+1}^{\varepsilon}, q_{n+1}^{\varepsilon}$ converge only weakly to $\sigma_{n+1}^{0}, q_{n+1}^{0}$. However, starting from an assumed microyield function in terms of the microvariables $\boldsymbol{\sigma}_{n+1}^{0}, q_{n+1}^{0}, \boldsymbol{\varepsilon}_{n+1}^{\mathrm{p} 0}$,

$$
f_{n+1}^{0}\left(\sigma_{n+1}^{0}, q_{n+1}^{0}\right)=\left\|\boldsymbol{s}_{n+1}^{0}\right\|+q_{n+1}^{0}-\sqrt{\frac{2}{3}}\left(\sigma_{Y}^{0}\right)_{n+1} \leq 0,
$$

yields (127). Similarly, we cannot obtain explicit homogenized forms from the nonlinear (125) and (126), but only that an (unknown) function of $\dot{\boldsymbol{\varepsilon}}^{\mathrm{p} 0}$ and an (unknown) function of $\dot{q}^{p 0}$ are related to the microyield surface. However, a sufficient condition for the global dissipation is that the evolutionary equations for the microplastic strain rate and the microhardening, resulting from the microdissipation inequality as Euler-Lagrange equations, are satisfied at every point $y$ of the unit cell. The physical meaning of this mathematical conclusion is that the microevolutionary equations are precisely the Euler-Lagrange equations for the microdissipation inequality. In other words, the satisfaction of the microdissipation inequality is a sufficient condition for the satisfaction of the dissipation inequality of the heterogeneous body. We conclude that the microfunctions are divided in two categories. The first category comprises microdisplacement $\boldsymbol{u}^{0}$, microstress $\sigma^{0}$ and microstrain $\boldsymbol{\varepsilon}^{0}$, which are exactly the two-scale convergence limits of the heterogeneous Euler-Lagrange equations and are controlled directly by the macrostrain path. The second category, which comprises the set of discretized internal variables $\varepsilon^{\mathrm{p} 0}$ and $q^{0}$, is derived uniquely from the microdissipation inequality $d^{0} \geq 0$ for a choice of the microyield function $f^{0}$. Therefore, the combination of the two-scale convergence and the microdissipation leads to the proposed by Tsalis et al. [208] computational scheme.

\section{Exact mathematical homogenization of the elastoplastic model}

The fundamental assumptions for the homogenization of the generalized standard materials presented in Sects. 5 and 7 are (i) the existence of a global free energy which can be expressed as the mean value of the heterogeneous free energies and (ii) that, at the same time, the above function must be considered as a function of the internal microvariables [see (82)]. These assumptions permitted the application of Mandel-Hill's lemma and the mathematical treatment of the variation of the effective energy resulting to the definition of effective generalized forces [see (88)-(90)]. The first assumption is a consequence of the principle of additivity of energy. The same principle is assumed to hold for the dissipation [see (91)]. It is obvious that this is not a rigorous mathematical proof that the heterogeneous dissipation inequality, which gives the heterogeneous constitutive evolution of the internal parameters, converges to the effective dissipation inequality for the corresponding effective functions. This lack of rigorous mathematical homogenization will be compensated in this subsection, which is devoted to a more recent literature concerning this matter [66-68, 175, 183,213]. Concerning heterogeneous perfect elastoplasticity, Francfort and Giacomini [66] pointed out that the plastic strain rate $\dot{\boldsymbol{\varepsilon}}^{\mathrm{p}}$ is merely a measure, while the stress field $\boldsymbol{s}$ is typically not continuous, so that their product is not a 
priori meaningful. But that product plays an important role in the analysis of the problem; it also represents the mechanical dissipation. We recall that the mathematical homogenization consists in setting the heterogeneous problem, proving the existence of solutions independently of the heterogeneity parameter $\varepsilon$ and then passing to the limit for $\varepsilon \rightarrow \infty$ to obtain the homogenized problem. However, unless the problem is a problem of onedimensional plasticity [34-37], the limit problem involves an additional unknown, the microdisplacement [see for instance (55)], together with an additional partial differential equation, the microequilibrium equation, and it cannot give directly the effective evolution constitutive law, since upscaling (elimination of microvariable) is needed.

\subsection{On the slip lines in elastoplasticity}

Limit analysis is concerned with estimation of the collapse load in a direct (non-iterative) manner. The mechanism of deformation of a structure subjected to a gradually increasing load is the following: At the beginning, yield zones are still contained by elastic regions, but, as the loading is kept increasing, plastic zones communicate to create larger plastic regions becoming more and more important than the remaining elastic ones, whose role becomes more and more unimportant. The limit structure is a "mechanism" under collapse load, that is estimated bypassing the spreading phase of the contained plastic flow [42], allowing comparison with FEM elastoplastic solution which are difficult to perform when the boundaries of the plastic regions start to expand rapidly. Limit analysis is a theory applied to an ideal structure, at which, for constant loading, the deformation can increase illimitely, without hardening of the material and geometrical changes of the structure. Two known theorems, the upper- and the lower-bound theorems, furnish a range of values for the collapse load based respectively on a statically admissible stress field (satisfying equilibrium and yield conditions) and a kinematically admissible velocity field (satisfying kinematic compatibility and the flow rule associated with a yield criterion). The exact solution corresponds to all three requirements: equilibrium, compatibility, and constitutive relations. By a suitable choice of stress and velocity fields, the above range of values can be found as narrow as necessary for the particular structure and loading. In the application of the upper-bound theorem, one has to consider the rate of internal dissipation $\sigma: \dot{\boldsymbol{\varepsilon}}^{\mathrm{p}}$, where $\sigma$ satisfies the yield condition. Dissipation can be determined uniquely by a given plastic strain rate, contrarily to the stresses. When the plastic strain rate is continuous, expressions for different yield criteria are given as functions of the existing maximal engineering plastic strain rate and the uniaxial compression strength of the material (von Mises or Tresca criteria), or the principal compressive and tensile components of the plastic strain rate, the uniaxial compression and tension strengths and the internal friction of the material (Mohr-Coulomb criterium). In the context of the upper-bound theorem, it is assumed the existence of discontinuous velocities across moving internal surfaces (as distinct from fracture) or on the boundary of the structure, which idealize a continuous strain rate distribution with extremely rapid change across a thin transition layer. It is worth noticing that the dissipation occuring in this layer converges to a finite value as the thickness of the layer tends to zero [42]. In engineering applications, the discontinuity surface in Prandtl-Reuss or Tresca materials is the simplest one, across which the tangential velocity changes, separating the structure to two rigid blocks by a thin transition band, where the shear strain rate is uniform, and the occuring dissipation is approximated by the yield stress in simple shear multiplied by the relative velocity of the two blocks, independently of the thickness of the layer, that can be taken as small as one wishes. The above consequences of the mathematical theory of small-strain plasticity reveal the inconsistency of the above model, since velocity jumps cannot coexist with small-strain theories, as Tartar has repeatedly pointed out (see the discussion of Francfort and Giacomini [69]; for a number of questions related to homogenization and addressed to physicists by Tartar, see the introduction of his related work [201]), but it is still the most popular model.

\subsubsection{Approximating discontinuities}

It is now clear that the functional setting for the variational problems of perfect plasticity is more complicated than the functional setting of hardening materials, since (as obviously the setting of softening plasticity) it is a space $B D(\Omega)$. As mentioned before, in limit analysis discontinuities are allowed, either in displacements (strong discontinuities) or in strain (weak discontinuities) [88]. In mechanics-oriented words, strong discontinuities appear as slip lines, while weak discontinuities appear as shear bands. Shear bands act as precursor of material instabilities and since the end of the 80's are fully understood (see the review of Needleman and Tvergaard [152], as well as Wright [218] and Jirásek [97] for a useful objective modeling). Concerning strong 

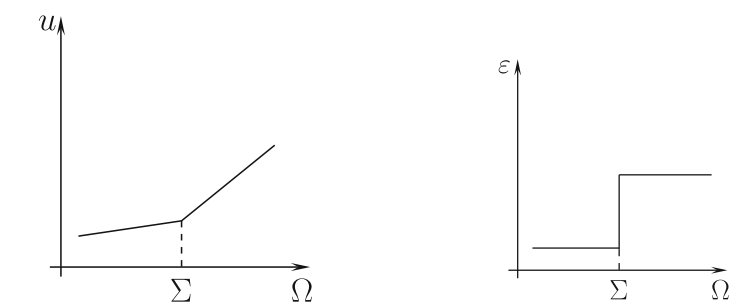

displacement field in weak discontinuity

strain field in weak discontinuity

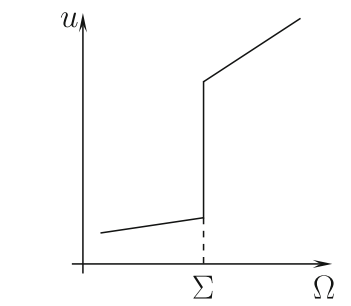

displacement field in strong discontinuity

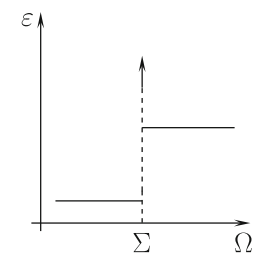

strain field in strong discontinuity

Fig. 1 Illustration of weak and strong discontinuities

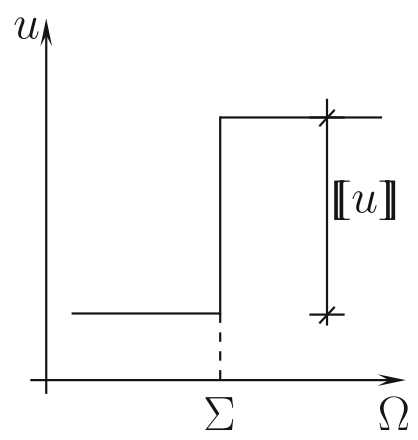

Fig. 2 Approximation of a function exhibiting a jump across the surface $\Sigma$

discontinuities induced by strain softening, Simo et al. [178] present an analysis based on the distributional interpretation of the softening law, which provides the perfectly plastic elastoplastic moduli, the associated acoustic tensor (independent of the softening modulus), the direction of the slip lines and the strength of the jump discontinuity. Moreover, the same authors verify these results by a regularizing finite element method in order to capture the functions of bounded variation without introducing viscosity parameters. Simo et al. [178], as well as Simo [179] (which extends these ideas to the finite strain deformation), base the proposed numerical approach on the decomposition of the functions of the motion into a regular and a discontinuous part, with the regular part satisfying the prescribed kinematic and static boundary conditions.

Continuum mechanics coexist with some discontinuous phenomena, such as slip lines in soils, shear bands in metals and cracks in rocks. The above phenomena are characterized by jumps in the displacement field which develop across fixed surfaces $\Sigma$, called material surfaces of discontinuity, as for instance the interfaces between highly different components in composites, and may act as precursor to failure. They have to be distinguished from weak discontinuities (jumps in the strain field, with continuous, non-smooth, displacement), developing along moving (non-material) surfaces, studied by various authors $[15,158,160,177,178]$, who presented a consistent theory in order to bridge the gap between continuum and discrete approaches to the strain localization phenomena that they called "strong discontinuities."

In Fig. 1, one-dimensional weak and strong discontinuities are depicted, together with the corresponding strains. In the first case, the strain exhibits a jump accross $\Sigma$, while in the second case the strain is a measure.

In Fig. 2, we see the approximation of a function exhibiting a jump across the surface $\Sigma$, in an onedimensional representation.

In Fig. $3_{a}$, we see the approximating function for $\delta$ very small, from which $\operatorname{tn} \omega=\frac{[|u|]}{\delta}$. In Fig. $3_{b}$, the gradient of the approximating function is depicted. 


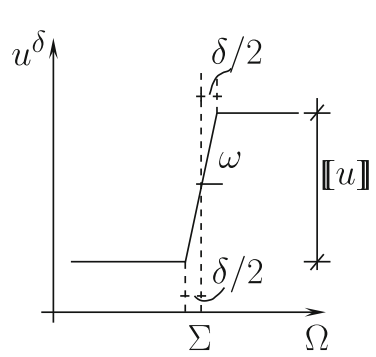

(a)

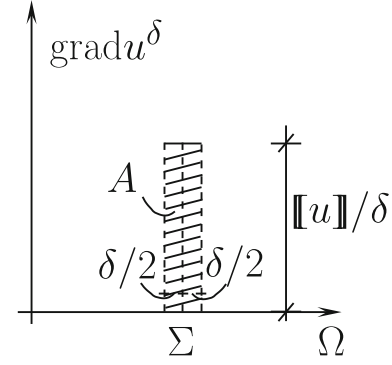

(b)

Fig. 3 a A function $u$ and $\mathbf{b}$ its gradient, when $u$ exhibits a jump across a surface $\Sigma$. The jump is approximated with the help of a small step $\delta$

We easily verify that the area $A$ remains, for every $\delta$, equal to the jump $[|u|]$. Recalling that the Heaviside function $H_{\Sigma}(\boldsymbol{x})$ is defined as $H_{\Sigma}(\boldsymbol{x})=1, \forall \boldsymbol{x} \in \Omega$ and $H_{\Sigma}(\boldsymbol{x})=0, \forall \boldsymbol{x} \in \Omega^{-}$, where $\Sigma$ splits the body $\Omega$ into two parts $\Omega^{+}$and $\Omega^{-}$with $\boldsymbol{n}$ being the unit normal vector pointing to $\Omega^{+}$, as well as that the Dirac's (line) delta function $\delta_{\Sigma}$ is defined by $\int_{\Omega} \delta_{\Sigma} \psi \mathrm{d} \Omega=\int_{\Sigma} \delta_{\Sigma} \psi d S, \forall \psi \in C_{0}^{\infty}(\Omega)$, the above approximations leads to the representation (viewed in the sense of distributions) of the displacement as

$$
\boldsymbol{u}(\boldsymbol{x}, t)=\boldsymbol{u}^{R}(\boldsymbol{x}, t)+H_{\Sigma}[|\boldsymbol{u}|](\boldsymbol{x}, t),
$$

where $[|\boldsymbol{u}|](\boldsymbol{x}, t)$ is a function describing the jumps, assumed to vary continuously everywhere in the body, while $\boldsymbol{u}^{R}(\boldsymbol{x}, t)$ is the regular part of the displacement. The assumption of continuously varying jumps can be omitted and is adopted here only for simplicity. Thus every jump of the displacement field is defined as

$$
\left.[|\boldsymbol{u}|](\boldsymbol{x}, t)\right|_{\boldsymbol{x} \in \Sigma}=[|\boldsymbol{u}|]_{\Sigma}
$$

and the corresponding strain is defined as

$$
\boldsymbol{\varepsilon}=\operatorname{sym}(\nabla \boldsymbol{u})=\operatorname{sym}\left(\nabla \boldsymbol{u}^{R}\right)+H_{\Sigma} \operatorname{sym}(\nabla[|\boldsymbol{u}|])+\delta_{\Sigma} \operatorname{sym}([|\boldsymbol{u}|] \otimes \boldsymbol{n}) .
$$

The last term is deduced from $\frac{\partial H_{\Sigma}}{\partial x_{i}}=n_{i} \delta_{\Sigma}$. It is worth noticing that the part $\overline{\boldsymbol{\varepsilon}}=\operatorname{sym}\left(\nabla \boldsymbol{u}^{R}\right)+H_{\Sigma} \operatorname{sym}(\nabla[|\boldsymbol{u}|])$ is absolutely continuous $(A C)$, while the part $\delta_{\Sigma} \operatorname{sym}([|\boldsymbol{u}|] \otimes \boldsymbol{n})$ is a singularly distributed part, a measure. In Sect. 8.3 a consistent mathematical theory concerning the functional setting and the existence theory of perfectly plastic materials is presented. It is also important to add that, in the case that the jump varies discontinuously in the body, the strain depends on an additional term which is discontinuous [137]. In the above reference, an excellent overview of the strong discontinuity approach (SDA) is given, where the fundamentals of the SDA are illustrated and compared to those of other, "discrete" models. Additionally, the numerical implementation of SDA is presented in an improved algorithmic framework using the return mapping algorithm.

\subsubsection{Discrete constitutive equation on a discontinuity surface with intrinsic softening parameter}

In various articles $[15,158-160,177,178]$, the SDA provides a discrete constitutive equation at the discontinuity path, which is consistent with the continuum constitutive equation, in such a way that the partial differential equations of the problem are well posed and the strain localization is allowed. The stress is assumed to be bounded everywhere in $\Omega$, the traction vector is assumed to be continuous across $\Sigma$ and the surface of discontinuity is assumed to be fixed with a normal $\boldsymbol{n}$ at any point, provided by the stress level at the initiation time.

The well posedness of the problem is ensured by the introduction of an intrinsic softening parameter of Dirac's delta structure (i.e., a measure), into the constitutive equation, making possible to regroup the bounded as well as the singular parts and deduce two separate constitutive laws. The intrinsic softening parameter is a material property related to the fracture energy of the bulk material. Since the softening parameter is a measure concentrated at the discontinuity surfaces, a perfect damage or perfect plasticity problem can be approached 
from a softening branch in the constitutive law. In this subsection, the above concept will be presented for the elastic-softening case (see the work by Oliver [159] for the general framework). The model is described by

$$
\begin{aligned}
\dot{\boldsymbol{\sigma}} & =\boldsymbol{C}:\left(\dot{\boldsymbol{\varepsilon}}-\dot{\boldsymbol{\varepsilon}}^{\mathrm{p}}\right), \\
\dot{\boldsymbol{\varepsilon}}^{\mathrm{p}} & =\dot{\lambda} \frac{\partial f}{\partial \boldsymbol{\sigma}}, \\
\dot{q} & =-\dot{\lambda} \mathcal{H} \frac{\partial f}{\partial q},
\end{aligned}
$$

where $f(\sigma, q)=\hat{f}(\sigma)+q-\sigma_{Y}, \dot{\lambda} \geq 0, f(\sigma, q) \leq 0, \dot{\lambda} f(\sigma, q)=0$, and where it is assumed that $\mathcal{H} \neq 0$ and

$$
\frac{1}{\mathcal{H}}=\frac{1}{\overline{\mathcal{H}}} \delta_{\Sigma}+\frac{1}{\mathcal{H}^{*}}
$$

It is obtained from $\dot{f}=0$ and (141) that

$$
\frac{1}{\mathcal{H}} \frac{\partial f}{\partial \sigma}: \dot{\sigma}=-\frac{1}{\mathcal{H}} \dot{q}=\dot{\lambda}
$$

hence, using (142),

$$
\dot{\lambda}=\frac{1}{\overline{\mathcal{H}}} \frac{\partial f}{\partial \boldsymbol{\sigma}}: \dot{\boldsymbol{\sigma}} \delta_{\Sigma}+\frac{1}{\mathcal{H}^{*}} \nabla f: \dot{\sigma}
$$

On the other hand, using again (141) and (142),

$$
\dot{\lambda}=\bar{\lambda} \delta_{\Sigma}+\lambda^{*}=-\frac{1}{\overline{\mathcal{H}}} \dot{q} \delta_{\Sigma}-\frac{1}{\mathcal{H}^{*}} \dot{q}
$$

The plastic multiplier can also be obtained from $\dot{f}=0$, the constitutive law (139) and (140) as

$$
\dot{\lambda}=\frac{\frac{\partial f}{\partial \boldsymbol{\sigma}}: \boldsymbol{C}: \dot{\boldsymbol{\varepsilon}}}{\mathcal{H}+\frac{\partial f}{\partial \boldsymbol{\sigma}}: \boldsymbol{C}: \frac{\partial f}{\partial \boldsymbol{\sigma}}} .
$$

Using again (139) and (140), we obtain the incremental constitutive law

$$
\dot{\sigma}=C^{\mathrm{ep}}: \dot{\varepsilon}
$$

where $C^{\text {ep }}$ is the elastoplastic tangent constitutive fourth-order tensor, whose element $i j k l$ is given by

$$
C_{i j k l}^{\mathrm{ep}}=C_{i j k l}-C_{i j p q} \frac{\partial f}{\partial \sigma_{p q}} \frac{\frac{\partial f}{\partial \sigma_{m n}} C_{m n k l}}{\mathcal{H}+\frac{\partial f}{\partial \sigma_{m n}} C_{m n p q} \frac{\partial f}{\partial \sigma_{p q}}} .
$$

Recalling again that $\mathcal{H} \neq 0$ and using (142),

$$
\dot{\boldsymbol{\varepsilon}}=\left(\boldsymbol{C}^{\mathrm{ep}}\right)^{-1}: \dot{\boldsymbol{\sigma}}=(\boldsymbol{C})^{-1}: \dot{\boldsymbol{\sigma}}+\frac{\delta_{\Sigma}}{\overline{\mathcal{H}}} \frac{\partial f}{\partial \boldsymbol{\sigma}} \otimes \frac{\partial f}{\partial \boldsymbol{\sigma}}: \dot{\boldsymbol{\sigma}}+\frac{1}{\mathcal{H}^{*}} \frac{\partial f}{\partial \boldsymbol{\sigma}} \otimes \frac{\partial f}{\partial \boldsymbol{\sigma}}: \dot{\boldsymbol{\sigma}} .
$$

We verify that the strain is divided into a bounded part

$$
\dot{\boldsymbol{\varepsilon}}^{\mathrm{e}}+\dot{\boldsymbol{\varepsilon}}^{\mathrm{p}}=\left(\boldsymbol{C}^{\mathrm{ep}}\right)^{-1}: \dot{\boldsymbol{\sigma}}=(\boldsymbol{C})^{-1}: \dot{\boldsymbol{\sigma}}+\frac{1}{\mathcal{H}^{*}} \frac{\partial f}{\partial \boldsymbol{\sigma}} \otimes \frac{\partial f}{\partial \boldsymbol{\sigma}}: \dot{\boldsymbol{\sigma}}
$$

and a singulary distributed part (a measure) of plastic nature

$$
\frac{\delta_{\Sigma}}{\overline{\mathcal{H}}} \frac{\partial f}{\partial \sigma} \otimes \frac{\partial f}{\partial \sigma}: \dot{\sigma} .
$$


Simo et al. [178] consider solutions for which $\lambda^{*}=0$ to show that

$$
\boldsymbol{Q}_{\Sigma} \cdot[|\dot{\boldsymbol{u}}|]=\bar{\lambda}\left(\boldsymbol{C}:\left[\frac{\partial f}{\partial \boldsymbol{\sigma}}\right]_{\Sigma}\right) \cdot \boldsymbol{n} \text { on } \Sigma,
$$

where $\boldsymbol{Q}_{\Sigma}:=\boldsymbol{n} \cdot \boldsymbol{C} \cdot \boldsymbol{n}$ is the elastic acoustic tensor at $\Sigma$.

For thermodynamical consistency reasons, the above procedure is restricted to the case of a softening material $\left(\mathcal{H}^{*}<0\right), \overline{\mathcal{H}}<0$ and is written here only formally in order to obtain the essence of the SDA. However, in Sect. 8.4.4, a complete mathematical treatment of the slip lines in perfect elastoplasticity will be presented.

Since the stress is smooth and the stress-like internal variable $q$ is a bounded function having at most jump discontinuities, the yield condition is valid under its classical form $f(\sigma, q) \leq 0$. Then, rate of yield function on $\Sigma$ yields to the regular part of plastic multiplier

$$
\bar{\lambda}=|[|\dot{\boldsymbol{u}}|]| \Xi, \quad \Xi:=\left[\frac{\left(\boldsymbol{C}: \frac{\partial f}{\partial \boldsymbol{\sigma}}\right): \boldsymbol{n} \otimes \boldsymbol{m}}{\frac{\partial f}{\partial \boldsymbol{\sigma}}: \boldsymbol{C}: \frac{\partial f}{\partial \boldsymbol{\sigma}}}\right]_{\Sigma}, \quad|\boldsymbol{m}|=1, \quad[|\dot{\boldsymbol{u}}|]=|[|\dot{\boldsymbol{u}}|]| \cdot \boldsymbol{m} .
$$

Then, recalling (152), the perfectly elastoplastic moduli on $\Sigma$ and the associated acoustic tensor, respectively, take the form

$$
(\boldsymbol{C})_{\Sigma}^{\mathrm{ep}}=\left[\boldsymbol{C}-\frac{\left(\boldsymbol{C}: \frac{\partial f}{\partial \boldsymbol{\sigma}}\right) \otimes\left(\boldsymbol{C}: \frac{\partial f}{\partial \boldsymbol{\sigma}}\right)}{\frac{\partial f}{\partial \boldsymbol{\sigma}}: \boldsymbol{C}: \frac{\partial f}{\partial \boldsymbol{\sigma}}}\right]_{\Sigma}, \quad \boldsymbol{Q}_{\Sigma}^{\mathrm{ep}}:=\boldsymbol{n} \cdot(\boldsymbol{C})_{\Sigma}^{\mathrm{ep}} \cdot \boldsymbol{n} \text { on } \Sigma .
$$

Combining (154) with (152) again gives the direction of the slip line $\boldsymbol{m}$,

$$
\boldsymbol{m}:=\frac{[|\dot{\boldsymbol{u}}|]}{|[|\dot{\boldsymbol{u}}|]|}, \quad \boldsymbol{Q}_{\Sigma}^{\mathrm{ep}} \cdot \boldsymbol{m}=\mathbf{0},
$$

and the strength of the jump discontinuity, which is uniquely defined by the softening modulus,

$$
|[|\dot{\boldsymbol{u}}|]|=\frac{1}{\Xi \bar{H}}\left[\left(\boldsymbol{C}: \frac{\partial f}{\partial \sigma}\right): \operatorname{sym} \nabla\right]_{\Sigma} .
$$

It is worth noticing that the direction of the slip line (155) is exactly the same in the perfectly plastic case [61].

The finite element method presented by Simo et al. [178] approximates the regular part of the displacement of the 1-D problem by continuous piecewise linear functions $N_{A}(\boldsymbol{x})$ as in the Galerkin method, and the singular part of the displacement by piecewise discontinuous functions with compact support $H_{A}(\boldsymbol{x})$. The displacement is considered in the form

$$
\boldsymbol{u}(\boldsymbol{x}, t)=\boldsymbol{u}(\boldsymbol{x}, \boldsymbol{t})^{R}+\sum_{i=1}^{m} H_{\xi_{i}}\left[\left|\boldsymbol{u}_{i}\right|\right],
$$

allowing for $\mathrm{m}$ jump discontinuities. The regular part is ideally approximated by continuous piecewise linear functions, while the singular interpolation part $H_{A}$ is regularized via a sequence of continuous functions $H_{A}^{k}$. For the next steps, including reparameterization of the displacement field in order to take into account the total boundary displacements, and the final interpolation in terms of relative nodal jumps, the reader is invited to see the work of Simo et al. [178].

Ebobisse and Reddy [61] presented a direct approach of the quasistatic primal problem in the bounded deformation and measures setting, without expanding the bilinear form

$$
a(\boldsymbol{w}(t), z)=\int_{\Omega}(\boldsymbol{\varepsilon}(\boldsymbol{v})-\boldsymbol{q}): \boldsymbol{C}:(\boldsymbol{\varepsilon}(\boldsymbol{u})-\boldsymbol{p}) \mathrm{d} \boldsymbol{x},
$$

since expansion reveals undesirable products of measures. The flow rule $\sigma \in \partial D$, for a dissipation $D$ corresponding to the von Mises criterion, is written in the form $s=c_{0} \frac{\dot{\boldsymbol{\varepsilon}}^{\mathrm{p}}}{\left|\dot{\boldsymbol{\varepsilon}}^{\mathrm{p}}\right|}, c_{0}=\sqrt{\frac{2}{3}} \sigma_{Y}$ and then the solution of 

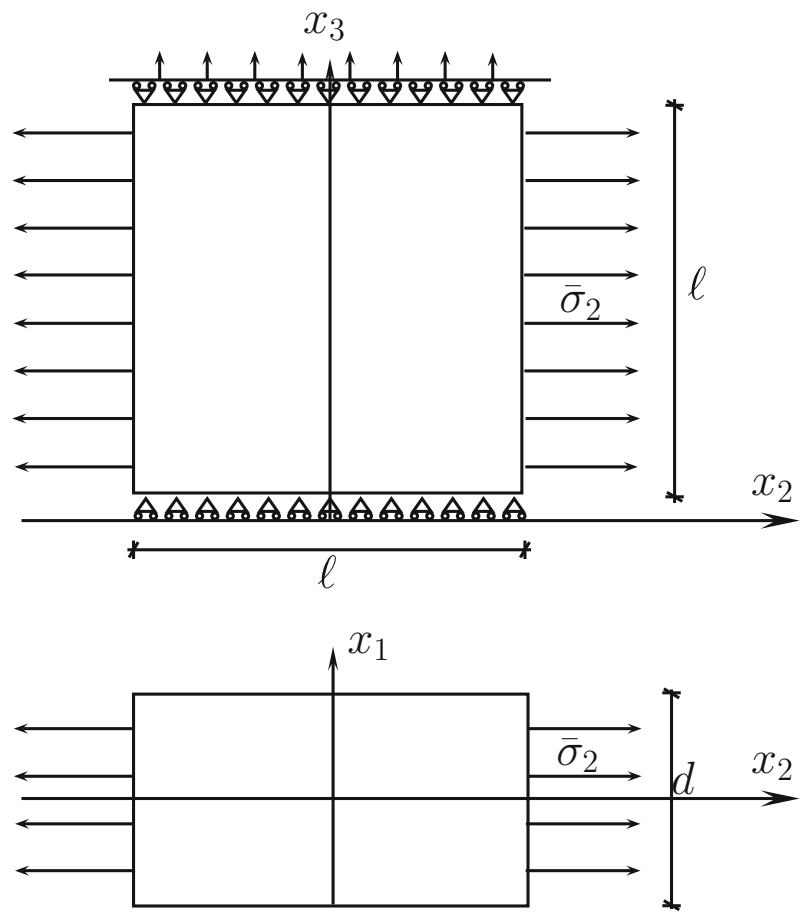

Fig. 4 Schematic representation of a biaxial test [72]

the primal problem is obtained as a weak limit of an interpolated solution of discretized problems. Denoting by $\Delta w_{n}$ the backward difference of $w$, the discretized flow rule for the step $n$ is written

$$
\boldsymbol{s}_{n}=c_{0} \frac{\Delta \boldsymbol{\varepsilon}_{n}^{\mathrm{p}}}{\left|\Delta \boldsymbol{\varepsilon}_{n}^{\mathrm{p}}\right|}=c_{0} \frac{\boldsymbol{\gamma}_{n}}{\left|\boldsymbol{\gamma}_{n}\right|}, \quad \boldsymbol{\gamma}_{n}:=\boldsymbol{e}\left(u_{n}\right)-\boldsymbol{\varepsilon}_{n-1}^{\mathrm{p}},
$$

for the absolutely continuous part of the plastic deformation. Due to the presence of time derivative, the nonlinear relation $s=c_{0} \frac{\dot{\varepsilon}^{\mathrm{p} a}}{\left|\dot{\boldsymbol{\varepsilon}}^{\mathrm{p} a}\right|}$ does not hold in the form $s \in \partial H\left(\dot{\boldsymbol{\varepsilon}}^{\mathrm{p} a}\right)$. The complete mathematical analysis of this problem is presented by Francfort and Giacomini [66].

\subsubsection{On the uniqueness of elastic-perfectly plastic processes}

In elastic-perfectly plastic evolutions, there exists uniqueness of the stress in the one-dimensional case. In the work by Kalamkarov et al. [102] uniqueness under a very specific loading is proven. In the two- or threedimensional setting no examples of uniqueness can be found, except in the case study of Francfort et al. [72]. In that paper, the authors prove uniqueness in the three-dimensional case assuming a set of boundary conditions which is different from the assumptions of the theory of Dal Maso et al. [47] presented in Sect. 3. More specifically, a set of mixed type boundary conditions is assumed, for which on part $S_{u}$ of the boundary the normal displacement $\boldsymbol{u} \cdot \boldsymbol{n}$ is controlled, while the tangential traction $(\boldsymbol{\sigma} \cdot \boldsymbol{n})_{\tau}$ remains nul. The above case is visualized by a biaxial test of a rectangular prismatic bar produced by tensile traction $\bar{\sigma}_{2}$ following $x_{2}$-axis, whose lateral sides are allowed to slip along $x_{2}$-axis without friction and have a known displacement along $x_{3}$-axis (Fig. 4).

For a von Mises material, in which $K=\left\{\boldsymbol{\tau} \in D_{\mathrm{sym}}^{3 \times 3}:|\boldsymbol{\tau}| \leq \sqrt{\frac{2}{3}} \sigma_{Y}\right\}$ and $H(\boldsymbol{p})=\sqrt{\frac{2}{3}} \sigma_{Y}|\boldsymbol{p}|, \boldsymbol{p} \in D_{\mathrm{sym}}^{3 \times 3}$, the uniqueness depends on the interplay between the yield stress $\sigma_{Y}$ and the applied stress $\bar{\sigma}_{2}$. Let us see in detail the results of Francfort et al. [72]. We note that the principal lines of the theory of the variational quasistatic evolution for heterogeneous materials proposed by Francfort and Giacomini [66] will be presented in Sect. 8.3. For a $S_{u}$ of class $C^{2}$, Francfort et al. [72] set $\dot{\boldsymbol{\varepsilon}}^{\mathrm{p}}(t)=\dot{\boldsymbol{\varepsilon}}^{\mathrm{p} a}(t)+\dot{\boldsymbol{\varepsilon}}^{\mathrm{ps}}(t)$. Then the flow rule is described by the equations: 
Flow rule for absolutely continuous plastic strain rate:

or

$$
\frac{\dot{\boldsymbol{\varepsilon}}^{\mathrm{p} a}(t, \boldsymbol{x})}{\left|\dot{\boldsymbol{\varepsilon}}^{\mathrm{p} a}(t, \boldsymbol{x})\right|} \in N_{K}(\boldsymbol{s}(t, \boldsymbol{x})), \text { for } \mathfrak{L}^{3}-\text { a.e. } \boldsymbol{x} \in\left\{\left|\dot{\boldsymbol{\varepsilon}}^{\mathrm{p} a}(t)\right|>0\right\},
$$

$$
|\boldsymbol{s}(t, \boldsymbol{x})|=\sqrt{\frac{2}{3}} \sigma_{Y} \text { and } \frac{\dot{\boldsymbol{\varepsilon}}^{\mathrm{p} a}(t, \boldsymbol{x})}{\left|\dot{\boldsymbol{\varepsilon}}^{\mathrm{p} a}(t, \boldsymbol{x})\right|}=\frac{\boldsymbol{s}(t, \boldsymbol{x})}{|\boldsymbol{s}(t, \boldsymbol{x})|}, \text { for } \mathfrak{L}^{3}-\text { a.e. } \boldsymbol{x} \in\left\{\left|\dot{\boldsymbol{\varepsilon}}^{\mathrm{p} a}(t)\right|>0\right\} .
$$

Boundary flow rule:

$$
\begin{gathered}
\left|[s(t) \cdot \boldsymbol{n}]_{\tau}(\boldsymbol{x})\right|=\sqrt{\frac{1}{3}} \sigma_{Y}, \\
\frac{[s(t) \cdot \boldsymbol{n}]_{\tau}(\boldsymbol{x})}{\left|[\boldsymbol{s}(t) \cdot \boldsymbol{n}]_{\tau}(\boldsymbol{x})\right|}=\frac{\dot{\overline{\boldsymbol{u}}}(t, \boldsymbol{x})-\dot{\boldsymbol{u}}(t, \boldsymbol{x})}{|\dot{\overrightarrow{\boldsymbol{u}}}(t, \boldsymbol{x})-\dot{\boldsymbol{u}}(t, \boldsymbol{x})|}, \text { for } \mathfrak{H}^{2}-\text { a.e. } \boldsymbol{x} \text { such that }|\dot{\overline{\boldsymbol{u}}}(t, \boldsymbol{x})-\dot{\boldsymbol{u}}(t, \boldsymbol{x})|>0, \\
\dot{\overline{\boldsymbol{u}}}(t, \boldsymbol{x})=\dot{\boldsymbol{u}}(t, \boldsymbol{x}), \text { for } \mathfrak{H}^{2}-\text { a.e. } \boldsymbol{x} \in\left\{\left|[s(t) \cdot \boldsymbol{n}]_{\tau}(\boldsymbol{x})\right|<\sqrt{\frac{1}{3}} \sigma_{Y}\right\} .
\end{gathered}
$$

Flow rule on the singular support of $\dot{\boldsymbol{\varepsilon}}^{\mathrm{p}}(t)$ :

$$
|\hat{\boldsymbol{s}}(t, \boldsymbol{x})|=\sqrt{\frac{2}{3}} \sigma_{Y} \text { and } \frac{\dot{\boldsymbol{\varepsilon}}^{\mathrm{p} s}(t)}{\left|\dot{\boldsymbol{\varepsilon}}^{\mathrm{p} s}(t)\right|}=\frac{\hat{\boldsymbol{s}}(t, \boldsymbol{x})}{|\hat{\boldsymbol{s}}(t, \boldsymbol{x})|}, \text { for }\left|\dot{\boldsymbol{\varepsilon}}^{\mathrm{p} s}(t)\right|-\text { a.e. } \boldsymbol{x} \in \Omega,
$$

where $\boldsymbol{s}^{r} \rightarrow \hat{\boldsymbol{s}}^{r}$ strongly in $L_{\left|\dot{\varepsilon}^{\mathrm{ps}}(t)\right|}^{1}\left(\Omega ; D_{\text {sym }}^{3}\right)$ and $\boldsymbol{\sigma}^{r}$ is the deviatoric part of stress average [66]

$$
\boldsymbol{\sigma}^{r}(t, \boldsymbol{x})=\frac{1}{\left|B_{r}(\boldsymbol{x}) \cap \Omega\right|} \int_{B_{r}(\boldsymbol{x}) \cap \Omega} \boldsymbol{\sigma}(t, \boldsymbol{z}) \mathrm{d} \boldsymbol{z}, \quad \forall r>0, \quad \boldsymbol{x} \in \Omega .
$$

As mentioned, the uniqueness for the stress depends on the relation between the applied stress $\bar{\sigma}_{2}$ and the yield stress $\sigma_{Y}$. Namely: (i) If $\bar{\sigma}_{2} \neq \frac{\sigma_{Y}}{\sqrt{3}}$, then there exists a unique quasistatic evolution, the homogeneous elastic-perfectly plastic evolution defined dy equations (3.8) - -(3.10), (3.13), (3.14) relative to the initial conditions (3.4), (3.1) of Section 3 in the paper of Francfort et al. [72]. (ii) If $\bar{\sigma}_{2}=\frac{\sigma_{Y}}{\sqrt{3}}$ the solution is no more the homogeneous solution of the previous case. The displacement exhibits a jump $\left(t-t_{c}\right) l\left(f_{1}+f_{3}\right)$ across the plane $\Gamma=\left\{x_{3}-x_{1}\right\}=\frac{l}{2}$, where $f_{i}, i=1,2,3$ are the unit vectors along the axis $x_{i}, i=1,2,3$. The plastic strain rate on $\Gamma$ is given by

$$
\dot{\boldsymbol{\varepsilon}}^{\mathrm{p}}=\left(t-t_{c}\right) \frac{l}{\sqrt{2}}\left(-\boldsymbol{f}_{1} \otimes \boldsymbol{f}_{1}+\boldsymbol{f}_{3} \otimes \boldsymbol{f}_{3}\right) \mathfrak{H}^{2}\lfloor\Gamma \text { on } \Gamma,
$$

where $t_{c}=\frac{1}{2 E}\left((1-2 v) \bar{\sigma}_{2}+\sqrt{4 \sigma_{Y}^{2}-3 \bar{\sigma}_{2}^{2}}, u_{3}=t l\right.$ on $x_{3}=l, t$ a computation time. It is worth noticing that, since $d<l$, the slip surface $\Gamma$ can be translated vertically to infinite parallel surfaces $\Gamma_{a}=\left\{\boldsymbol{x}+a \boldsymbol{f}_{3}: \boldsymbol{x} \in \Gamma,|a|<\frac{l-d}{2}\right\}$, along which the plastic slip can also take place [72]. It is also proven that the plastic strain there is of the form [72]

$$
\boldsymbol{\varepsilon}^{\mathrm{p}}(t, \boldsymbol{x})=\eta(t)\left(-\boldsymbol{f}_{1} \otimes \boldsymbol{f}_{1}+\boldsymbol{f}_{3} \otimes \boldsymbol{f}_{3}\right), \quad \eta \in W^{1,1}\left([0, \infty) ; M_{1}^{+}(\Omega)\right),
$$

where $\eta$ is given by

$$
\eta(t)=\left(t-t_{c}\right)\left[\zeta_{-}\left(x_{1}-x_{3}\right)+\zeta_{+}\left(x_{1}+x_{3}\right)\right], \zeta_{-}, \zeta_{+} \in M^{+}(R)
$$

and $\zeta_{-}, \zeta_{+}$two-dimensional Radon measures defined by

$$
\begin{aligned}
& <\zeta_{-}\left(x_{1}-x_{3}\right), \psi>=\frac{1}{2} \int\left[\psi\left(\frac{u+v}{2}, \frac{-u+v}{2}\right) \mathrm{d} \zeta_{-}(u)\right] \mathrm{d} v, \psi \in C_{c}^{\infty}\left(\Re^{2}\right), \\
& \left.<\zeta_{+}\left(x_{1}+x_{3}\right), \psi>=\frac{1}{2} \int\left[\psi\left(\frac{u+v}{2}, \frac{u-v}{2}\right) \mathrm{d} \zeta_{+}(u)\right]\right] \mathrm{d} v, \psi \in C_{c}^{\infty}\left(\Re^{2}\right),
\end{aligned}
$$

which is a wave solution of Cantor type. 
8.2 On the criteria of shear banding in heterogeneous elastoplastic evolutions

In one-dimensional adiabatic thermoviscoplasticity, criteria for the emergence of shear bands are related to the structure of the partial differential equations of momentum and energy describing the process and coincide with the conditions under which strain or/and temperature blows-up as a consequence of instability at a finite time. These criteria involve two stabilizing factors (strain hardening and strain rate sensitivity) and one destabilizing factor, the thermal softening [211]. In perfect plasticity, the only evidence of such discontinuities appears as singular limit of numerically computed high strains, without being extracted from the equations of plastic flow themselves [71], in the exception of Halphen [78]. Halphen [78] proposes an ad hoc flow rule in the jumps similar to the bulk flow rule. This lack of mathematically consistent criteria for the formation of slip lines in von Mises heterogeneous plasticity is covered in Francfort et al. [72], where the authors recover the classical flow rule by establishing a theorem based on "rather delicate duality arguments," and a boundary flow rule, originally derived in an earlier paper [66], which is a new ingredient in the modern formulation of elastoplasticity. Francfort et al. [71] proved for the first time a unified criterium for the possible stresses along the slip surfaces and for their directions, as a consequence of the proof of an additional flow rule acting on these surfaces. It is worth noticing that this criterium is the same with the criterium intuitively proposed by Halphen [78]. It allows for formulating a general result that may impose the absence of any plastic slip during a time interval of the quasistatic evolution, provided that the load and the initial conditions are sufficiently regular. This natural outcome of the variational evolution agrees with experiment evidence of shear banding, for which boundary and initial conditions are crucial. In this subsection we present the principal results of Francfort et al. [71], by recalling that details of the generic method of Francfort and Giacomini [66] will be presented in Sect. 8.3.

The Cauchy stress must satisfy the balance equations $-\operatorname{div} \boldsymbol{\sigma}(t)=\boldsymbol{b}(t)$, in $\Omega, \boldsymbol{\sigma}(t) \cdot \boldsymbol{n}=\boldsymbol{t}(t)$ on $S_{t}$ and the yield condition $|s(t, x)| \leq \sqrt{\frac{2}{3}} \sigma_{Y}$ for a.e. $\boldsymbol{x} \in \Omega$. The plastic flow is conditioned by the equality of dissipation rate and the plastic work $\sqrt{\frac{2}{3}} \sigma_{Y}\left|\dot{\boldsymbol{\varepsilon}}^{\mathrm{p}}\right|=<\boldsymbol{s}(t), \dot{\boldsymbol{\varepsilon}}^{\mathrm{p}}>$ as measures on $\Omega \cup S_{u}$, where, as defined, $<\boldsymbol{s}(t), \dot{\boldsymbol{\varepsilon}}^{\mathrm{p}}>$ denotes the duality between deviatoric stress and plastic strain rate explained by Francfort and Giacomini [66]. Then the absolutely continuous part of the plastic strain rate $\dot{\boldsymbol{\varepsilon}}^{\mathrm{p} a} \in L^{1}\left(D_{\mathrm{sym}}^{3 \times 3}\right)$ follows the flow rule (159) or (160), while

$$
\dot{\boldsymbol{\varepsilon}}^{\mathrm{p} a}(t, \boldsymbol{x})=0, \text { for } \mathfrak{L}^{3}-\text { a.e. } \boldsymbol{x} \in\left\{|\boldsymbol{s}(t)|<\sqrt{\frac{2}{3}} \sigma_{Y}\right\} .
$$

Moreover, the boundary tangential traction and the boundary displacement satisfy the flow rule (161)-(163). The singular part of plastic strain rate obeys to the flow rule (164). The new ingredient is the following flow rule for plastic slips [72]:

Flow rule on a slip surface $\Gamma_{\dot{u}(t)}$ The approximate stress $\hat{\boldsymbol{s}}(t)$ satisfies

$$
|\hat{\boldsymbol{s}}(t, \boldsymbol{x})|=\sqrt{\frac{2}{3}} \sigma_{Y} \text { and } \frac{[|\dot{\boldsymbol{u}}(t, \boldsymbol{x})|] \odot \boldsymbol{n}_{\dot{u}(t)}}{\mid[|\dot{\boldsymbol{u}}(t, \boldsymbol{x})|] \odot \boldsymbol{n}_{\dot{u}(t) \mid}}=\frac{\hat{\boldsymbol{s}}(t, \boldsymbol{x})}{|\hat{\boldsymbol{s}}(t, \boldsymbol{x})|} \text {, for } \mathfrak{H}^{2}-\text { a.e. } \boldsymbol{x} \in \Gamma_{\dot{u}(t)} .
$$

In particular, there exists a basis $\left(\boldsymbol{e}_{1}, \boldsymbol{e}_{2}, \dot{\boldsymbol{e}}_{3}\right)$ such that $\hat{\boldsymbol{s}}(t, \boldsymbol{x})=\operatorname{diag}\left(-\frac{\sigma_{Y}}{\sqrt{3}}, 0, \frac{\sigma_{Y}}{\sqrt{3}}\right)$. Moreover, the orthogonal lines determined by $\left(\dot{\boldsymbol{u}}(t, \boldsymbol{x}), \boldsymbol{n}_{\dot{u}(t, x)}\right)$ are bisected by $\left(\dot{\boldsymbol{e}}_{1}, \dot{\boldsymbol{e}}_{2}\right)$ (and vice versa). The authors use this result in order to formulate a condition which prevents the formation of slip surfaces:

Absence of plastic slips For an open $A \subseteq \Omega$, let the Cauchy stress satisfies (i) $\mathfrak{H}^{2}\left(S_{t}(t) \cap A\right)$ and (ii) the Lebesgue values $\tilde{\boldsymbol{\sigma}}(t, \boldsymbol{x})$ for $\boldsymbol{x} \in A$ do not satisfy $\hat{\boldsymbol{s}}(t, \boldsymbol{x})=\operatorname{diag}\left(-\frac{\sigma_{Y}}{\sqrt{3}}, 0, \frac{\sigma_{Y}}{\sqrt{3}}\right)$. Then, no plastic slip can occur on $A$ in the interval $\left[t_{1}, t_{2}\right]$, i.e., for every $t \in\left[t_{1}, t_{2}\right]$,

$$
[|\boldsymbol{u}(t)|] \odot \boldsymbol{n}_{u(t)} \mathfrak{H}^{2}\left\lfloor\left(\Gamma_{u(t)} \cap A\right)=[|\boldsymbol{u}(t)|] \odot \boldsymbol{n}_{u\left(t_{1}\right)} \mathfrak{H}^{2}\left\lfloor\left(\Gamma_{u\left(t_{1}\right)} \cap A\right) .\right.\right.
$$


In particular, if $\mathfrak{H}^{2}\left\lfloor\left(\Gamma_{u\left(t_{1}\right)} \cap A\right)=0\right.$, then for every $t \in\left[t_{1}, t_{2}\right]$,

$$
\boldsymbol{\varepsilon}^{\mathrm{p}}(t)=\boldsymbol{\varepsilon}^{\mathrm{p} a}(t) \mathfrak{L}^{3}+\boldsymbol{\varepsilon}^{c}(\boldsymbol{u}(t)) \text { on } A, \quad \boldsymbol{\varepsilon}^{\mathrm{p}}(t) \in L^{1}\left(\Omega ; D_{\text {sym }}^{3}\right) .
$$

As a consequence, if any tangential traction on $S_{u}$ is such that $\left|[s(t) \cdot n]_{\tau}\right|<\sqrt{\frac{1}{3}} \sigma_{Y}, \mathfrak{H}^{2}-$ a.e. on $A \cap_{u} S$, then no plastic slips occur on $A$ (boundary included) in $\left[t_{1}, t_{2}\right]$. The above condition of non-slip will be implied by suitable regularity on the Cauchy stress, namely by assuming that the body force and the initial stress are sufficiently regular, specifically $\boldsymbol{b} \in A C\left(0, T ; L^{2}\left(\Omega ; \Re^{3}\right)\right), \boldsymbol{t} \in A C\left(0, T ; L^{\infty}\left(S_{t} ; \Re^{3}\right)\right), D \boldsymbol{b} \in$ $\left.L^{\infty}\left(0, T ; L_{l o c}^{3}\left(\Omega ; M^{3 \times 3}\right)\right), \triangle \boldsymbol{b} \in L^{\infty}\left(0, T ; L_{l o c}^{3}\left(\Omega ; \Re^{3}\right)\right), \sigma_{0} \in H_{l o c}^{1}\left(\Omega ; M_{\text {sym }}^{3 \times 3}\right)\right)$. Then the stress satisfies for a.e. time in $(0, T)$ the regularity $\sigma \in L^{\infty}\left(0, T ; H_{l o c}^{1}\left(\Omega ; M_{\mathrm{sym}}^{3 \times 3}\right)\right)$, with Lebesgue points that have full $\mathfrak{H}^{2}-$ measure in $\Omega$. This ensures the following:

No-slip sufficient condition: Let $A \subseteq \Omega$ be an open set such that there exists $\eta>0$ with the principal deviatoric stresses $s^{1} \leq s^{2} \leq s^{3}$ satisfying $\left|s^{1}(t, \boldsymbol{x})+\frac{\sigma_{Y}}{\sqrt{3}}\right|+\left|s^{2}(t, \boldsymbol{x})\right|+\left|s^{3}(t, \boldsymbol{x})-\frac{\sigma_{Y}}{\sqrt{3}}\right| \geq \eta$ for a.e. $x \in A$ and a.e. $t \in\left[t_{1}, t_{2}\right]$. Then no plastic slip can occur on $A$ in $\left[t_{1}, t_{2}\right]$.

\subsection{Recent trends in heterogeneous elastoplastic evolutions}

The essential heterogeneity features are those concerning elasticity coefficients, yield surfaces and interfaces between material constituents. Solombrino [183] proposed the extension of the existence theory of Dal Maso et al. [47], presented in Sect. 3, to the heterogeneous case. In that paper, in order to overcome the difficulties posed by the presence of discontinuities in the space of plastically admissible stresses, only special (useful) cases of constituents are assumed, as that of where all phases are von Mises materials with different elasticity and yield functions. This case includes applications of great technological interest, such as bimetallic composites or composites made of fiber-reinforced matrix. However, pressure-dependent constituents, as polymers, are excluded. A more general consideration of the quasistatic problem of heterogeneous materials is presented by Francfort and Giacomini [66]. The first goal of that paper is the minimum requirements concerning the duality between stress and plastic strain, by relaxing the conditions assumed by Kohn and Temam [106] and revisited by Dal Maso et al. [47]. More specifically, it is shown in Sect. 6 of the work by Francfort and Giacomini [66] that a Lipschitz regularity of $S$ together with appropriate regularity on $\partial L_{S} S_{u}$ are sufficient to define a global (i.e., not separately in the interior of the domain and on its Dirichlet boundary) dissipation duality.

The proposed theory is founded on an existence theorem for a multi-component composite under the assumption that tangent stress to the interfaces, $[\boldsymbol{\sigma} \cdot \boldsymbol{n}]_{\tau}$, satisfies both plastically admissible sets $\left[\boldsymbol{K}_{i} \cdot \boldsymbol{n}\right]_{\tau}, i=$ 1,2 of the two components separated by this interface, where $\boldsymbol{K}_{i}$ are the admissible sets at $\boldsymbol{x} \in \Omega_{i}, i=1,2$. The authors remark that such a condition is known in limit analysis, citing the work of Suquet [190]. The authors emphasize the fact that, under this assumption, there is no need to consider the intersection of $\boldsymbol{K}_{i}$ on boundary, nor to impose an ordering of the admissible sets of different components. Then, the existence result of Solombrino [182] is recovered under Lipschitz assumption for the boundary:

Theorem: Existence result of a quasistatic evolution. Consider a geometrically admissible multi-component domain $\Omega$. Assume that the elastic coefficients and the continuous, convex and 1-homogeneous dissipation potential satisfy usual upper and lower bounds. Let the initial conditions $\left(\boldsymbol{u}_{0}, \boldsymbol{\varepsilon}_{0}, \boldsymbol{\varepsilon}_{0}^{\mathrm{p}}\right) \in \mathfrak{A}(\overline{\boldsymbol{u}}(0))$ fulfill the global stability condition (see Sect.3.3) $\mathfrak{Q}\left(\boldsymbol{\varepsilon}^{\mathrm{e}}(t)\right) \leq \mathfrak{Q}(\boldsymbol{\eta})+\mathfrak{R}\left(\boldsymbol{q}-\boldsymbol{\varepsilon}^{\mathrm{p}}(t)\right)$ for every elastic strain $\boldsymbol{\eta}$ and for every plastic strain $\boldsymbol{q}$, where the dissipation functional is defined by $\mathfrak{R}\left(\boldsymbol{\varepsilon}^{\mathrm{p}}\right):=\int_{\Omega \cup S_{u}} H\left(x, \frac{\boldsymbol{\varepsilon}^{\mathrm{p}}}{\mid \boldsymbol{\varepsilon}^{\mathrm{p} \mid}}\right) \mathrm{d}\left|\boldsymbol{\varepsilon}^{\mathrm{p}}\right|$ and the total elastic energy is defined by $\mathfrak{Q}\left(\varepsilon^{\mathrm{e}}\right)=\frac{1}{2} \int_{\Omega} \varepsilon^{\mathrm{e}}: C: \varepsilon^{\mathrm{e}}$. Then there exists a quasistatic evolution $\{t \mapsto$ $\left.\left(\boldsymbol{u}(t), \boldsymbol{\varepsilon}^{\mathrm{e}}(t), \boldsymbol{\varepsilon}^{\mathrm{p}}(t)\right), t \in[0, T]\right\}$ relative to the boundary displacement $\overline{\boldsymbol{u}}(t)$ such that $\left(\boldsymbol{u}(0), \boldsymbol{\varepsilon}^{\mathrm{e}}(0), \boldsymbol{\varepsilon}^{\mathrm{p}}(0)\right)=$ $\left(\boldsymbol{u}_{0}, \boldsymbol{\varepsilon}_{0}^{\mathrm{e}}, \boldsymbol{\varepsilon}_{0}^{\mathrm{p}}\right)$. Moreover, under the additional assumption that the interfaces can be approximated by piecewise $C^{2}$ surfaces, it is proven [66] that the above evolution satisfies equilibrium, boundary conditions as well as admissibility conditions of a classical evolution:

Theorem: Equilibrium and stress admissibility. Consider a $C^{2}$-geometrically admissible multi-component domain and an evolution $t \mapsto\left(\boldsymbol{u}(t), \boldsymbol{\varepsilon}^{\mathrm{e}}(t), \boldsymbol{\varepsilon}^{\mathrm{p}}(t)\right) \in \mathfrak{A}(\overline{\boldsymbol{u}}(t)$ satisfying the global stability inequality and the energy equality. Then, for every $t \in[0, T]$, the stress satisfies the balance equations and the plastic admissibility 
in the constituents. Moreover, along the inner interfaces $\Sigma$ and on the Dirichlet boundary $S_{u}$,

$$
\begin{gathered}
{[\boldsymbol{s} \cdot \boldsymbol{n}]_{\tau}(\boldsymbol{x}) \in K_{\Sigma}(\boldsymbol{x}), \text { for } \mathfrak{H}^{2}-\text { a.e. } \boldsymbol{x} \in \Sigma,} \\
\exists[\boldsymbol{s}(t) \cdot \boldsymbol{n}]_{\tau} \text { such that }[\boldsymbol{s}(t) \cdot \boldsymbol{n}]_{\tau} \in\left[\boldsymbol{K}_{i}(\boldsymbol{x}) \cdot \boldsymbol{n}(\boldsymbol{x})\right]_{\tau}, \text { for } \mathfrak{H}^{2}-\text { a.e. } \boldsymbol{x} \in \Sigma_{u} \cap \bar{\Omega}_{i},
\end{gathered}
$$

where $K_{\Sigma}(\boldsymbol{x}):=\left[\boldsymbol{K}_{i}(\boldsymbol{x}) \cdot \boldsymbol{n}(\boldsymbol{x})\right]_{\tau} \cap\left[\boldsymbol{K}_{j}(\boldsymbol{x}) \cdot \boldsymbol{n}(\boldsymbol{x})\right]_{\tau} \subseteq \Re^{3}$ and $\Sigma$ is an inner interface..

We note that the proof is based on a definition of a tangential stress on $\Delta \subset \partial A$, where $A$ a arbitrary Lipschitz subdomain of $\Omega$ with outer normal $\boldsymbol{n}$ such that $[\boldsymbol{\sigma} \cdot \boldsymbol{n}]_{\tau} \in L^{\infty}\left(\Delta ; \mathfrak{R}^{3}\right)$, with $\left\|[\boldsymbol{\sigma} \cdot \boldsymbol{n}]_{\tau}\right\|_{\infty} \leq\|\boldsymbol{s}\|_{\infty}$, and on a smooth approximation $\sigma_{k} \in C^{\infty}\left(\bar{A} ; M_{\mathrm{sym}}^{3}\right)$ of $\sigma_{k}$ satisfying

$$
\boldsymbol{\sigma}_{k} \rightarrow \boldsymbol{\sigma} \text { in } L^{2}\left(A ; M_{\mathrm{sym}}^{3}\right), \operatorname{div} \boldsymbol{\sigma}_{k} \rightarrow \operatorname{div} \boldsymbol{\sigma} \text { in } L^{2}\left(A ; \Re^{3}\right),\left\|\boldsymbol{s}_{k}\right\|_{\infty} \leq\|\boldsymbol{s}\|_{\infty} .
$$

The above admissibility constraint on the interfaces and on the Dirichlet boundary "... seems to be lacking in the existing literature" [66]. Emphasis is given by the authors on the different situation arising in homogenization [68].

Additionally, the classical flow rule is recovered in Sect. 3 of the work of Francfort and Giacomini [66], at the interfaces as well as on the Dirichlet boundary, "which seems to be a missing ingredient in the mechanics literature on elastoplasticity":

Theorem 8.1 Flow rule at the interfaces and on the Dirichlet boundary: For geometrically admissible multicomponent domain and admissible $\partial \operatorname{L}_{\partial S} S_{u}\left(\right.$ i.e., $\mathfrak{H}^{2}\left(\partial\left\llcorner_{\partial} S S_{u}\right)=0\right)$. Then, for a.e. $t \in[0, T]$,

$$
\begin{gathered}
\frac{\dot{\boldsymbol{\varepsilon}}^{p}(\boldsymbol{x})}{\left|\dot{\boldsymbol{\varepsilon}}^{p}(\boldsymbol{x})\right|} \in N_{K(\boldsymbol{x})}(\boldsymbol{s}(t, \boldsymbol{x})) \text { for a.e. } \boldsymbol{x} \in\left\{\left|\dot{\boldsymbol{\varepsilon}}^{p}(\boldsymbol{x})\right|>0,\right. \\
\frac{\dot{\boldsymbol{u}}^{i}(t, \boldsymbol{x})-\dot{\boldsymbol{u}}^{j}(t, \boldsymbol{x})}{\left|\dot{\boldsymbol{u}}^{i}(t, \boldsymbol{x})-\dot{\boldsymbol{u}}^{j}(t, \boldsymbol{x})\right|} \in N_{K_{\Sigma}(\boldsymbol{x})}\left([\boldsymbol{s}(t) \cdot \boldsymbol{n}]_{\tau}(\boldsymbol{x})\right) \text { for } \mathfrak{H}^{2} \text { a.e. } \boldsymbol{x} \in\left\{\left|\dot{\boldsymbol{u}}^{i}(t, \boldsymbol{x})-\dot{\boldsymbol{u}}^{j}(t, \boldsymbol{x})\right|>0\right\}
\end{gathered}
$$

where $\dot{\boldsymbol{u}}^{i}(t), \dot{\boldsymbol{u}}^{j}(t)$ are the traces on the interface $\Sigma_{i j}$ of the restrictions of $\dot{\boldsymbol{u}}(t)$ to the constituents $\Omega_{i}$ and $\Omega_{j}$, respectively, and $n$ points from $\Omega_{j}$ to $\Omega_{i}$, while $N_{K_{\Sigma}(\boldsymbol{x})}(\tau)$ denotes the normal cone to $K_{\Sigma}(\boldsymbol{x})$ at a tangent vector $\boldsymbol{\tau} \perp \boldsymbol{n}(\boldsymbol{x})$. Moreover, there exists a tangential stress $[\boldsymbol{s}(t) \cdot \boldsymbol{n})]_{\tau}$ such that, for $\mathfrak{H}^{2}$ a.e. $\boldsymbol{x} \in$ $S_{u} \cap \bar{\Omega}_{i}$ with $\left.\left|\dot{\bar{u}}(t, \boldsymbol{x})-\dot{\boldsymbol{u}}^{j}(t, \boldsymbol{x})\right|>0\right\}$,

$$
\frac{\dot{\overline{\boldsymbol{u}}}(t, \boldsymbol{x})-\dot{\boldsymbol{u}}(t, \boldsymbol{x})}{|\dot{\overline{\boldsymbol{u}}}(t, \boldsymbol{x})-\dot{\boldsymbol{u}}(t, \boldsymbol{x})|} \in N_{\left[\boldsymbol{K}_{i}(\boldsymbol{x}) \cdot \boldsymbol{n}(\boldsymbol{x})\right]_{\tau}}\left([s(t) \cdot \boldsymbol{n}]_{\tau}(\boldsymbol{x})\right) .
$$

Flow rule (180) holds for a well-chosen tangential trace of the traction along the Dirichlet boundary (see Theorem on stress admissibility on interfaces and Dirichlet boundary), which can be shown to be uniquely determined if $S_{u} \backslash \partial \Omega^{\prime}$ is of class $C^{2}$. Moreover, the authors emphasize the fact that, under strict convexity assumption on the set of admissible stresses in the constituents, a flow rule on the support of the singular part of $\dot{\boldsymbol{\varepsilon}}^{\mathrm{p}}$ in the constituents can be established. Numerical methods on elastoplasticity problems need to consider the above theorems concerning the admissibility and the flow rule on interfaces and boundary, under sufficient smoothness assumptions on the Dirichlet boundary, the interfaces, and the relative boundary of the Dirichlet boundary.

Finally, Hill's principle of maximum plastic work, adequately defined by the duality between stress and plastic strain, is recovered [66], revealing more information than the above flow rules, since it is not restricted to the Lebesgue-absolutely continuous part of $\dot{\varepsilon}$ :

Theorem: Hill's principle. Under admissible multi-component domain and relative boundaries of the Dirichlet boundary, the plastic work is maximal for a.e. $t \in[0, T]$, i.e.,

$$
<\boldsymbol{s}(t), \dot{\boldsymbol{\varepsilon}}^{\mathrm{p}}(t)>\left(\Omega \cup S_{u}\right)=\max \left\{<\zeta, \dot{\boldsymbol{\varepsilon}}^{\mathrm{p}}(t)>\left(\Omega \cup S_{u}\right): z \in \mathfrak{K}\right\}, z \text { deviatoric of } \zeta .
$$

It is worth noticing that it is proven [66] that introducing a vanishingly small (linear, isotropic) hardening in the above models yields to a plastic strain that cannot concentrate, so that the dissipation potential along interfaces is irrelevant and the limit coincide with the above elastoplastic evolution. 
8.4 Recent trends in homogenization of rate-dependent and rate-independent elastoplastic materials

\subsubsection{Von Mises or Tresca materials}

We first present the homogenization of von Mises or Tresca elastoplastic materials proposed by [213]. Here we will present the principal lines of the case of von Mises materials where it is recalled that $K$ stands for von Mises's yield criterium. For Tresca criterium the analysis follows similar steps. Using the subdifferential is necessary since $K$ may depend (and be highly oscillated) on $\boldsymbol{y}$. The pointwise evolution equation (76), resulting from the dissipation inequality (73) for every (generalized) force satisfying plastic criterium and involving the corresponding internal parameters, is now replaced by the inclusion

$$
\dot{\boldsymbol{\varepsilon}}^{\mathrm{p}} \equiv \frac{\partial \boldsymbol{\varepsilon}}{\partial t}-\boldsymbol{C}^{-1}: \frac{\partial \boldsymbol{\sigma}}{\partial t} \in \partial \chi_{K}(\boldsymbol{\sigma}),
$$

where $\chi_{K}$ is the indicator function for Von Mises yield criterium $K$.

Since plastic deformation is incompressible, this inclusion is equivalent to a linear elastic relation between stress and strain spheric components and the inclusion for the deviatoric strain rate

$$
\dot{\boldsymbol{e}} \in \partial \chi_{K}(\boldsymbol{s})\left(\subset D_{s}^{3 \times 3}\right) .
$$

By the definition of subdifferential, this also reads

$$
\dot{\boldsymbol{e}}:(\boldsymbol{s}-\boldsymbol{\xi}) \geq 0, \text { for } \boldsymbol{s} \in K \text { and for all } \xi \in \mathfrak{R}^{9} .
$$

Due to the plastic incompressibility, we recognize the plastic dissipation inequality, which forms the basis for the minimization problem described in Sect. 3.1.

The general case of viscous materials can be treated by a lower semicontinuous convex function $\varphi$, with inclusion

$$
\dot{\boldsymbol{\varepsilon}}^{\mathrm{p}} \equiv \frac{\partial \boldsymbol{\varepsilon}}{\partial t}-\boldsymbol{C}^{-1}: \frac{\partial \boldsymbol{\sigma}}{\partial t} \in \partial \varphi(\boldsymbol{\sigma}) .
$$

Using Fenchel's properties [64], Visintin formulated the dissipation inequality problem by the, equivalent to (185), inclusion

$$
\boldsymbol{\sigma}: \dot{\boldsymbol{\varepsilon}}^{\mathrm{p}} \in \partial \varphi(\boldsymbol{\sigma})+\partial \varphi^{*}\left(\dot{\boldsymbol{\varepsilon}}^{\mathrm{p}}\right),
$$

where $\varphi^{*}$ is the convex conjugate function of $\varphi$.

$$
\varphi^{*}: \Re^{N} \rightarrow \Re \cup\{+\infty\}: w \mapsto \sup \left\{\dot{\boldsymbol{\varepsilon}}^{\mathrm{p}}: \sigma-\varphi(\boldsymbol{\sigma}), \boldsymbol{\sigma} \in \Re^{N}\right\} .
$$

For von Mises perfect plasticity, $\varphi=\chi_{K}$ and

$$
\chi_{K}^{*}: K \rightarrow \Re \cup\{+\infty\}: \dot{\boldsymbol{\varepsilon}}^{\mathrm{p}} \mapsto \sup \left\{\dot{\boldsymbol{\varepsilon}}^{\mathrm{p}}: \sigma, \sigma \in K\right\} .
$$

In terms of total strain, (186) is equivalent to

$$
\boldsymbol{\sigma}: \dot{\boldsymbol{\varepsilon}}=\varphi(\boldsymbol{\sigma})+\varphi^{*}\left(\dot{\boldsymbol{\varepsilon}}-\boldsymbol{C}^{-1}: \dot{\boldsymbol{\sigma}}\right)+\frac{1}{2}\left(\boldsymbol{\sigma}: \boldsymbol{C}^{-1}: \boldsymbol{\sigma}\right),
$$

which is the pointwise power balance, and, due to Fenchel properties, also to the inequality

$$
\boldsymbol{\sigma}: \dot{\boldsymbol{\varepsilon}} \geq \varphi(\boldsymbol{\sigma})+\varphi^{*}\left(\dot{\boldsymbol{\varepsilon}}-\boldsymbol{C}^{-1}: \dot{\boldsymbol{\sigma}}\right)+\frac{1}{2}\left(\boldsymbol{\sigma}: \boldsymbol{C}^{-1}: \boldsymbol{\sigma}\right) .
$$

Visintin reformulated the minimization of dissipation problem as a null minimization problem:

$$
J(\sigma, \varepsilon)=\inf J=0,
$$

where

$$
J(\boldsymbol{\sigma}, \boldsymbol{\varepsilon}) \equiv \iint_{\Omega_{T}}\left[-\boldsymbol{\sigma}: \dot{\boldsymbol{\varepsilon}}+\varphi(\boldsymbol{\sigma})+\varphi^{*}\left(\dot{\boldsymbol{\varepsilon}}-\boldsymbol{C}^{-1}: \dot{\boldsymbol{\sigma}}\right)+\frac{1}{2}\left(\boldsymbol{\sigma}: \boldsymbol{C}^{-1}: \boldsymbol{\sigma}\right)\right] \mathrm{d} \boldsymbol{x} \mathrm{d} t
$$


Visintin [213] presented the weak formulation of an initial boundary value problem and an existence theorem for inhomogeneous viscoelastic materials exhibiting the evolution (182).

An extension of two-scale convergence to measures [12] is used in order to derive a two-length-scale model. Then, any dependence on the microvariable $y$ is eliminated in order to obtain a coarse-scale formulation, from which the constitutive relation turns to be non-local in time (memory effect), and it is not clear whether it might be rewritten as a gradient flow like the inclusion (185) in the unit cell. More specifically, it is shown an existence theorem for the limit functions $\left(\boldsymbol{u}^{0}, \boldsymbol{\varepsilon}^{0}, \sigma^{0}, \dot{\boldsymbol{\varepsilon}}^{\mathrm{p} 0}\right)$, which are obtained from the solution $\left(\boldsymbol{u}^{\epsilon}, \boldsymbol{\varepsilon}^{\epsilon}, \boldsymbol{\sigma}^{\epsilon}, \dot{\boldsymbol{\varepsilon}}^{\mathrm{p} \epsilon}\right)$ of the initial boundary value problem as $\epsilon \rightarrow 0$,

$$
\begin{gathered}
\boldsymbol{u}^{\epsilon} \rightarrow \boldsymbol{u}^{0} \text { weak star in }\left(C_{0}^{0}\left(\bar{\Omega}_{T} \times Y\right)^{3}\right)^{\prime}, \\
\boldsymbol{\varepsilon}^{\epsilon}=\operatorname{sym} \nabla \boldsymbol{u}^{\epsilon} \rightarrow_{t s} \boldsymbol{\varepsilon}^{0}=\operatorname{sym} \nabla_{\boldsymbol{x}} \boldsymbol{u}^{0}+\operatorname{sym} \nabla_{\boldsymbol{y}} \boldsymbol{u}^{1} \text { in }\left(C_{0}^{0}\left(\bar{\Omega}_{T} \times Y\right)^{3 \times 3}\right)^{\prime} \\
\boldsymbol{\sigma}^{\epsilon} \rightarrow_{t s} \boldsymbol{\sigma}^{0} \text { weak star in } L^{\infty}\left(\Omega_{T} \times Y\right)^{3 \times 3}, \\
\dot{\boldsymbol{\varepsilon}}^{\mathrm{p} \epsilon} \rightarrow_{t s} \dot{\boldsymbol{\varepsilon}}^{\mathrm{p} 0} \text { weak star in }\left(C_{0}^{0}\left(\bar{\Omega}_{T} \times Y\right)^{3 \times 3}\right)^{\prime} .
\end{gathered}
$$

where $\left(C_{0}^{0}\left(\overline{\Omega_{T}} \times Y\right)^{3}\right)^{\prime}$, or measures space, is the topological dual of the Banach space of continuous functions from the space-time domain to $\Re_{s}^{N}$ that vanish at the space-time boundary. We note the fact that the pointwise inclusion

$$
\dot{\boldsymbol{\varepsilon}}^{\mathrm{p} 0} \text { in } \partial \varphi(\boldsymbol{\sigma}, \boldsymbol{y}) \text { a.e. in } Y_{T}
$$

is equivalent to a global-in-time (integral) inequality, which is not clear if it can take the form of a gradient flow rule as in (197), and consequently it cannot allow to prove the existence of an effective constitutive evolution equation of the same form with the corresponding heterogeneous one.

\subsubsection{Prager model with kinematic hardening}

The situation seems to be more clear when the Prager model with kinematic hardening

$$
\dot{\boldsymbol{\varepsilon}}^{\mathrm{p}} \text { in } \partial \chi\left(\sigma-b \boldsymbol{\varepsilon}^{\mathrm{p}}\right), \quad b \geq \beta>0
$$

is studied [175]. The strict positivity of $b^{\epsilon}$ is crucial in deriving the energy estimates needed for the existence theorem (more specifically, in proving the $L^{2}\left(\Omega_{T}\right)$-boundness of $\dot{\boldsymbol{\varepsilon}}^{\mathrm{p} \epsilon}$ ). The initial boundary value problem describes the dynamic deformation, caused by initial displacement and stress, initial velocity, and initial plastic strain rate as internal variable, of a body subjected only to volume forces. From this point of view, this problem is useful since it corresponds to the most popular computational scheme in incremental elastoplasticity. Schweizer and Veneroni prove an existence theorem of strong solutions $\boldsymbol{u}^{\epsilon}, \boldsymbol{\varepsilon}^{\mathrm{p} \epsilon}, \boldsymbol{\sigma}^{\epsilon}$ of the sequence of problems $P^{\epsilon}$

$$
\begin{gathered}
\varrho^{\epsilon} \ddot{\boldsymbol{u}}^{\epsilon}-\operatorname{div} \boldsymbol{\sigma}^{\epsilon}=\boldsymbol{b} \\
\left(\boldsymbol{C}^{\epsilon}\right)^{-1}: \boldsymbol{\sigma}^{\epsilon}=\operatorname{sym} \nabla \boldsymbol{u}^{\epsilon}-\dot{\boldsymbol{\varepsilon}}^{\mathrm{p} \epsilon}, \\
\dot{\boldsymbol{\varepsilon}}^{\mathrm{p} \epsilon} \text { in } \partial \chi^{\epsilon}\left(\boldsymbol{\sigma}^{\epsilon}-b^{\epsilon} \boldsymbol{\varepsilon}^{\mathrm{p} \epsilon}\right),
\end{gathered}
$$

and an existence theorem of strong solution $\boldsymbol{u}^{0}: \Omega_{T} \rightarrow R^{n}, \boldsymbol{u}^{1}: \Omega_{T} \times Y \rightarrow R^{n}, \boldsymbol{\varepsilon}^{\mathrm{p} 0}, \boldsymbol{\sigma}^{0}: \Omega_{T} \times Y \rightarrow T_{S}^{3 \times 3}$ of the (homogenized) problem $P^{0}$

$$
\begin{gathered}
\varrho^{0} \ddot{\boldsymbol{u}}^{0}-\operatorname{div}_{\boldsymbol{x}}\left(\int_{Y} \boldsymbol{\sigma}^{0} \mathrm{~d} \boldsymbol{y}\right)=\boldsymbol{b} \\
(\boldsymbol{C}(\boldsymbol{y}))^{-1}: \boldsymbol{\sigma}^{0}=\operatorname{sym}_{\boldsymbol{x}} \boldsymbol{u}^{0}+\operatorname{sym} \nabla_{\boldsymbol{y}} \boldsymbol{u}^{1}-\dot{\boldsymbol{\varepsilon}}^{\mathrm{p} 0}, \\
\dot{\boldsymbol{\varepsilon}}^{\mathrm{p} 0} \text { in } \partial \chi\left(\sigma^{0}-b(\boldsymbol{y}) \boldsymbol{\varepsilon}^{\mathrm{p} 0} ; \boldsymbol{y}\right), \\
\operatorname{div}_{\boldsymbol{y}} \boldsymbol{\sigma}^{0}=\mathbf{0} .
\end{gathered}
$$

Then solutions of problem $P^{\epsilon}$ converge to solution of problem $P^{0}$ and

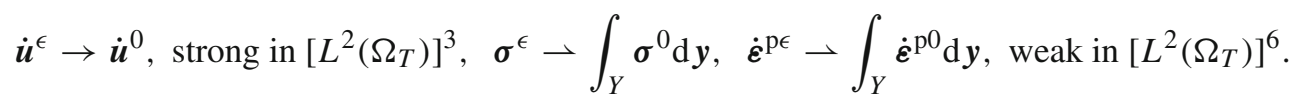

We note that the class of constitutive equations of monotone type described by Alber [3] includes the problem $P^{\epsilon}$ but not the limit problem $P^{0}$. 


\subsubsection{Elastoplasticity with hardening by energetic solutions}

The above regularizing effect of hardening is present in the works of Mielke and Timofte [139] and Visintin [213] too. Mielke and Timofte [139] treat the model of linearized elastoplasticity with hardening by using the notion of energetic solutions, "which is phrased in terms of a stability condition and an energy balance of an energy storage functional and a dissipation functional." In the sequel, we denote by $\boldsymbol{q}^{\epsilon}$ a heterogeneous energetic solution $\boldsymbol{q}^{\epsilon}=\left(\boldsymbol{u}^{\epsilon}, \boldsymbol{a}^{\epsilon}\right)$, i.e., a solution satisfying the stability and energy conditions (34) and (35) in Sect. 3.3, written in the form of the heterogenous problem $\left(S^{\epsilon}, E^{\epsilon}\right)$

$$
\begin{aligned}
\mathfrak{E}^{\epsilon}\left(t, \boldsymbol{u}^{\epsilon}(t), \boldsymbol{a}^{\epsilon}(t)\right) & \leq \mathfrak{E}^{\epsilon}(t, \tilde{\boldsymbol{u}}, \tilde{\boldsymbol{a}})+\mathfrak{R}^{\epsilon}\left(\tilde{\boldsymbol{a}}-\boldsymbol{a}^{\epsilon}(t)\right), \quad \forall \tilde{\boldsymbol{u}}, \tilde{\boldsymbol{a}} \\
\mathfrak{E}^{\epsilon}\left(t, \boldsymbol{u}^{\epsilon}, \boldsymbol{a}^{\epsilon}(t)\right)+\int_{0}^{t} \mathfrak{R}^{\epsilon}\left(\dot{\boldsymbol{a}}^{\epsilon}(s)\right) \mathrm{d} s & =\mathfrak{E}^{\epsilon}\left(0, \boldsymbol{u}^{\epsilon}(0), \boldsymbol{a}^{\epsilon}(0)\right)-\int_{0}^{t}<\dot{\boldsymbol{i}}(s) \mid \boldsymbol{u}(s)>\mathrm{d} s .
\end{aligned}
$$

The homogenized problem $(S, E)$ is defined by

$$
\begin{gathered}
\mathfrak{S}(t, \boldsymbol{U}(t), \boldsymbol{A}(t)) \leq \mathfrak{E}(t, \widetilde{\boldsymbol{U}}, \widetilde{\boldsymbol{A}})+\mathfrak{R}(\widetilde{\boldsymbol{A}}-\boldsymbol{A}(t)), \quad \forall \widetilde{\boldsymbol{U}}, \widetilde{\boldsymbol{A}} \\
\mathfrak{E}(t, \boldsymbol{U}(t), \boldsymbol{A}(t))+\int_{0}^{t} \mathfrak{R}\left(\dot{\boldsymbol{A}}^{\epsilon}(s)\right) \mathrm{d} s=\mathfrak{E}(0, \boldsymbol{U}(0), \boldsymbol{A}(0))-\int_{0}^{t}<\dot{\boldsymbol{l}}(s) \mid \boldsymbol{u}^{0}(s)>\mathrm{d} s .
\end{gathered}
$$

Mielke and Timofte [139] prove that the solutions $\left(\boldsymbol{u}^{\epsilon}, \boldsymbol{a}^{\epsilon}\right)$ of the $\epsilon$-periodic problem $\left(S^{\epsilon}, E^{\epsilon}\right)$ strongly two-scale cross-converge to a solution $(\boldsymbol{U}, \boldsymbol{A})$ of the two-scale homogenized problem $(S, E)$ under the sole assumption that the initial conditions strongly two-scale cross-converge, namely

$$
\begin{aligned}
\left(\boldsymbol{u}^{\epsilon}, \boldsymbol{a}^{\epsilon}\right) \rightarrow\left(\boldsymbol{u}^{0}, \boldsymbol{u}^{1}, \boldsymbol{A}\right) & \Rightarrow \mathfrak{E}\left(t, \boldsymbol{u}^{0}, \boldsymbol{u}^{1}, \boldsymbol{A}\right) \leq \lim _{\epsilon \rightarrow 0} \inf \mathfrak{E}^{\epsilon}\left(t, \boldsymbol{u}^{\epsilon}, \boldsymbol{a}^{\epsilon}\right) \text { and } \mathfrak{R}(\boldsymbol{A}) \leq \lim _{\epsilon \rightarrow 0} \inf \mathfrak{R}^{\epsilon}(\boldsymbol{a}(211) \\
& \forall\left(\boldsymbol{u}^{0}, \boldsymbol{u}^{1}, \boldsymbol{A}\right) \exists\left(\left(\boldsymbol{u}^{\epsilon}, \boldsymbol{a}^{\epsilon}\right)\right)^{\epsilon}:\left(\boldsymbol{u}^{\epsilon}, \boldsymbol{a}^{\epsilon}\right) \rightarrow\left(\boldsymbol{u}^{0}, \boldsymbol{u}^{1}, \boldsymbol{A}\right) \text { and } \\
& \mathfrak{E}^{\epsilon}\left(t, \boldsymbol{u}^{\epsilon}, \boldsymbol{a}^{\epsilon}\right) \rightarrow \mathfrak{E}\left(t, \boldsymbol{u}^{0}, \boldsymbol{u}^{1}, \boldsymbol{A}\right), \mathfrak{R}^{\epsilon}\left(\boldsymbol{a}^{\epsilon}\right) \rightarrow \mathfrak{R}(\boldsymbol{A}) .
\end{aligned}
$$

For the definition of cross-convergence, the reader is invited to consult [139]. Roughly speaking, the definition of weak (or strong) cross-convergence is equivalent to the weak (or strong) two-scale convergence of displacement, strain, and internal variables satisfying stability and energy conditions. There are three important conditions for the above convergence results: (i) the initial states are stable (including elastic equilibrium, (ii) the initial states converge in the two-scale sense and (iii) the initial energies converge. Under these conditions, if we start without any plasticity and the initial loading is small enough so that plasticity is not active, then (a) we have stability and (b) conditions (ii) and (iii) follow from classical static homogenization theory [136].

\subsubsection{Multi-phase perfectly elastoplastic composite}

Francfort and Giacomini [68] confront the homogenization of the evolution of a periodic multi-phase elastoplastic composite without any regularizing effect. In their paper, the authors "pay close attention to the issue of the duality between the stress fields which are essentially square-integrable functions and the plastic strains which are bounded measures; they attempt to clearly circumscribe those steps where duality is truly needed." The authors first investigate the consequences of the existence result (maximal dissipation, flow rule,...) for an evolution depended only on the microvariable, in order to use it for the homogenized problem, where both macro- and microstructural variables coexist, as we saw above. Moreover, they revisit the two-scale convergence of bounded measures in order to build the proper two-scale kinematics of elastoplasticity. Next, the authors address the homogenization elastoplastic process by first proving a lower semicontinuity result for the dissipation in a two-scale setting and an inequality between two-scale dissipation and two-scale plastic work. Then they prove that the heterogeneous problem defined in their previous work [66] two-scale converges at each time to a two-scale evolution on the two-scale limits $\boldsymbol{u}^{0}(t, \boldsymbol{x}), \boldsymbol{\varepsilon}^{\mathrm{e} 0}(t, \boldsymbol{x}, \boldsymbol{y})$ and $\boldsymbol{\varepsilon}^{\mathrm{p} 0}(t, \boldsymbol{x}, \boldsymbol{y})$ of the displacement, the elastic strain and the plastic strain, respectively. In the resulting equation, the dependence on the microstructural variable $\boldsymbol{y}$ cannot be integrated out, contrarily to the case of Visintin [213], which results to a thermodynamical model with an infinite number of internal variables, i.e., the plastic strains in the present case. This is a very important difference with the approximation of Suquet [193], as well as with the analysis of standard generalized materials described in Sect. 2.1, and with related computational techniques. Finally, the authors address the question of nature of the above homogenized behavior, namely of its constitutive evolution equation, and prove that, at almost every $\boldsymbol{x}$, the two-scale plastic flow follows the rule of normality, progressing normally to the $\boldsymbol{y}$-dependent yield surface. 


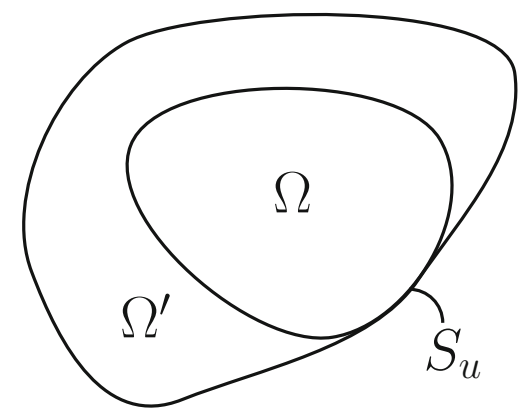

Fig. 5 Schematic representation of $\Omega, \Omega^{\prime}$ and $S_{u}$

For the reader's convenience, the principal lines of the above two-scale homogenization are presented in the sequel.

Hypotheses:

1. Force-free conditions.

2. The distribution

$$
\begin{aligned}
\left\langle\boldsymbol{s}, \boldsymbol{\varepsilon}^{\mathrm{p}}>(\psi):=\right. & \int_{\Omega} \psi \sigma_{i j}\left(\varepsilon_{i j}^{\mathrm{e}}(\boldsymbol{u})-\varepsilon_{i j}(\overline{\boldsymbol{u}})\right) \mathrm{d} \boldsymbol{x} \\
& -\frac{1}{2} \int_{\Omega} \sigma_{i j}\left[\left(u_{i}-\bar{u}_{i}\right) \frac{\partial \psi}{\partial x_{j}}+\left(u_{j}-\bar{u}_{j}\right) \frac{\partial \psi}{\partial x_{i}}\right] \mathrm{d} \boldsymbol{x}, \quad \forall \psi \in C_{c}^{\infty}\left(R^{3}\right)
\end{aligned}
$$

is a bounded Radon measure on $R^{3}$ with $\left|<s, \varepsilon^{\mathrm{p}}>\right| \leq\|\boldsymbol{s}\|_{\infty}\left|\boldsymbol{\varepsilon}^{\mathrm{p}}\right|$.

3. Usual assumptions on Hooke's law, coercivity of elasticity tensor, convexity of the plasticity criterium, positive one homogeneity, bounds, and interface properties of the dissipation potential.

4. Initial conditions $\left(\boldsymbol{u}_{0}^{\epsilon}, \varepsilon_{0}^{\mathrm{e} \epsilon}, \boldsymbol{\varepsilon}_{0}^{\mathrm{p} \epsilon}\right) \in A_{\epsilon}(\overline{\boldsymbol{u}}(0))$ for every $\epsilon$, globally stable configurations, satisfying, for every $\epsilon \rightarrow 0, \boldsymbol{u}_{0}^{\epsilon} \rightarrow \boldsymbol{u}_{0}$ weakly star in $B D\left(\Omega^{\prime}\right), \boldsymbol{\varepsilon}_{0}^{\mathrm{e} \epsilon} \rightarrow_{t s} \boldsymbol{\varepsilon}_{0}^{\mathrm{e}}$ two-scale strongly in $L^{2}\left(\Omega^{\prime} \times Y ; M_{\mathrm{sym}}^{3 \times 3}\right), \boldsymbol{\varepsilon}_{0}^{\mathrm{p} \epsilon} \rightarrow_{t s *} \varepsilon_{0}^{\mathrm{p}}$ two-scale weakly in $M_{1}\left(\Omega^{\prime} \times Y ; D^{3 \times 3}\right)$, where $\Omega^{\prime} \subset \Re^{3}$ open bounded set and such that $\Omega \subset \Omega^{\prime}$ and $\partial \Omega \cap \Omega^{\prime}=S_{u}$ (Fig. 5). Then we may extend $\left(\boldsymbol{u}, \boldsymbol{\varepsilon}^{\mathrm{e}}, \boldsymbol{\varepsilon}^{\mathrm{p}}\right) \in A(\overline{\boldsymbol{u}})$ to $\Omega^{\prime}$ by setting $\boldsymbol{u}=\overline{\boldsymbol{u}}, \boldsymbol{\varepsilon}^{\mathrm{e}}=\boldsymbol{\varepsilon}(\overline{\boldsymbol{u}}), \boldsymbol{\varepsilon}^{\mathrm{p}}=0$ on $\Omega^{\prime} \backslash \bar{\Omega}$.

Main result: Let $t \mapsto\left(\boldsymbol{u}^{\epsilon}(t), \boldsymbol{\varepsilon}^{\mathrm{e} \epsilon}(t), \boldsymbol{\varepsilon}^{\mathrm{p} \epsilon}(t)\right)$ be a quasistatic evolution relative to the boundary displacement $\overline{\boldsymbol{u}}(t)$ such that $\left(\boldsymbol{u}^{\epsilon}(0), \boldsymbol{\varepsilon}^{\mathrm{e} \epsilon}(0), \boldsymbol{\varepsilon}^{\mathrm{p} \epsilon}(0)\right)=\left(\boldsymbol{u}_{0}^{\epsilon}, \boldsymbol{\varepsilon}_{0}^{\mathrm{e} \epsilon}, \boldsymbol{\varepsilon}_{0}^{\mathrm{p} \epsilon}\right)$. Then there exists $\epsilon_{n} \rightarrow 0$ and a twoscale quasistatic evolution $t \mapsto\left(\boldsymbol{u}^{0}(t), \boldsymbol{\varepsilon}^{0}(t), \boldsymbol{\varepsilon}^{\mathrm{p} 0}(t)\right)$ relative to the boundary displacement $w(t)$ such that $\left(\boldsymbol{u}^{0}(0), \boldsymbol{\varepsilon}^{0}(0), \boldsymbol{\varepsilon}^{\mathrm{p} 0}(0)\right)=\left(\boldsymbol{u}_{0}, \boldsymbol{\varepsilon}_{0}^{\mathrm{e}}, \boldsymbol{\varepsilon}_{0}^{\mathrm{p}}\right)$ and such that, still denoting $\epsilon_{n} \rightarrow 0$ by $\epsilon \rightarrow 0$,

$$
\begin{gathered}
\boldsymbol{u}^{\epsilon}(t) \rightarrow_{*} \boldsymbol{u}^{0}(t) \text { weak star in } B D\left(\Omega^{\prime}\right), \\
\boldsymbol{\varepsilon}^{\mathrm{e} \epsilon}(t) \rightarrow_{t s} \boldsymbol{\varepsilon}^{\mathrm{e} 0}(t) \text { two scale weak in } L^{2}\left(\Omega^{\prime} \times Y ; M_{\mathrm{sym}}^{3 \times 3}\right), \\
\boldsymbol{\varepsilon}^{\mathrm{p} \epsilon}(t) \rightarrow_{t s *} \boldsymbol{\varepsilon}^{\mathrm{p} 0}(t) \text { two scale weak star in } M_{1}\left(\Omega^{\prime} \times Y ; D^{3 \times 3}\right),
\end{gathered}
$$

for every $t \in[0, T]$.

The following important mechanics-oriented conclusions are included [66]:

Stress admissibility (micro equilibrium problem) $[0, T]$

Let $t \mapsto\left(\boldsymbol{u}^{0}(t), \boldsymbol{\varepsilon}^{0}(t), \boldsymbol{\varepsilon}^{\mathrm{p} 0}(t)\right) \in A^{\text {hom }}(\overline{\boldsymbol{u}}(t))$ be a two-scale quasistatic evolution. Then, for every $t \in$

$$
\begin{aligned}
& \boldsymbol{\sigma}^{0}(t):=C: \boldsymbol{\varepsilon}^{\mathrm{e} 0}(t) \in K^{\mathrm{hom}} \\
& \boldsymbol{\sigma}^{0} \in L^{2}\left(\Omega \times Y ; M^{3 \times 3}\right)
\end{aligned}
$$

where $K^{\text {hom }}$ is the set of two-scale statically admissible stresses, satisfying the local equilibrium condition (cell problem)

$$
\operatorname{div}_{\boldsymbol{y}} \sigma^{0}=\mathbf{0} \text { on } \Omega \times Y, \quad \boldsymbol{s}^{0}(\boldsymbol{x}, \boldsymbol{y}) \in K(\boldsymbol{y}) \text { for } \mathfrak{L}_{\boldsymbol{x}}^{3} \otimes \mathfrak{L}_{\boldsymbol{y}}^{3}-\text { a.e. }(\boldsymbol{x}, \boldsymbol{y}) \in \Omega \times Y,
$$


and the global equilibrium condition

$$
\begin{aligned}
\operatorname{div}_{x} \boldsymbol{\Sigma} & =\mathbf{0} \text { on } \Omega, \boldsymbol{\Sigma} \cdot \boldsymbol{n}=\mathbf{0} \text { on } \partial \Omega \backslash \bar{S}_{u}, \\
\boldsymbol{\Sigma} & :=\int_{Y} \boldsymbol{\sigma}^{0}(\boldsymbol{x}, \boldsymbol{y}) \mathrm{d} \boldsymbol{y} .
\end{aligned}
$$

\section{Approximation of stress}

In order to define a disintegrated two-scale analogue of the duality measure $<\boldsymbol{s} \mid \boldsymbol{\varepsilon}^{\mathrm{p}}>$, Francfort and Giacomini [68] construct an adequate approximation of $\sigma^{0}$ in the following way: $\sigma^{0}$ is extended to $\Re^{3} \times Y$ by setting $\sigma^{0}=\mathbf{0}$ outside $\Omega$. For every $\boldsymbol{x} \in \partial \Omega$, an open neighborhood $U$ is considered such that $\partial \Omega \cap U$ is a Lipschitz subgraph. $\Omega$ is covered with open sets $U_{1}, \ldots, U_{m}$ associated with $x_{1}, \ldots, x_{m} \in \partial \Omega$. Assume there exists $\tau_{i} \in \mathfrak{R}^{3}$ such that $\left(U_{i} \cap \bar{\Omega}\right)+\alpha \tau_{i} \subset \subset \Omega, \quad 0<\alpha<1$. Let $\left\{\psi_{i}\right\}_{i=1}^{m}$ be a partition of unity of $\partial \Omega$ subordinated to $\left\{U_{i}\right\}_{i=1}^{m}$. The approximation by $\sigma_{n}^{0}$ of $\sigma^{0}$ is finally obtained by

$$
\boldsymbol{\sigma}_{n}^{0}=\rho_{n}(\boldsymbol{x}) \star\left[\sum_{i=1}^{m} \psi_{i}\left(\boldsymbol{x}+\alpha_{n} \tau_{i}\right) \boldsymbol{\sigma}^{0}\left(\boldsymbol{x}+\alpha_{n} \tau_{i}\right)+\left(1-\sum_{i=1}^{m} \psi_{i}(\boldsymbol{x})\right) \boldsymbol{\sigma}^{0}(\boldsymbol{x})\right],
$$

with $\alpha_{n} \searrow 0$ and $\left\{\rho_{n}\right\}, n \in N$ suitable convolution kernels.

Continuity in time

If the displacement $\boldsymbol{u}^{0}(t)$, the strain $\boldsymbol{\varepsilon}^{0}(t)$ and the plastic strain $\boldsymbol{\varepsilon}^{\mathrm{p} 0}(t)$ participate in a two-scale quasistatic evolution then $\left(\boldsymbol{u}^{0}, \boldsymbol{\varepsilon}^{0}, \boldsymbol{\varepsilon}^{\mathrm{p} 0}\right) \in A C\left(0, T ; B D\left(\Omega^{\prime}\right) \times L^{2}\left(\Omega^{\prime} \times Y ; M_{\mathrm{sym}}^{3 \times 3}\right) \times M_{1}\left(\Omega^{\prime} \times Y ; D^{3 \times 3}\right)\right)$. Moreover, velocity, strain rate and plastic strain rate $\left(\dot{\boldsymbol{u}}^{0}, \dot{\boldsymbol{\varepsilon}}^{0}, \dot{\boldsymbol{\varepsilon}}^{\mathrm{p} 0}\right)$ exist in the following sense

$$
\begin{aligned}
\dot{\boldsymbol{u}}^{0} & =\lim _{s \rightarrow t} \frac{\boldsymbol{u}(s)-\boldsymbol{u}(t)}{s-t} \text { weak star in } B D\left(\Omega^{\prime}\right), \\
\dot{\boldsymbol{\varepsilon}}^{0} & =\lim _{s \rightarrow t} \frac{\boldsymbol{\varepsilon}^{0}(s)-\boldsymbol{\varepsilon}^{0}(t)}{s-t} \text { strong in } L^{2}\left(\Omega^{\prime} \times Y ; M_{\mathrm{sym}}^{3 \times 3}\right), \\
\dot{\boldsymbol{\varepsilon}}^{\mathrm{p} 0} & =\lim _{s \rightarrow t} \frac{\boldsymbol{\varepsilon}^{\mathrm{p} 0}(s)-\boldsymbol{\varepsilon}^{\mathrm{p} 0}(t)}{s-t} \text { weak star in } M_{1}\left(\Omega^{\prime} \times Y ; D^{3 \times 3}\right),
\end{aligned}
$$

$\left(\dot{\boldsymbol{u}}^{0}, \dot{\boldsymbol{\varepsilon}}^{0}, \dot{\boldsymbol{\varepsilon}}^{\mathrm{p} 0}\right)$ being a two-scale quasistatic process for boundary displacement $\dot{\overline{\boldsymbol{u}}}^{0}$. Finally, the total dissipation in the body is given by a combination of micro- and macrovariables,

$$
\dot{\mathfrak{D}}^{\text {hom }}\left(0, t ; \boldsymbol{\varepsilon}^{\mathrm{p} 0}\right)=-\int_{\Omega \times Y} \boldsymbol{\sigma}^{0}(t): \dot{\boldsymbol{\varepsilon}}^{0}(t) \mathrm{d} \boldsymbol{x} \mathrm{d} \boldsymbol{y}+\int_{\Omega} \boldsymbol{\Sigma}(t): \boldsymbol{\varepsilon}(\dot{\overline{\boldsymbol{u}}}(t)) \mathrm{d} \boldsymbol{x} \in A C(0, T) .
$$

Explicit form of two-scale limit of strain

If $\boldsymbol{u}^{\epsilon} \vec{\sim}_{*} \boldsymbol{u}$ weak star in $B D(\Omega)$ and $\boldsymbol{\varepsilon}^{\epsilon} \rightarrow_{t . s .} \boldsymbol{\varepsilon}^{0}$ two-scale weak star in $M_{1}\left(\Omega^{\prime} \times Y ; M_{\mathrm{sym}}^{3 \times 3}\right)$, then there exists a measure $\boldsymbol{u}^{1} \in \mathfrak{X}(\Omega)$ (fluctuation of displacement) such that

$$
\boldsymbol{\varepsilon}^{0}=\operatorname{sym} \frac{\partial \boldsymbol{u}^{0}}{\partial \boldsymbol{x}} \otimes \mathfrak{L}_{\boldsymbol{y}}^{3}+\operatorname{sym} \frac{\partial \boldsymbol{u}^{1}}{\partial \boldsymbol{y}} .
$$

The remaining important question is the interpretation of the energy inequality of the two-scale quasistatic evolution in terms of a constitutive law. In homogeneous materials, this is given by the usual flow rule. Can one obtain an explicit constitutive law from the above homogenization process? How close to the flow rule of a consistent thermodynamical model is this?

Two-scale flow rule

For displacement, strain and plastic strain $\left(\boldsymbol{u}^{0}(t), \boldsymbol{\varepsilon}^{0}(t), \boldsymbol{\varepsilon}^{\mathrm{p} 0}(t)\right.$ satisfying the conditions of a two-scale quasistatic evolution, and for a.e. $t \in[0, T]$, the rates $\left(\dot{\boldsymbol{u}}^{0}(t), \dot{\boldsymbol{\varepsilon}}^{0}(t), \dot{\boldsymbol{\varepsilon}}^{\mathrm{p} 0}(t)\right.$ are also a two-scale quasistatic evolution and, for $\mathfrak{L}_{\boldsymbol{x}}^{3}$ - a.e. $\boldsymbol{x} \in \Omega$, the absolutely continuous part of the plastic strain rate is given by

$$
\frac{\left(\dot{\boldsymbol{\varepsilon}}^{\mathrm{p} 0}\right)_{x}(t)}{\left|\left(\dot{\boldsymbol{\varepsilon}}^{\mathrm{p} 0}\right)_{x}(t)\right|}(\boldsymbol{y}) \in N_{K(\boldsymbol{y})}\left(\boldsymbol{s}^{0}(t, \boldsymbol{x}, \boldsymbol{y})\right) \text { for } \mathfrak{L}_{\boldsymbol{y}}^{3}-\text { a.e. } \boldsymbol{y} \in\left\{\left|\left(\dot{\boldsymbol{\varepsilon}}^{\mathrm{p} 0}\right)_{x}(t)\right|>0\right\},
$$


where $\left(\dot{\boldsymbol{\varepsilon}}^{\mathrm{p} 0}\right)(t)=\boldsymbol{\eta} \otimes^{g e n}\left(\dot{\boldsymbol{\varepsilon}}^{\mathrm{p} 0}\right)_{x}(t)$ and $\boldsymbol{\eta}=\mathfrak{L}_{x}^{3}+\left(\operatorname{proj}_{\sharp}\left|\boldsymbol{\varepsilon}^{\mathrm{p} 0}\right|\right)^{s} \in M_{1}^{+}\left(\Omega^{\prime}\right)$. As is pointed out by Francfort and Giacomini [68], the continuity in time in the sense of (225) does not hold for $\left(\dot{\varepsilon}^{\mathrm{p} 0}\right)_{x}(t)$ due to the above disintegration of $\left(\dot{\varepsilon}^{\mathrm{p} 0}\right)_{x}(t)$ : "Consequently, the flow rule...cannot be construed as completely vindicating the two-scale evolution as that corresponding to a generalized standard material in the sense of Halphen and Nguyen [79]". Moreover, this flow rule is not a flow rule in $\boldsymbol{x}$ for a Lebesgue measure $\mathcal{L}_{y}^{3}-$ a.e. $\boldsymbol{y} \in Y$, as a flow rule of a consistent thermodynamical model.

Additionally, the flow rule on the interfaces, satisfied by the rate of fluctuations of the microdisplacement, is obtained in the form [68]

$$
\frac{\dot{\boldsymbol{u}}^{1 i}(t, \boldsymbol{y})_{x}-\dot{\boldsymbol{u}}^{1 j}(t, \boldsymbol{y})_{x}}{\left|\dot{\boldsymbol{u}}^{1 i}(t, \boldsymbol{y})_{x}-\dot{\boldsymbol{u}}^{1 j}(t, \boldsymbol{y})_{x}\right| \in N_{K_{\Gamma(\boldsymbol{y})}}}\left(\left[\boldsymbol{s}^{0}(t, \boldsymbol{x}) \cdot \boldsymbol{n}\right]_{\tau}(\boldsymbol{y})\right),
$$

for $\mathcal{H}^{2}$ - a.e. $\boldsymbol{y} \in\left\{\dot{\boldsymbol{u}}^{1 i} \neq \dot{\boldsymbol{u}}^{1 j}\right\}$, where $\dot{\boldsymbol{u}}^{1 i}, \dot{\boldsymbol{u}}^{1 j}$ are the traces on the interfaces $\Gamma_{i j}$ of the restrictions of the rate of microdisplacement fluctuations on $Y_{i}, Y_{j}$ respectively, $n$ points from $Y_{j}$ to $Y_{i}$ and $N_{K_{\Gamma(y)}}(\sigma)$ denotes the normal cone to $K_{\Gamma(\boldsymbol{y})}$ at a vector $\boldsymbol{\tau} \perp \boldsymbol{n}(\boldsymbol{y})$.

Recently, a generalized definition of the quasistatic evolution for linearly elastic-perfectly plastic materials, deduced from Hill's principle of maximum plastic work and interpreted as the requirement that, along the process, the stress-strain path is maximal dissipative, is presented by Solombrino [183] (see Mielke [138] for the related theory on energy dissipation balance). The dissipation measure is defined as the supremum of the dualities between the deviatoric parts of stress and plastic strain rates, without any continuity assumption on the yield stress. This allows for an abstract dual version of the Prandtl-Reuss evolution equation, resulting to the measure-theoretical version of Francfort and Giacomini [66], if the yield surface is piecewise continuous. This new formulation is equivalent to an (integral) energy dissipation balance, or a (differential) balance of powers, forming the basis of computational evolutionary problems in elastoplasticity. The existence of this process is proved via a vanishing viscosity approach and variational techniques from elastoplastic problems. The main mechanics-oriented contribution of this paper is that this formulation fits better with the nature of plastic deformation, for which the yield surfaces are generally spatially and time dependent, and it is not possible to consider a priori piecewise continuous spatial dependence.

\subsubsection{Recent trends on incremental methods}

Homogenization- or micromechanics-based computational methods have been proposed in order to define the overall behavior of viscoelastic, elastoplastic, viscoplastic or damaged composites, or fully coupled thermomechanical models (higher order theories [2], thermoviscoelasticity of rubber-like matrix composites [1], fully implicit formulation of elastoplastic composites [16], parametric finite-volume-based theories $[28,30,108]$. Recent homogenization papers present computational methods to investigate smart structures with high demands in strength, multifunctionality and durability, their thermomechanical behavior under fatigue [19], or their magnetomechanical [40,95] or thermomagnetoelectroelastic [22] response.

Computational homogenization of elastoplastic composites based on mathematical homogenization was the subject of many works under quite different points of view: Fish et al. [65] generalized the classical homogenization theory to account for eigenstrains expanded in double-scale expression too, and are related by close form to the mechanical fields in the constituents. The overall behavior is obtained by averaging the phase concentration factors. On the other hand, in the fully implicit formulation [16], the boundary value problem, consisting of the virtual work equation, a micro-macrokinematic relation and a linearized constitutive law, is fully implicitly formulated to compute incremental microdisplacement in the unit cell. The homogenization scheme is based on iterative solution with quadratic convergence by successively updating incremental strains. Initial incremental strain is crucial for the convergence. This method was rebuilt by introducing half unit cells to reduce computational costs and applied to honeycombs indented by a flat punch by a full-scale finite element analysis [17]. Recently, a computational homogenization scheme for heterogeneous shell structures was proposed on the basis of an adequate definition of the deformation fields using two-scale convergence theory [208]. The discrete variational formulation for both the micro- and macroscale is presented. The implicit formulation of the homogenization problem is proposed, together with the numerical scheme and the computation of the consistent tangent modulus. The computational algorithm consists of four interactive problems: the macroscale, the microscale, the iterative plastic scheme, and the effective tangent modulus problem.

Micro-to-macrotransitions are also presented in the context of homogenization of shape memory alloys $[39,80,109]$ on the basis of a thermomechanical constitutive model introduced by Qidwai and Lagoudas [173] using return mapping algorithm. 
Finally, modeling thermomechanical problems of heterogeneous materials meets the increased needs of many engineering applications in automotive and aerospace industry $[38,117,121,176]$.

Multiscale modeling of complex viscoplastic composites of technological importance, such as composites showing complicated failure mechanisms under fatigue tests or woven composites with plastic matrix exhibiting high temperature-sensitivity, needs a computational homogenization framework enriched by incremental plasticity techniques. A model for incremental plasticity-based homogenization can use all the results of linear quasistatic elasticity concerning the functional setting of solutions of the problem, namely the $H^{1}$-weak convergence of displacement and the $\mathcal{L}^{2}$-weak convergence of strain and stress, as well as the cell problem, provided that the elastic coefficients are replaced by the (path-dependent) tangent modulus, at both the microand the macroscale. Passing to the limit in the weak form of equilibrium equation gives that the incremental macrostress is the mean value of the microstress, and that it satisfies the macroequilibrium equation. On the other hand, passing to the limit in the heterogeneous virtual work equation and using the two-scale convergence properties for $H^{1}$-functions and their derivatives gives the expression of incremental macrostrain in terms of the incremental microdisplacemental gradient. We recall that the above findings are independent of the constitutive law, but in revenge they crucially depend on the functional setting. However, in the case of a fully coupled thermomechanical modeling, where the energy equation and the second law of thermodynamics are added to the heterogeneous system of partial differential equations, one more source of difficulties regarding the homogenization process is added too: The presence of time rates of internal and free energies in the energy and second law equations, as well as in the local dissipation. This involves products of oscillating functions whose homogenized expressions cannot take the form of products of homogenized quantities. In the thermomechanical case, the free energy studied in Sect. 5 (68) is of the form

$$
\psi^{\epsilon}=\psi^{\epsilon}\left(\boldsymbol{\varepsilon}^{\epsilon}, \theta^{\epsilon}, \boldsymbol{a}^{\epsilon}\right),
$$

in terms of strain, temperature $\theta$, and internal parameters. The energy equation and the second law of thermodynamics read, respectively,

$$
\begin{gathered}
\frac{\partial q_{i}^{\epsilon}}{\partial x_{i}}=r^{\epsilon}=\sigma_{i j}^{\epsilon} \dot{\varepsilon}_{i j}^{\epsilon}-\theta^{\epsilon} \dot{s}^{\epsilon}-\dot{\theta}^{\epsilon} s^{\epsilon}-\dot{\psi}^{\epsilon}, \\
\gamma^{\epsilon}-\frac{1}{\theta^{\epsilon}} q_{i}^{\epsilon} \frac{\partial \theta^{\epsilon}}{\partial x_{i}} \geq 0,
\end{gathered}
$$

being coupled by

$$
\gamma^{\epsilon}=\theta^{\epsilon} \dot{s}^{\epsilon}+r^{\epsilon}=-\frac{\partial \psi^{\epsilon}\left(\boldsymbol{\varepsilon}^{\epsilon}, \theta^{\epsilon}, \boldsymbol{a}^{\epsilon}\right)}{\partial \boldsymbol{a}^{\epsilon}}: \dot{\boldsymbol{a}}^{\epsilon}
$$

where $\gamma^{\epsilon}$ denotes the local heterogeneous dissipation due to mechanical work and $s^{\epsilon}$ the entropy. It is worth noticing that $\gamma^{\epsilon}$ comes from the part of free energy rate which is due exclusively to the internal variables:

$$
\dot{\psi}^{\epsilon}=\dot{\psi}^{\epsilon}\left(\boldsymbol{\varepsilon}^{\epsilon}, \theta^{\epsilon}, \boldsymbol{a}^{\epsilon}\right)=\frac{\partial \psi^{\epsilon}\left(\boldsymbol{\varepsilon}^{\epsilon}, \theta^{\epsilon}, \boldsymbol{a}^{\epsilon}\right)}{\partial \boldsymbol{\varepsilon}^{\epsilon}}: \dot{\boldsymbol{\varepsilon}}^{\epsilon}+\frac{\partial \psi^{\epsilon}\left(\boldsymbol{\varepsilon}^{\epsilon}, \theta^{\epsilon}, \boldsymbol{a}^{\epsilon}\right)}{\partial \theta^{\epsilon}} \dot{\theta}^{\epsilon}+\frac{\partial \psi^{\epsilon}\left(\boldsymbol{\varepsilon}^{\epsilon}, \theta^{\epsilon}, \boldsymbol{a}^{\epsilon}\right)}{\partial \boldsymbol{a}^{\epsilon}}: \dot{\boldsymbol{a}}^{\epsilon},
$$

from which, according to the classical thermodynamics,

$$
\dot{\psi} \epsilon\left(\boldsymbol{\varepsilon}^{\epsilon}, \theta^{\epsilon}, \boldsymbol{a}^{\epsilon}\right)=\boldsymbol{\sigma}^{\epsilon}: \dot{\boldsymbol{\varepsilon}}^{\epsilon}-s^{\epsilon} \dot{\theta}^{\epsilon}-\gamma^{\epsilon} .
$$

It is recalled that, in Sect. 5, in the context of generalized standard materials (GSM), the free energy was assumed to depend on a finite number of internal variables, at both the micro- and the macrolevel (see (82), in order to deduce that the overall behavior of generalized standard constituents is also GSM behavior. The key point was the variation of the free energy as a sum of a finite number of products.

Regarding (235) it is easily realized that only the mean value of the third term cannot take an explicit form of products of mean values, contrarily to the first term (Hill-Mandel lemma) and the second term (strong convergence of the temperature [38]). This is natural, since internal variables, that appear in (233), are not controlled by the boundary conditions.

The quasistatic plastic evolutions are treated by dividing the total time into intervals and satisfying the stability and energy conditions in every interval. The internal variables coexist in both the micro- and the macroscale and act as intermediate parameters in the micro-macrotransition. Their convergence $\left(L^{2}\right.$ or in measure) is shown in Sects. 3, 4 and 8 and the effective constitutive laws depend on the values of internal 
variables in the unit cell. The two-scale plastic flow follows the rule of normality to the $\boldsymbol{y}$-dependent yield surface. In the two-scale limit of the plastic evolution, this dependence cannot be integrated out. Thus, the total dissipation is given by a combination of micro- and macrovariables.

A complete study of incremental methods should comprise the return mapping algorithm [161,180,181], the adaptive finite element methods [76], the general algorithms for multiscale analyses [207], the computational micro-to-macro transitions of discretized microstructures by [135], the strain-driven homogenization based on incremental formulation [134] and the aforementioned fully implicit formulation [17]. This study, justified by the mathematical theory $[122,138,139]$, could be enriched by the modeling and engineering applications $[26,80,110]$. Of special interest for this review is the convex cutting plane technique, proposed by Simo and Hughes [180] and developed by Lagoudas and coworkers [80,173], which allows for an incremental, linearized formulation of the fully coupled thermomechanical problem, based on the incremental linear constitutive expression of stress $\boldsymbol{\sigma}$, heat flux $\boldsymbol{q}$ and energy equation source $r$, as well as of the internal variables, in terms of the macroscopic strain and the macroscopic temperature at both the micro- and macrolevel, where all corresponding tangent moduli are assumed constant during the time step. Then, the two problems (macroscale and cell problem) are solved simultaneously using an iterative scheme: from the macroscale analysis, the macroscopic strain, temperature, and temperature gradient are computed, which are used in the cell problem to compute the microscale variables and the unknown stress, heat flux, etc. Moreover, from the microproblem, additional information is provided for the computation of the macroscopic tangent moduli required for the macroscale analysis [38]. This technique allows for bypassing the internal variables and compute the incremental local dissipation in terms of macrostrain and macrotemperature at both scales.

\section{Conclusion}

In this paper we presented a review on a mathematical homogenization theory of dissipative composites and its applications to the numerical analysis of engineering structures. The theory is founded on an existence theory of the partial differential equations describing the heterogeneous problem, whose review was presented too. The paper covered the principal lines of homogenization procedure for both rate-dependent and rate-independent materials, revealing their connection and their differences.

The review focused on the role of dissipation of generalized standard materials and specifically to its two-scale nature coming from the part of free energy rate which is due to the internal variables. The interplay between homogenization, dissipation and adequate functional setting was presented through the recent progress on dissipative materials. Emphasis was given in the recent homogenization theory of perfectly elastoplastic evolutions. Papers on issues as the constitutive equations on discontinuity surfaces, or the uniqueness of elastic-perfectly plastic processes, or the criteria of shear banding in heterogeneous elastoplastic evolutions, were reviewed and related to the classical works in mechanics. Special attention was also given to works bridging the gap between numerical analysis and computational techniques, especially in the presence of strain discontinuities.

Of special interest was the mathematical theory of quasistatic energetic models that justify completely the use of incremental computational techniques, based on return mapping algorithm, tangent modulus and convex cutting plane techniques, which were critically surveyed.

Acknowledgements The authors wish to thank the anonymous reviewers for their constructive comments. The first author wishes to express his gratitude to the laboratory LEM3-UMR 7239 CNRS, Arts et Metiers ParisTech, Metz, for his worm hospitality during September-October 2016.

\section{References}

1. Aboudi, J.: Micromechanics-based thermoviscoelastic constitutive equations for rubber-like matrix composites at finite strains. Int. J. Solids Struct. 41, 5611-5629 (2004)

2. Aboudi, J., Pindera, M.-J., Arnold, S.M.: Higher-order theory for periodic multiphase materials with inelastic phases. Int. J. Plast 19(6), 805-847 (2003)

3. Alber, H.-D.: Materials with memory. In: Lecture Notes in Mathematics, vol. 1682. Springer-Verlag, Berlin (1998)

4. Allaire, G.: Homogenization and two-scale convergence. SIAM J. Math. Anal. 23, 1482-1518 (1992)

5. Allaire, G.: Shape Optimization by the Homogenization Method. Springer, New York (2002)

6. Allaire, G., Bonnetier, E., Francfort, G., Jouve, F.: Shape optimization by the homogenization method. Numer. Math. 76, 27-68 (1997) 
7. Allaire, G., Briane, M.: Multiscale convergence and reiterated homogenization. Proc. R. Soc. Edinb. A 126, 297-342 (1996)

8. Allaire, G., Brizzi, R.: A multiscale FEM for numerical homogenization. Multiscale Model. Simul. 4(3), 790-812 (2005)

9. Allaire, G., Kohn, R.V.: Explicit optimal bounds on the elastic energy of a two-phase composite in two space dimensions. Q. Appl. Math. 51(4), 675-699 (1993)

10. Allaire, G., Kohn, R.V.: Optimal bounds on the effective behavior of a mixture of two well-ordered elastic materials. Q. Appl. Math. 51(4), 643-674 (1993)

11. Allaire, G., Kohn, R.V.: Topology optimization and optimal shape design using homogenization. In: Topology Design of Structures, Springer, Dordrecht (1993)

12. Amar, M.: Two-scale convergence and homogenization in BD. Asymptot. Anal. 16, 65-84 (1998)

13. Anzellotti, G., Giaquinta, M.: Existence of the displacements field for an elasto-plastic body subject to Hencky's law and von Mises yield condition. Manuscr. Math. 32, 101-136 (1980)

14. Anzellotti, G., Luckhaus, S.: Dynamical evolution of elasto-plastic bodies. Appl. Math. Optim. 15(1), 121-140 (1987)

15. Armero, F., Garikipati, K.: Recent advances in the analysis and numerical simulation of strain localization in inelastic solids. In: Owen, D.R.J., Oñate, E. (eds.) Proceedings of COMPLAS IV, 4th international conference on computational plasticity, pp. 547-561. Pineridge Press, Swansea (1995)

16. Asada, T., Ohno, N.: Fully implicit formulation of elastoplastic homogenization problem for two-scale analysis. Int. J. Solids Struct. 44(22-23), 7261-7275 (2007)

17. Asada, T., Tanaka, Y., Ohno, N.: Two-scale analysis of honeycombs indented by flat punch. In: Huh, H., Park, C., Lee, C., Keum, Y. (eds) Engineering Plasticity and its Applications. From Nanoscale to Macroscale. Proceedings of AEPA 2008, pp. 819-824. World Scientific Publishing, Singapore (2009)

18. Babadjian, J.-F.: Méthodes variationnelles pour l'étude de milieux dissipatifs: applications en rupture, endommagement et plasticité. Université Pierre et Marie Curie, Habilitation (2013)

19. Benaarbia, A., Chrysochoos, A., Robert, G.: Thermomechanical behavior of PA6.6 composites subjected to low cycle fatigue. Compos. B 76, 52-64 (2015)

20. Brassart, L., Stainier, L.: On convergence properties of variational constitutive updates for elasto-visco-plasticity. GAMMMitteilungen 35(1), 26-42 (2012)

21. Brassart, L., Stainier, L., Doghri, I., Delannay, L.: Homogenization of elasto-(visco) plastic composites based on an incremental variational principle. Int. J. Plast 36, 86-112 (2012)

22. Bravo-Castillero, J., Rodríguez-Ramos, R., Mechkour, H., Otero, J.A., Cabanas, J.H., Sixto, L.M., Guinovart-Díaz, R., Sabina, F.J.: Homogenization and effective properties of periodic thermomagnetoelectroelastic composites. J. Mech. Mater. Struct. 4(5), 819-836 (2009)

23. Bridgman, P.W.: The thermodynamics of plastic deformation and generalized entropy. Rev. Mod. Phys. 22(1), 56-63 (1950)

24. Bruhns, O.: Some remarks on the history of plasticity. Heinrich Hencky, a pioneer of the early years. In: Stein, E. (ed.) The History of Theoretical, Material and Computational Mechanics-Mathematics Meets Mechanics and Engineering, vol. 1, Lecture Notes in Applied Mathematics and Mechanics, pp. 133-152. Springer, Berlin, Heidelberg (2014)

25. Carstensen, C., Hackl, K., Mielke, A.: Non-convex potentials and microstructures in finite-strain plasticity. Proc. R. Soc. A 458, 299-317 (2002)

26. Cavalcante, M.A.A., Khatam, H., Pindera, M.J.: Homogenization of elastic-plastic periodic materials by FVDAM and FEM approaches-an assessment. Compos. Part B Eng. 42, 1713-1730 (2011)

27. Cavalcante, M.A.A., Marques, S.P.C., Pindera, M.J.: Computational aspects of the parametric finite-volume theory for functionally graded materials. Comput. Mater. Sci. 44, 422-438 (2008)

28. Cavalcante, M.A.A., Marques, S.P.C., Pindera, M.J.: Transient thermomechanical analysis of a layered cylinder by the parametric finite-volume theory. J. Therm. Stresses 32, 112-134 (2009)

29. Cavalcante, M.A.A., Pindera, M.-J.: Finite-volume enabled transformation field analysis of periodic materials. Int. J. Mech. Mater. Des. 9, 153-179 (2013)

30. Cavalcante, M.A.A., Pindera, M.J.: Generalized FVDAM theory for elastic-plastic periodic materials. Int. J. Plast 77, 90-117 (2016)

31. Chaboche, J., Kanoute, P., Ross, A.: On the capabilities of mean field approaches for the description of plasticity in metal matrix composites. Int. J. Plast. 21, 1409-1434 (2005)

32. Chaboche, J., Kruch, S., Maire, J., Pottier, T.: Towards a micromechanics based inelastic and damage modeling of composites. Int. J. Plast. 17, 411-439 (2001)

33. Charalambakis, N., Murat, F.: Weak solutions to initial boundary value problems for the shearing of nonhomogeneous thermoviscoplastic materials. Proc. R. Soc. Edinb. 113A, 257-265 (1989)

34. Charalambakis, N., Murat, F.: Approximation by finite elements, existence and uniqueness for a model of stratified thermoviscoplastic materials. Ricerche Mat. 55, 171-218 (2006)

35. Charalambakis, N., Murat, F.: Homogenization of stratified thermoviscoplastic materials. Q. Appl. Math. 64, 359-399 (2006)

36. Charalambakis, N., Murat, F.: Stability by homogenization of thermoviscoplastic problems. Math. Models Methods Appl. Sci. 20, 1591-1616 (2010)

37. Charalambakis, N., Murat, F.: Two stable by homogenization models in simple shearing of rate-dependent non-homogeneous materials. Q. Appl. Math. 68, 395-419 (2010)

38. Chatzigeorgiou, G., Charalambakis, N., Chemisky, Y., Meraghni, F.: Periodic homogenization for fully coupled thermomechanical modeling of dissipative generalized standard materials. Int. J. Plast. 81, 18-39 (2016)

39. Chatzigeorgiou, G., Chemisky, Y., Meraghni, F.: Computational micro to macro transitions for shape memory alloy composites using periodic homogenization. Smart Mater. Struct. 24, 035009 (2015)

40. Chatzigeorgiou, G., Javili, A., Steinmann, P.: Unified magnetomechanical homogenization framework with application to magnetorheological elastomers. Math. Mech. Solids 19(2), 194-212 (2014)

41. Chatzigeorgiou, G., Meraghni, F., Javili, A.: Generalized interfacial energy and size effects in composites. J. Mech. Phys. Solids 106, 257-282 (2017) 
42. Chen, W.F., Han, D.J.: Plasticity for Structural Engineers. Springer, Berlin (1988)

43. Cherkaev, A., Kohn, R.: Topics in the Mathematical Modelling of Composite Materials. Birkhäuser, Boston (1997)

44. Christensen, R.M., Lo, K.H.: Solutions for effective shear properties in three phase sphere and cylinder models. J. Mech. Phys. Solids 27, 315-330 (1979)

45. Ciarlet, P.: Mathematical Elasticity: Volume I, Three dimensional Elasticity, Studies in Mathematics and its Applications. North-Holland Elsevier Science Pub., Amsterdam (1988)

46. Dal Maso, G.: Intoduction to $\Gamma$-convergence. In: Brezis, H. (ed.) Progress in Non-linear Differential Equations and their Application, vol. 8. Springer Science+Business Media, LLC, Boston (1993)

47. Dal Maso, G., DeSimone, A., Mora, M.G.: Quasistatic evolution problems for linearly elastic-perfectly plastic materials. Arch. Ration. Mech. Anal. 180, 237-291 (2006)

48. De Giorgi, E.: Sulla convergenza di alcune successioni di integrali del tipo dell'area. Rendiconti Matematici 8, 277-294 (1975)

49. De Giorgi, E.: G-operators and $\Gamma$-convergence. PWN Polish Scientific Publishers and North-Holland, Amsterdam (1984)

50. Debordes, O.: Dualité des théorèmes statique et cinématique dans la théorie de l'adaptation des milieux continus élastoplastiques. Comptes Rendus de l'Académie des Sciences de Paris 282(Serie A), 535-537 (1976)

51. Debordes, O., Nayrolles, B.: Sur la théorie et le calcul a l'adaptation des structures élastoplastiques. J. Méc. 15, 1-53 (1976)

52. Defranceschi, A.: An introduction to homogenization and G-convergence. School on Homogenization and G-Convergence, ICTP, Trieste, September 6-17 (1993)

53. Desrumaux, F., Meraghni, F., Benzeggagh, M.L.: Generalised Mori-Tanaka scheme to model anisotropic damage using numerical Eshelby tensor. J. Compos. Mater. 35(7), 603-624 (2001)

54. Dodd, B., Bai, Y.: Adiabatic Shear Localization. Elsevier, London (2012)

55. Doghri, I., Ouaar, A.: Homogenization of two-phase elasto-plastic composite materials and structures: study of tangent operators, cyclic plasticity and numerical algorithms. Int. J. Solids Struct. 40, 1681-1712 (2003)

56. Drucker, D.C.: Variational principles in the mathematical theory of plasticity. Technical Report Nonr 562(10), Division of Applied Mathematics, Brown University (1956)

57. Drucker, D.C.: A definition of stable inelastic material. Technical Report Nonr 562(20)/2, Division of Applied Mathematics, Brown University (1957)

58. Duvaut, G., Lions, J.-L.: Les inéquations en Mécanique et en Physique. Dunod, Paris (1972)

59. Dvorak, G.: Transformation field analysis of inelastic composite materials. Proc. R. Soc. Lond. A 437, 311-327 (1992)

60. Dvorak, G., Bahel-El-Din, Y., Wafa, A.: Implementation of the transformation field analysis for inelastic composite materials. Comput. Mech. 14, 201-228 (1994)

61. Ebobisse, F., Reddy, B.D.: Some mathematical problems in perfect plasticity. Comput. Methods Appl. Mech. Eng. 193, 5071-5094 (2004)

62. Ekeland, I., Temam, R.: Convex Analysis and Variational Problems. North-Holland, Amsterdam (1976)

63. Eshelby, J .D.: The determination of the elastic field of an ellipsoidal inclusion, and related problems. Proc. R. Soc. Lond. A 241(1226), 376-396 (1957)

64. Fenchel, W.: Convex Cones, Sets, and Functions. Princeton University Press, Princeton (1953)

65. Fish, J., Shek, K., Pandheeradi, M., Shephard, M.: Computational plasticity for composite structures based on mathematical homogenization: theory and practice. Comput. Methods Appl. Mech. Eng. 148, 53-73 (1997)

66. Francfort, G., Giacomini, A.: Small strain heterogeneous elasto-plasticity revisited. Commun. Pure Appl. Math. 65, 1185$1241(2012)$

67. Francfort, G., Giacomini, A.: Heterogeneous Elasto-plasticity. In: Seminar in Laboratory J.-L. Lions. University Paris 6 , Paris (2013)

68. Francfort, G., Giacomini, A.: On periodic homogenization in perfect elasto-plasticity. J. Eur. Math. Soc. 16, 409-461 (2014)

69. Francfort, G., Giacomini, A.: The role of a vanishing interfacial layer in perfect elasto-plasticity. Chin. Ann. Math. Ser. B 36B(5), 813-828 (2015)

70. Francfort, G., Suquet, P.: Homogenization and mechanical dissipation in thermoviscoelasticity. Arch. Ration. Mech. Anal. 96, 268-293 (1986)

71. Francfort, G.A., Giacomini, A., Marigo, J.J.: The taming of plastic slips in Von Mises elasto-plasticity. Interfaces Free Bound. 17(4), 497-516 (2015)

72. Francfort, G.A., Giacomini, A., Marigo, J.J.: A case study for uniqueness of elasto-plastic evolutions: the bi-axial test. J. Math. Pures. Appl. 105(2), 198-227 (2016)

73. Francfort, G.A., Murat, F.: Homogenization and optimal bounds in linear elasticity. Arch. Ration. Mech. Anal. 94(4), 307-334 (1986)

74. Germain, P.: Sur certaines définitions liées à l'énergie en mécanique des solides. Int. J. Eng. Sci. 20(2), 245-259 (1982)

75. Greenberg, H.J.: Complementary minimum principles for an elastic-plastic material. Q. Appl. Math. 7, 85 (1948)

76. Guedes, J.M., Kikuchi, N.: Preprocessing and posprocessing for materials based on the homogenization method with adaptive finite element methods. Comput. Methods Appl. Mech. Eng. 83, 143-198 (1990)

77. Gurtin, M.E., Fried, E., Anand, L.: The Mechanics and Thermodynamics of Continua. Cambridge University Press, Cambridge (2009)

78. Halphen, B.: Sur les discontinuités de vitesse en elastoplasticité. Comptes Rendus de l'Académie des Sciences de Paris, Ser. A-B 287(7), A569-A572 (1978)

79. Halphen, B., Nguyen, Q.S.: Sur les matériaux standards généralisés. J. Méc. 14(1), 39-63 (1975)

80. Hartl, D.J., Chatzigeorgiou, G., Lagoudas, D.C.: Three-dimensional modeling and numerical analysis of rate-dependent irrecoverable deformation in shape memory alloys. Int. J. Plast. 26(10), 1485-1507 (2010)

81. Hashin, Z.: Theory of mechanical behavior of heterogeneous media. Appl. Mech. Rev. 17, 1-9 (1963)

82. Hashin, Z.: Analysis of composite materials: a survey. J. Appl. Mech. 50, 481-505 (1983)

83. Hashin, Z., Rosen, B.W.: The elastic moduli of fiber-reinforced materials. J. Appl. Mech. 31, 223-232 (1964) 
84. Hashin, Z., Shtrikman, S.: A variational approach to the theory of the effective magnetic permeability of multiphase materials. J. Appl. Phys. 33, 3125-3131 (1962)

85. Hill, R.: A variational principle of maximum plastic work in classical plasticity. Q. J. Mech. Appl. Mech. 1, 18-28 (1948)

86. Hill, R.: The Mathematical Theory of Plasticity. Oxford University Press, New York (1950)

87. Hill, R.: On the problem of uniqueness in the theory of a rigid-plastic solid-1. J. Mech. Phys. Solids 4, $247-255$ (1956)

88. Hill, R.: Acceleration waves in solids. J. Mech. Phys. Solids 10, 1-16 (1963)

89. Hill, R.: Elastic properties of reinforced solids: some theoretical principles. J. Mech. Phys. Solids 11, 357-372 (1963)

90. Hill, R.: The essential structure of constitutive laws for metal composites and polycrystals. J. Mech. Phys. Solids 15(2), 79-95 (1967)

91. Hill, R.: On macroscopic effects of heterogeneity in elastoplastic media at finite strain. Math. Proc. Camb. Philos. Soc. 95, 481-494 (1984)

92. Hill, R., Rice, J.: Constitutive analysis of elastic-plastic crystals at arbitrary strain. J. Mech. Phys. Solids 20(6), 401-413 (1972)

93. Hollister, S.J., Kikuchi, N.: A comparison of homogenization and standard mechanics analyses for periodic porous composites. Comput. Mech. 10, 73-95 (1992)

94. Houi, P.M., Stroud, D.: Nonlinear susceptibilities of granular media. Phys. Rev. B 37, 8719-8724 (1988)

95. Javili, A., Chatzigeorgiou, G., Steinmann, P.: Computational homogenization in magneto-mechanics. Int. J. Solids Struct. 50, 4197-4216 (2013)

96. Jendli, Z., Meraghni, F., Fitoussi, J., Baptist, D.: Multi-scales modelling of dynamic behaviour for discontinuous fibre SMC composites. Compos. Sci. Technol. 69(1), 97-103 (2009)

97. Jirásek, M.: Objective modeling of strain localization. Revue Française de Génie Civil 6(6), 1119-1132 (2002)

98. Johnson, C.: Existence theorems for plasticity problems. J. Math. Pures Appl. 55, 431-444 (1976)

99. Johnson, C.: On finite element methods for plasticity problems. Numer. Math. 26, 79-84 (1976)

100. Johnson, C.: A mixed finite element method for plasticity problems with hardening. SIAM J. Numer. Anal. 14, 575-583 (1977)

101. Johnson, C.: On plasticity with hardening. J. Math. Anal. Appl. 62, 325-335 (1978)

102. Kalamkarov, A.L., Andrianov, I.V., Danishevs'kyy, V.V.: Asymptotic homogenization of composite materials and structures. Appl. Mech. Rev. 62, 030802 (2009)

103. Kestin, J., Rice, J.R.: Paradoxes in the application of thermodynamics to strained solids. In: Stuart, E.G., Gal-Or, B., Brainard, A.J. (eds.) A Critical Review of Thermodynamics, pp. 275-298. Mono Book Corp, Baltimore (1970)

104. Khon, R.V., Milton, G.W.: On bounding the effective conductivity of anisotropic composites. In: Ericksen, J.L., Kinderlehrer, D., Kohn, R., Lions, J.-L. (eds.) Homogenization and Effective Moduli of Materials and Media, pp. 97-125. Springer-Verlag, New York (1986)

105. Kohn, R.V., Milton, G.W.: Variational bounds on the effective moduli of anisotropic composites. J. Mech. Phys. Solids 36, 597-629 (1988)

106. Kohn, R.V., Temam, R.: Dual spaces of stresses and strains, with applications to Hencky plasticity. Appl. Math. Optim. 10, $1-35(1983)$

107. Koiter, W.: General theorems for elasto-plastic solids. In: Sneddon, I.N., Hill, R. (eds.) Progress in Solid Mechanics, vol. 1, pp. 164-221. North-Holland, Amsterdam (1960)

108. Kruch, S., Chaboche, J.L.: Multi-scale analysis in elasto-viscoplasticity coupled with damage. Int. J. Plast. 27, 2026-2039 (2011)

109. Lagoudas, D., Hartl, D., Chemisky, Y., Machado, L., Popov, P.: Constitutive model for the numerical analysis of phase transformation in polycrystalline shape memory alloys. Int. J. Plast. 32-33, 155-183 (2012)

110. Lagoudas, D.C.: Shape Memory Alloys: Modeling and Engineering Applications. Springer, New York (2008)

111. Lagoudas, D.C., Gavazzi, A.C., Nigam, H.: Elastoplastic behavior of metal matrix composittes based on incremental plasticity and the Mori-Tanaka averaging scheme. Comput. Mech. 8, 193-203 (1991)

112. Lahellec, N., Suquet, P.: Nonlinear composites: a linearization procedure, exact to second-order in contrast and for which the strain-energy and the affine formulations coincide. Comptes Rendus Mécanique 332, 693-700 (2004)

113. Lahellec, N., Suquet, P.: Effective behavior of linear viscoelastic composites: a time-integration approach. Int. J. Solids Struct. 44, 507-529 (2006)

114. Lahellec, N., Suquet, P.: On the effective behavior of nonlinear inelastic composites: I. Incremental variational principles. J. Mech. Phys. Solids 55(9), 1932-1963 (2007)

115. Lahellec, N., Suquet, P.: On the effective behavior of nonlinear inelastic composites: II. A second order procedure. J. Mech. Phys. Solids 55(9), 1964-1992 (2007)

116. Lee, C.Y., Yu, W.: Variational asymptotic modeling of composite beams with spanwise heterogeneity. Comput. Struct. 89, 1503-1511 (2011)

117. Love, B., Batra, R.C.: Determination of effective thermomechanical parameters of a mixture of two elastothermoviscoplastic constituents. Int. J. Plast. 22, 1026-1061 (2006)

118. Lukkassen, D., Nguetseng, G., Wall, P.: Two-scale convergence. Int. J. Pure Appl. Math. 2, 33-81 (2002)

119. Lurie, K., Cherkaev, A.: Exact estimates of conductivity of composites formed by two isotropically conducting media taken in prescribed proportion. Proc. R. Soc. Edinb. A 99, 71-87 (1984)

120. Lurie, K., Cherkaev, A.: Exact estimates of conductivity of a binary mixture of isotropic components. Proc. R. Soc. Edinb. A 104, 21-38 (1986)

121. Maghous, S., Creus, G.J.: Periodic homogenization in thermoviscoelasticity: case of multilayered media with ageing. Int. J. Solids Struct. 40, 851-870 (2003)

122. Mainik, A., Mielke, A.: Existence results for energetic models for rate-independent systems. Calc. Var. Partial. Differ. Equ. 22, 73-99 (2005) 
123. Matthies, H., Strang, G., Christiansen, E.: The saddle point of a differential programm in energy methods in FEA. In: Glowinski, R., Robin, E., Zienckiewicz, O.C. (eds.) Energy Methods in Finite Element Analysis. Wiley and Sons, New York (1979)

124. McPhedran, R.C., McKenzie, D.R.: The conductivity of lattices and spheres: 1 . The simple cubic lattices. Proc. R. Soc. Lond. A 362, 45-63 (1978)

125. Meraghni, F., Benzeggagh, M.L.: Micromechanical modelling of matrix degradation in randomly oriented discontinuousfibre composites. Compos. Sci. Technol. 55(2), 171-186 (1995)

126. Mercier, B.: Sur la théorie et l'analyse numérique de problèmes de plasticité. Ph.D. thesis, Université Pierre et Marie Curie (Paris VI) (1977)

127. Mercier, S., Molinari, A.: Homogenization of elastic-viscoplastic heterogeneous materials: self-consistent and Mori-Tanaka schemes. Int. J. Plast. 25, 1024-1048 (2009)

128. Mercier, S., Molinari, A., Berbenni, S., Berveiller, M.: Comparison of different homogenization approaches for elasticviscoplastic materials. Modell. Simul. Mater. Sci. Eng. 20(2), 024004 (2012)

129. Michel, J.C., Galvanetto, U., Suquet, P.: Constitutive relations involving internal variables based on a micromechanical analysis. In: Maugin, G., Drouot, R., Sidoroff, F. (eds.) Continuum Thermomechanics: The Art and Science of Modelling Material Behaviour, pp. 301-312. Kluwer Academic Publishers, New York (2000)

130. Michel, J.C., Moulinec, H., Suquet, P.: Effective properties of composite materials with periodic microstructure: a computational approach. Comput. Methods Appl. Mech. Eng. 172, 109-143 (1999)

131. Michel, J.C., Suquet, P.: Nonuniform transformation field analysis. Int. J. Solids Struct. 40, 6937-6955 (2003)

132. Michel, J.C., Suquet, P.: Computational analysis of nonlinear composites structures using the nonuniform transformation field analysis. Comput. Methods Appl. Mech. Eng. 193, 5477-5502 (2004)

133. Michel, J.C., Suquet, P.: Non-uniform transformation field analysis: a reduced model for multiscale non-linear problems in solid mechanics. In: Galvanetto, U., Aliabadi, F. (eds.) Multiscale Modelling in Solid Mechanics: Computational Approaches, pp. 159-206. Imperial College Press, Singapore (2010)

134. Miehe, C.: Strain-driven homogenization of inelastic microstructures and composites based on an incremental variational formulation. Int. J. Numer. Methods Eng. 55, 1285-1322 (2002)

135. Miehe, C., Koch, A.: Computational micro-to-macro transitions of discretized microstructures undergoing small strains. Arch. Appl. Mech. 72(4-5), 300-317 (2002)

136. Mielke, A.: Private Communication

137. Mielke, A.: Existence of minimizers in incremental elastoplasticity with finite strains. SIAM J. Math. Anal. 36, 384-404 (2004)

138. Mielke, A.: Evolution of rate-independent systems. In: Dafermos, C.M., Feireisl, E. (eds.) Handbook of Differential Equations: Evolutionary Equations, vol. 2, pp. 461-559. North-Holland, Amsterdam (2005)

139. Mielke, A., Timofte, A.: Two-scale homogenization for evolutionary variational inequalities via the energetic formulation. SIAM J. Math. Anal. 39, 642-668 (2007)

140. Miksis, M.J.: Effective dielectric constant of a nonlinear composite material. SIAM J. Appl. Math. 43, 1140-1155 (1983)

141. Milton, G.: Bounds on the electromagnetic, elastic, and other properties of two-component composites. Phys. Rev. Lett. 46, 542-545 (1981)

142. Milton, G.: The coherent potential approximation is a realizable effective medium scheme. Commun. Math. Phys. 99, 463-500 (1985)

143. Milton, G.: Modelling the properties of composites by laminates. In: Ericksen, J.L., Kinderlehrer, D., Kohn, R., Lions, J.-L. (eds.) Homogenization and effective moduli of materials and media, pp. 150-174. Springer-Verlag, New York (1986)

144. Milton, G.: On characterizing the set of possible effective tensors: the variational method and the translation method. Commun. Pure Appl. Math. 43, 63-125 (1990)

145. Moreau, J.J.: Application of convex analysis to the treatment of elastoplastic systems. In: Germain, P., Nayrolles, B. (eds.) Applications of Methods of Functional Analysis to Problems in Mechanics, pp. 56-89. Springer, Berlin (1976)

146. Mori, T., Tanaka, K.: Average stress in matrix and average elastic energy of materials with misfitting inclusions. Acta Metall. 21(5), 571-574 (1973)

147. Mura, T.: Micromechanics of Defects in Solids. In: Mechanics of elastic and inelastic solids. Second, Revised edition, Kluwer Academic Publishers, Dordrecht (1987)

148. Murat, F.: H-convergence. Séminaire d'analyse fonctionnelle et numérique de l'Université d'Alger. Multicopied (1977)

149. Murat, F.: Compacité par compensation. Annali della Scuola Normale Superiore di Pisa 5, $489-507$ (1978)

150. Murat, F., Tartar, L.: Calcul des Variations et Homogeneisation, Les methodes de l'homogénéisation: Théorie et Applications en Physique. In: Coll. Dir. Etudes et Recherches EDF, pp. 319-369. Eyrolles, Paris (1985)

151. Murat, F., Tartar, L.: H-convergence, in topics in the mathematical modelling of composite materials. In: Cherkaev, A., Kohn, R.V. (eds.) Progress in Nonlinear Differential Equations and Their Applications, vol. 31, pp. 21-43. Birkhäuser, Boston (1997)

152. Needleman, A., Tvergaard, V.: Analyses of plastic localization in metals. Appl. Mech. Rev. 45(3, part 2), S3-S18 (1992)

153. Neff, P., Pauly, D., Witsch, K.J.: Poincare meets Korn via Maxwell: extending Korn's first inequality to incompatible tensor fields. J. Differ. Equ. 258(4), 1267-1302 (2015)

154. Nemat-Nasser, S., Hori, M.: Micromechanics: Overall Properties of Heterogeneous Materials, 2nd edn. North-Holland, Amsterdam (1999)

155. Nesenenko, S., Neff, P.: Homogenization for dislocation based gradient viscoplasticity. J. Math. Anal. Appl. 425(1), 133-159 (2015)

156. Nguetseng, G.: A general convergence result for a functional related to the theory of homogenization. SIAM J. Math. Anal. 20, 608-623 (1989)

157. Nguyen, Q.S.: Mechanical modelling of anelasticity. Rev. Phys. Appl. 23, 325-330 (1988)

158. Oliver, J.: Continuum modeling of strong discontinuities in Solid Mechanics. In: Owen, D.R.J., Oñate, E. (eds.) Proceedings of COMPLAS IV, 4th International Conference on Computational Plasticity, pp. 455-479. Pineridge Press, Swansea (1995) 
159. Oliver, J.: Modeling strong discontinuities in solid mechanics via strain softening constitutive equations. Part 1: fundamentals. Int. J. Numer. Methods Eng. 39, 3575-3600 (1996)

160. Oliver, J., Simo, J.: Modeling strong discontinuities by means of strain softening constitutive equations. In: Computer Modelling of Concrete Structures, Proceedings of EURO-C 1994, pp. 363-372. Pineridge Press, Swansea, UK (1994)

161. Ortiz, M., Simo, J.C.: An analysis of a new class of integration algorithms for elastoplastic constitutive relations. Int. J. Numer. Methods Eng. 23, 353-366 (1986)

162. Pindera, M.J., Khatam, H., Drago, A.S., Bansal, Y.: Micromechanics of spatially uniform heterogeneous media: a critical review and emerging approaches. Compos. B Eng. 40(5), 349-378 (2009)

163. Ponte-Castañeda, P.: A new variational principle and its applications to nonlinear heterogeneous systems. SIAM J. Appl. Math. 52, 1321-1341 (1992)

164. Ponte-Castañeda, P.: Bounds and estimates for the properties of nonlinear heterogeneous systems. Philos. Trans. A 340, 532-566 (1992)

165. Ponte-Castañeda, P.: New variational principles in plasticity and their application to composite materials. J. Mech. Phys. Solids 40, 1757-1788 (1992)

166. Ponte-Castañeda, P.: Exact second order estimates for the effective mechanical properties of nonlinear composite materials. J. Mech. Phys. Solids 44, 827-862 (1996)

167. Ponte-Castañeda, P.: Second-order homogenization estimates for nonlinear composites incorporating field fluctuations. I-theory. J. Mech. Phys. Solids 50, 737-757 (2002)

168. Ponte-Castañeda, P., Suquet, P.: Nonlinear composites. Adv. Appl. Mech. 34, 171-302 (1998)

169. Ponte-Castañeda, P., Willis, J.R.: Variational second-order estimates for nonlinear composites. Proc. R. Soc. Lond. A 455, 1799-1811 (1999)

170. Ponte-Castañeda, P.: The effective mechanical properties of nonlinear isotropic composites. J. Mech. Phys. Solids 39, 45-71 (1991)

171. Prager, W., Hodge, P.: Theory of Perfectly Plastic Solids. Wiley, New York (1951)

172. Puglisi, G., Truskinovsky, L.: Thermodynamics of rate-independent plasticity. J. Mech. Phys. Solids 53, 655-679 (2005)

173. Qidwai, M.A., Lagoudas, D.C.: Numerical implementation of a shape memory alloy thermomechanical constitutive model using return mapping algorithms. Int. J. Numer. Methods Eng. 47, 1123-1168 (2000)

174. Qu, J., Cherkaoui, M.: Fundamentals of Micromechanics of Solids. Wiley, New Jersey (2006)

175. Schweizer, B., Veneroni, M.: Periodic homogenization of the Prandtl-Reuss model with hardening. J. Multiscale Model. 2, 69-106 (2010)

176. Sengupta, A., Papadopoulos, P., Taylor, R.L.: A multiscale finite element method for modeling fully coupled thermomechanical problems in solids. Int. J. Numer. Methods Eng. 91, 1386-1405 (2012)

177. Simo, J., Oliver, J.: A new approach to the analysis and simulation of strong discontinuities. In: Bažant, Z.P., Bittnar, Z., Jirásek, M., Mazars, J. (eds.) Fracture and Damage in Quasibrittle Structures, pp. 25-39. E\&FN Spon, London (1994)

178. Simo, J., Oliver, J., Armero, F.: An analysis of strong discontinuities induced by strain-softening in rate-independent inelastic solids. Comput. Mech. 12, 277-296 (1993)

179. Simo, J.C.: A new methodology for the numerical simulation of strain softening in inelastic solids. Technical Report CR 95.002, Naval Facilities Engineering Service Center, Port Hueneme, California (1994)

180. Simo, J.C., Hughes, T.J.R.: Computational Inelasticity. Springer-Verlag, New York (1998)

181. Simo, J.C., Taylor, R.T.: A return mapping algorithm for plane stress elastoplasticity. Int. J. Numer. Meth. Eng. 22, 649-670 (1986)

182. Solombrino, F.: Quasistatic evolution problems for nonhomogeneous elastic plastic materials. J. Convex Anal. 16, 89-119 (2009)

183. Solombrino, F.: Quasistatic evolution in perfect plasticity for general heterogeneous materials. Arch. Ration. Mech. Anal. 212(1), 283-330 (2014)

184. Spagnolo, S.: Convergence in energy for elliptic operators. In: Hubbart, B. (ed.) Numerical Solutions of Partial Differential Equations III, pp. 469-498. Academic Press, New York (1976)

185. Suquet, P.: Existence et régularité des solutions des équations de la plasticité. Comptes Rendus de l'Académie des Sciences de Paris 286(Serie A), 1201-1204 (1978)

186. Suquet, P.: Un espace fonctionnel pour les équations de la plasticité. Annales de la Faculté des Sciences de Toulouse, 5e série 1(1), 77-87 (1979)

187. Suquet, P.: Evolution problems for a class of dissipative materials. Q. Appl. Math. 38, 391-414 (1981)

188. Suquet, P.: Sur les équations de la plasticité: existence et régulatité des solutions. J. Méc. 20(1), 1-39 (1981)

189. Suquet, P.: Plasticité et homogénéisation. Ph.D. thesis, Université Pierre et Marie Curie (Paris VI) (1982)

190. Suquet, P.: Discontinuities and Plasticity. In: Panagiotopoulos, P., Moreau, J.-J. (eds.) Nonsmooth Mechanics and Applications, International Center for Mechanical Sciences, Courses and Lectures, vol. 302, pp. 280-340. Springer Vienna, Vienna (1988)

191. Suquet, P.: Overall properties of non-linear composites: a modified secant moduli theory and its link with Ponte Castañeda's nonlinear variational procedure. Comptes Rendus de l'Académie des Sciences de Paris, Serie IIb 320, 563-571 (1995)

192. Suquet, P.: Effective properties of nonlinear composites. In: Suquet, P. (ed.) Continuum Micromechanics, pp. $197-264$. Springer Vienna, Vienna (1997)

193. Suquet, P.M.: Elements of homogenization for inelastic solid mechanics. Lecture Notes in Physics, vol. 272, pp. $193-278$. Springer, Berlin (1987)

194. Talbot, D.R.S., Willis, J.R.: Variational principles for inhomogeneous non-linear media. IMA J. Appl. Math. 35, 39-54 (1985)

195. Talbot, D.R.S., Willis, J.R.: Bounds and self-consistent estimates for the overall properties of nonlinear composites. IMA J. Appl. Math. 39, 215-240 (1987)

196. Tartar, L.: Homogénéisation et compacité par compensation. Cours Peccot, Collège de France (1977)

197. Tartar, L.: Nonlinear Constitutive Relations and Homogenization. North-Holand, Amsterdam (1978) 
198. Tartar, L.: Etude des oscillations dans les equations aux derivees partielles non-lineaires. Lect. Notes Phys. 195, 385-412 (1984)

199. Tartar, L.: Estimations fines de coefficients homogénéisés. In: Kree, P. (ed.) Ennio De Giorgi Colloquium (Research Notes in Mathematics), pp. 168-187. Pitman, Boston (1985)

200. Tartar, L.: Memory effects and homogenization. Arch. Ration. Mech. Anal. 111, 121-133 (1990)

201. Tartar, L.: General Theory of Homogenization. A Personalized Introduction. In: Lecture Notes of the Unione Matematica Italiana, vol. 7. Springer, Berlin (2009)

202. Temam, R.: Existence theorems for the variational problems of plasticity. In: Atteia, M., Bancel, D., Gumowski, I. (eds.) Nonlinear Problems of Analysis in Geometry and Mechanics, pp. 57-70. Pitman, London (1981)

203. Temam, R.: On the continuity of the trace of vector functions with bounded deformation. Appl. Anal. Int. J. 11(4), 291-302 (1981)

204. Temam, R.: Approximation de fonctions convexes sur un espace de mesures et applications. Can. Math. Bull. 25, 392-413 (1982)

205. Temam, R., Strang, G.: Duality and relaxation in the variational problems of plasticity. J. Méc. 19, 493-527 (1980)

206. Temam, R., Strang, G.: Functions of bounded deformation. Arch. Ration. Mech. Anal. 75, 7-21 (1980)

207. Terada, K., Kikuchi, N.: A class of general algorithms for multi-scale analyses of heterogeneous media. Comput. Methods Appl. Mech. Eng. 190, 5427-5464 (2001)

208. Tsalis, D., Baxevanis, T., Chatzigeorgiou, G., Charalambakis, N.: Homogenization of elastoplastic composites with generalized periodicity in the microstructure. Int. J. Plast. 51, 161-187 (2013)

209. Tsalis, D., Chatzigeorgiou, G., Tsakmakis, C., Charalambakis, N.: Dissipation inequality-based periodic homogenization of wavy materials. Compos. B 76, 89-104 (2015)

210. Tu, W., Pindera, M.J.: Targeting the finite-deformation response of wavy biological tissues with bio-inspired material architectures. J. Mech. Behav. Biomed. Mater. 28, 291-308 (2013)

211. Tzavaras, A.: Effect of thermal softening in shearing of strain-rate dependent materials. Arch. Ration. Mech. Anal. 99(4), 349-374 (1987)

212. Visintin, A.: Homogenization of the nonlinear Kelvin-Voigt model of viscoelasticity and of the Prager model of plasticity. Continuum Mech. Thermodyn. 18, 223-252 (2006)

213. Visintin, A.: Homogenization of the nonlinear Maxwell model of viscoelasticity and of the Prandtl-Reuss model of elastoplasticity. Proc. R. Soc. Edinb. 138 A, 1363-1401 (2008)

214. Walpole, L.J.: On bounds for the overall elastic moduli of inhomogeneous systems-I. J. Mech. Phys. Solids 14, 151-162 (1966)

215. Walpole, L.J.: On the overall elastic moduli of composite materials. J. Mech. Phys. Solids 17, 235-251 (1969)

216. Willis, J.: Bounds and self-consistent estimates for the overall properties of anisotropic composites. J. Mech. Phys. Solids 25, 185-202 (1977)

217. Willis, J.R.: Variational estimates for the overall response of an inhomogeneous nonlinear dielectric. In: Ericksen, J.L., Kinderlehrer, D., Kohn, R., Lions, J.-L. (eds.) Homogenization and Effective Moduli of Materials and Media, pp. 247-263. Springer-Verlag, New York (1986)

218. Wright, T.W.: The Physics and Mathematics of Shear Bands. Cambridge University Press, Cambridge (2002)

219. Zeng, X.C., Bergman, D.J., Houi, P.M., Stroud, D.: Effective medium theory for weakly nonlinear composites. Phys. Rev. B 38, 10970-10973 (1988)

220. Zhigang, S., Chaoxian, Z., Xiguang, G., Yingdong, S.: A quadrilateral element-based method for calculation of multi-scale temperature field. Chin. J. Aeronaut. 23, 529-536 (2010) 